\title{
Scientific Papers
}

\section{[1]}

\section{Choosing an Infertility Center in the United States of America: An Internet Based Approach}

\author{
Ahmed Mohamed Abou-Setta, Ragaa Mansour, Gamal Serour and Mohamed Aboulghar \\ The Egyptian IVF-ET Center, Hadayek El-Maadi, Egypt
}

Background: Even though approximately 6 million American women become pregnant each year, giving birth to more than 10,000 newborns each day, the latest national survey has revealed that $7 \%$ of married couples, in which the woman was of reproductive age (2.1 million couples), reported that they had not used contraception for 12 months and still the woman had not become pregnant [1,2]. At the turn of the century, there were 408 fertility centers operating in the United States alone [2]. These centers differ in size, geographic distribution and success rates. Therefore choosing which one is most appropriate for a couple can be difficult. In addition, there is an uneven distribution of infertility centers over the individual states. Furthermore, this uneven allocation is not only at the state level, with three states not containing any IVF centers, but also on the metropolitan level, where some cities contain more centers that their geographical and population share. In light of these facts, and in the scope that the Internet has become the newest and fastest growing marketplace for medical, and/or medical-related, businesses, more and more patients are going to the Internet in search of treatment plans for infertility, and/or an infertility center $[3,4]$. Health related web sites are now amongst the most frequently accessed sites on the internet with current estimates indicating that there are now over 100,000 sites offering health related information [5]. Moreover, since IVF procedures are costly and most insurance and managed care programs do not completely, or sometimes even partially, cover all expenses, couples are more willing to travel for more efficient and cost-effective treatment [6].

Conclusions: A detailed analysis is underway, but a preliminary analysis shows that generally there is an adherence to FTC and SART rules and regulations. In contrast, ethical codes are enforced on a voluntary case-by-case scenario, with the majority showing some degree of adherence, and a minority showing strict adherence to one of the codes of ethics tested. The final conclusion will be presented. Finally an overall evaluation of infertility center websites will be given, in addition to recommendations to infertile couples seeking information on infertility centers via the Internet.

\section{References}

[1] Safe Motherhood: Promoting Health for Women Before, During, and After Pregnancy 2003. Department of Health and Human Services, Centers for Disease Control and Prevention. http://www.cdc.gov/nccdphp/aag/aag_drh.htm.

[2] 2000 Assisted Reproductive Technology Success Rates: National Summary and Fertility Clinic Reports. Department of Health and Human Services, Centers for Disease Control and Prevention. http://apps.nccd.cdc.gov/art00/clinics00acc.asp.

[3] A. Lass and P. Brinsden, How do patients choose private in vitro fertilization treatment? A customer survey in a tertiary fertility center in the United Kingdom, Fertil Steril 75(5) (May 2001), 893-897.

[4] A. Weissman, L. Gotlieb, S. Ward, E. Greenblatt and R.F. Casper, Use of the internet by infertile couples, Fertil Steril 73(6) (June 2000), 1179-1182.

[5] G. Eysenbach, E.R. Sa and T.L. Diepgen, Shopping around the internet today and tomorrow: towards the millennium of cybermedicine, British Medical Journal 319 (1999), 1294. 
[6] R.E. Blackwell, K.R. Hammond and M.P. Steinkampf, A one-year experience with a capitated health care plan for infertility, Fertil Steril 75(4) (April 2001), 749-753.

[2]

\section{Misinformation on the NET: Using Hereditary Hemochromatosis as a Model}

Randy Steven Alexander

Iron Disorders Institute, Greenville, USA

The Internet offers the most unique and powerful breakthrough in communication since the creation of Morse code. Daily, new users of all ages and ethnicity log on for the first time and witness the incredible power of this remarkable resource. They trace their genealogy through family history databases, order books, computers, almost anything imaginable but more importantly they search for information about their health and this is both hopeful and disturbing. As with anything, but especially this unique tool called the Internet, the possibility to cause harm is ever present. Harm comes in the form of so-called experts, who post medically or scientifically unsubstantiated health information to the NET. Patients do not always know how to distinguish what is valid and reliable and what is junk information. This is very much the case with a little known but very common genetic disorder called hereditary hemochromatosis. For the past decade individuals, universities and advocacy organions have been posting information about this condition to the NET. Some of what is posted is accurate, helpful and augments the patient-physician relationship.

The rest of it is doing great harm! Misinformation within the context of hemochromatosis can be devastating in the hands of an unknowing patient. This person can be driven to get inappropriately genetically tested and to have spouses and children also unnecessarily genetically tested. In the US especially, these individuals can lose their jobs and insurance as a result of the genetic findings, and yet the genetic test is not even needed to find out if hemochromatosis is present. To make matters worse, some of the hemochromatosis misinformation encourages patients to circumvent their physician and seek diagnosis and treatment through non-medical and often questionable means. This is not only reckless but potentially fatal for a patient who tries to treat hemochromatosis with unconventional and inappropriate methods. To illustrate how hemochromatosis could serve as a model used to address this problem on the Internet, a brief explanation of the condition will be helpful. Hemochromatosis (HHC) is an inherited disorder of iron metabolism and a leading cause of iron overload disease.

Individuals with hemochromatosis absorb more iron from the diet than do those with normal iron metabolism. Iron has no physiological means of excretion except blood loss during menstruation or pregnancy, so over time, the excess iron builds up in vital organs causing them to fail. People with undetected iron overload can die of a heart attack or develop liver cancer; they also can experience other forms of cancer, diabetes, arthritis, depression, infertility or impotence as a result of the excess iron. Hemochromatosis is real: the gene for this disorder, named HFE was discovered in 1996. Hemochromatosis is common; homozygous frequency is an average of 1:250 Caucasians depending upon origin. Among the Scots-Irish population 1:80 has the condition. Hemochromatosis can kill: if not detected and treated, a male can die of a heart attack in his late fifties; a female can also die of a heart attack 15-20 years after her period stops. Detection of iron overload requires simple blood work: fasting serum iron, TIBC and serum ferritin. The results can determine the treatment course. The treatment for hemochromatosis, when iron overload is present, is blood removal with therapeutic phlebotomy. As red blood cells are removed, the body is stimulated to make new red blood cells. Iron is pulled from ferritin, 
a containment protein present in every tissue of the body. As iron is pulled out of ferritin to make the new red blood cells, the danger of iron overload decreases.

Why are these facts germane to the issue of misinformation on the NET? Because the following erroneous information about hemochromatosis is presently readily available on the NET: Hemochromatosis is a blood disease. Wrong: Hemochromatosis is a metabolic disorder People with hemochromatosis have lots of iron in their blood. Wrong: A unit of blood from an HHC patient contains no more iron than any other blood donor. The iron in HHC patients is contained in ferritin. You must get genetically tested to find out that you have hemochromatosis. Wrong: though the genetic test can tell a person whether or not they have the common mutations of the HFE gene, the genetic test tells them nothing about their iron levels, which is the real concern. If you take IP6 you don't need phlebotomies. Wrong, though a person might slow down the amount of iron being absorbed, nothing except specially formulated pharmaceuticals or blood loss can remove iron from the body.

These are only a few of the misleading statements that are currently on the Internet and patients are being hurt by this and much more information that is in many instances complex. No doubt other health conditions are being improperly represented on the NET as well. The physician patient relationship is degraded when an emotional patient insists that the physician follow some procedure or therapy found on the NET placed there by a person not qualified to counsel. Or worse, the patient disregards the advice of the physician opting for the "easy way out" promised by some unknown "expert". The Internet is a marvelous tool for learning, but management of the information that appears on the NET is uploaded in "Wild West" fashion. HONcode is a good way to identify reputable sites and being an HONcode member is a good start on helping patients and practicing physicians who are not up to date with this example must be able to identify sites with content they can trust as reliable. HONcode might consider appointing monitors among its members to evaluate sites according to accuracy and reliability (information becomes outdated) of content. Iron Disorders Institute knows the reputable sites that provide patient information about iron and would appreciate the responsibility of rating such sites.

\section{[3]}

New trends in Medical Care: Travel for Treatment and the Medical Tourist The emergence of Thailand as an international center for treatment Future shift: will governments start building medical facilities abroad?

Bill Andersen and Duane Patrick Lennie

THAIMMO - Thai Medical Marketing Organization, Bangkok, Thailand

Part One - The Concept behind the Growth of Travel for Treatment

For many years America and European countries imported software programmers from countries with excellent education but poor wages. India and Russia were leading suppliers of workers who were physically relocated. Now the pendulum has swung back, and US and European countries are sending their software requirements to Russi and India for completion. Many leading programmers have returned to their homelands and created firms providing excellence work at attractive prices. This is not a new phenomenon. Japanese engineers returned home from the USA and other countries to build a technology and manufacturing industry that rocked the world. The same global mechanics can be seen in the advent of a similar dynamic in international health treatment. For many years Europe and the USA welcomed doctors and specialists from countries around the world who were grateful for the opportunity 
to live, work and learn in wealthy countries. Many are returning home and developing the local medical infructure to compare with or exceed the western institutions where they worked.

\section{Part Two - Thailand's Development as a Major Provider of Medical Services to Travelling Patients}

The presentation will outline the following aspects of medical treatment in Thailand and how they have developed, including specific examples with facts, figures and slides: - QUALITY - Thailand has a large base of first rate medical practgitioners, including several world leaders. Many of these have returned from long careers in the USA and other countries to lead the development of medical excellence (with examples of specific doctors and the local centers they lead). - HIGH STANDARDS - A willingness to invest has seen the creation of medical facilities that compare favorably with those in western countries, which are managed according to international ISO standards (with examples of hospitals and medical centers). - UP TO DATE TECHNOLOGY - The will to provide the best treatment possible means that new treatments and technology are studied, implemented and available long before they become available in other countries (with specific examples). - SERVICE - The cheerful and patient nature of Thai people combine with relatively low wages to provide patient care levels far higher than in most other countries. Foreign patients remark that Thai hospitals 'feel like 4 and 5 star hotels but with better service'. Patient to staff ratios are far higher than in equivalent western institutions (with facts and figures to support this). - AVAILABILITY - Medical facilities are large and well-staffed so there are few waiting lists. Major surgeries such as bypass operations can normally be scheduled within 48 hours (with specific examples). - LOW COST - Due to financial devaluation, the cost of medical services is very low compared to that in most western countries. Costs are typically 50 percent or more below European prices, and even lower when compared to north american medical costs (with specific examples). These factors have led to a rapidly growing medical sector in Thailand that is focussed on providing service to patients from around the world. Of the thirty Thai medical institutions that focus on international patients, the largest hospital called Bumrungrad in Bangkok received more than 300,000 foreign patients last year, up from 40,000 in 1999 and the number is increasing by more than $10 \%$ per year.

\section{Part Three - The Role of the Internet}

The growth of the Thai medical industry catering to medical tourists or travelling patients has been internet-led. The Internet has created the ideal communication vehicle for prospective patients to make contact with hospitals, view their facilities, learn about treatments offered and more. Both patients and their doctors use e-mail to communicate directly with doctors and surgeons in Thailand prior to the visits, and the treatments are arranged and prices set via cyberspace. It is clear that the Internet will increasingly play the role of information-supplier to patients, providing them with a wide choice of suppliers in many countries and a level of choice unprecedented in human history. The websites used by medical service providers for this purpose are evolving rapidly in response to user requirements (examples will be shown).

Part Four - The future of Medical Tourism - where will it lead?

Medical tourism in Thailand has until now been driven by individual patients discovering that they can obtain excellent medical care with very high levels of service at very attractive cost. It has also been driven by patients requiring treatments for which the cost is too high or the waiting list is too long in their own country. A new trend is that retired people are moving from European and Western countries 
to live in Thailand where they can enjoy the climate, and also low health care costs. In one retirement village in southern Thailand it is possible for elderly residents to have in-home care staff who work under the supervision of a resident doctor for just 300 Euros per month. Clearly governments face the same problems. With life expectancy increasing governments are facing the seemingly irreconcilable problem of caring for larger populations with a smaller percentage of taxpayers while costs rocket. I believe that medical establishments and governments will soon follow their citizens to new destinations. Already a major Swedish healthcare company is building a recuperation center near Bangkok. A recent Dutch study investigated the feasibility of building a large mental heath care facility in southern Thailand. A senior European health official visited some of our hospitals in Bangkok recently and was amazed by the high quality of treatment and care and the low cost. Responsible for both medical insurance and hospitals in a large area, he quipped about contracting to send thousands of patients a year from German to Bangkok for treatment, a solution he said would enable him to close on hospital and possibly meet his operating budgets for next year.

\title{
Conclusion
}

I believe that the increase in medical tourism offers a clear indication of what lies in the future. As western countries are faced with more elderly and other patients and rocketing medical costs they will start to move patients to other countries where it is possible to provide good medical care at a fraction of the cost. This may initially be offered as an optional extra: - Mr Jones, you need bypass surgery. Local surgery is available but there is an eight month waiting list. If you and your wife with to fly to Bangkok for treatment and remain in Thailand for a few weeks rest on the beach, we can arrange surgery for next week. - Mr and Mrs Johanssen, we know you are not happy with this old age home. Would you prefer to spend 6 months or more in Thailand, where we now operate a new facility. It is near the beach with warm climate and as you can see form the brochures is more like a three or four star hotel. Dont be surpised one day when your doctor tells you that the major treatment you require for surgery, hip or knee replacement or more comes with an airline ticket!

\section{[4]}

\section{Management conflict of interest in medical education and information: The Edott Portal}

\author{
Chiara Andreoli ${ }^{\mathrm{a}}$, Nunzio Guido Mangano ${ }^{\mathrm{b}}$ and Nadia Nicolis ${ }^{\mathrm{a}}$ \\ ${ }^{\mathrm{a}}$ GlaxoSmithKline, Verona, Italy \\ ${ }^{\mathrm{b}}$ Dematel srl, Italy
}

Today, the role a pharmaceutical company is expected to play is no longer merely limited to the research and marketing of drugs, but increasingly involved in the offer and supply of forward-thinking healthcare services, and especially in the e-learning or ECM sector. Consequently, the key feature is the quality of the information and instruction provided. Obviously, the information communicated by a company must be in tune with the current trends in medicine, based on scientific tests rather than on opinions, and correct learning models must be guaranteed two conditions that are essential and beyond dispute. What we really want to emphasize is the importance of openly facing up to the problems concerning the independence of the providers of information and learning as well as conflict of interests already present in the medical world. A preliminary market research conducted in Italy on GPs revealed that there was a strong unfulfilled need in the medical community, and particularly among GPs, for a specific healthcare site offering medical information and learning on line (CME) in Italian, as well as a 
tool allowing dialogue and exchange views among colleagues. So, in 2000 Glaxosmithkline decided to set up the eDott portal, to provide a useful service for the professional preparation of family doctors in Italy.

The name of the company sponsoring the site is clearly indicated on the homepage, but eDott.it is not the communication channel chosen by GlaxoSmithKline to publicize and promote its products: the aims, the structure and the methods are clearly distinct from those of the official GSK.it site. The portal contents have in fact been focused on the following key areas:

- INFORMATION: free access to international databases (i.e. free, full access to the Cochrane library) and information resources selected and filtered for the specific needs of GPs.

- SERVICES: useful tools for GPs in their daily activity, i.e. customized bibliography search service, ask the expert service, etc.

- INTERACTIVE SERVICES AND COMMUNITY TOOLS: forum, clinical cases and questions.

- ACCREDITED CME AND DISTANCE LEARNING: publication of material on line as a support and integral part of official residential events.

Despite the features of eDott which clearly distinguish it from the official GSK site, the handling of the conflict of interests requires a thorough discussion of the related problems and the specific measures adopted to minimize any distortions which may occur. This is even more important in Italy, where the problem of independence and conflict of interests is a very delicate matter. The real problem is that these interests (economic, academic, etc.) have an ever increasing impact on political and social policies. Thus in Italy, as elsewhere, in medicine and increasingly in the health sector, the independence of research, publication of results, and instruction can no longer be taken for granted but must be clearly demonstrated. A conflict of interests exists and it is very pervasive, it cannot be denied nor should it be demonised, but it must be openly discussed, publicly disclosed and monitored.

The difference between Italy and other countries, especially those with an Anglo-Saxon culture and language, is not the prevalence and impact of the phenomenon itself, but rather the determination to ignore the problem and the absence of an active public counterpart providing independent information, to complement and balance the pressure on the part of sponsorized initiatives. So the editorial independence of the eDott portal with respect to GSK cannot be taken for granted, it must be the subject of an open debate which involves taking a clear stand. Although the eDott is economically dependent on its Sponsor and Founder, it is independent as regards its strategies, decisions and contents. Its credentials are mainly based on: The presence of an authoritative Technical Scientific Committee (CTS). The CTS was appointed to vouch for the editorial and scientific independence and provide guide lines for The future developments by constantly monitoring to ensure impartiality. quality of the products and services offered. The healthcare information available on the site is supplied by experts in the field of medicine with no advertising purposes whatsoever. Indeed, the site solemnly undertakes not to host any promotional messages or publicity concerning GSK drugs or those of The desire to provide the opportunity for formal occasions other companies. for instruction, under the auspices of the Ministry of Health. As regards ECM, the Sponsor finances the learning events and openly collaborates with the providers public or private bodies dedicated to instruction - for the organisation of these events as well as for the continuous monitoring of any conflict of interests which may arise. EDott, which since July 2001 is a real news channel, has obtained the HON certificate for the clear and reliable disclosure of the information provided. We believe that in situations such as that of Edott, with a multidisciplinary approach and the involvement of many different bodies (sponsors, contributors, users, accrediting bodies, etc.), a genuine vocation for quality instruction is not sufficient to dispel the fears of possible conflict of interests. 
Thus we are convinced of the importance of fully disclosing and continuously discussing the problem with a view to identifying and adopting any measures aimed at enhancing credibility. Furthermore, the discussion must not be a cosmetic operation designed to create a neutral facade of respectability: having discussed the problem it ceases to exist. The debate, stimulating the direct involvement of the users and, if necessary, all those interested at a public and private level, must lead to a clear standpoint and the adoption of measures enabling the user to navigate confidently within Edott. Regardless of the difficulties, an attempt must be made to establish and apply basic rules concerning the credentials of the guarantors, lecturers/pharmaceutical representatives and sponsors to avoid clashes between the legitimate interests of the instructors and industry, on the one hand, and patients and citizens on the other. It is, therefore, important to promote the number of occasions where the topic is discussed, to increase awareness and, through a formal disclosure of interest, declare all the links and economic and non-economic interests of every person involved, from the guarantor, to the contributor of information and the instructor.

[5]

\section{Using the Internet for Asthma Monitoring and Self-Management}

Jacob Anhoj, Anders Damgaard and Jacob Lenau AstraZeneca A/S, Albertslund, Denmark

A recent Cochrane review concluded that training in self-management involving self-monitoring appears to improve health outcomes for adults with asthma. Programmes which enables patients to adjust their medication according to a written action plan appear to be more effective than other forms of self-management (Gibson et al.: Cochrane Database Syst Rev 2000;(2):CD001117). Using the Internet for monitoring and self-management of chronic diseases (e.g. asthma, diabetes) has been proposed, and a small number of reports from pilot studies have been published (Finkelstein et al.: Chest. 2000 Jan;117(1):148-155). To our knowledge LinkMedica (www.linkmedica.dk) is the first widely available Internet based service enabling asthma patients to monitor their asthma and share data with their doctor or nurse via the Internet. LinkMedica was first launched in Denmark in May 2000. In late spring 2001 linkmedica.com and linkmedica.co.uk was launched. As of February 2003 the Danish site has nearly 8000 registered users and 1200 users have granted their doctor access to their asthma diary. System Description: In LinkMedicas asthma diary the patient enters peak flow and record symptoms daily. LinkMedica stores the data, calculates the level of asthma control and gives immediate feedback telling the patient what to do if his or her asthma is not well-controlled. When the patient and the doctor meet they can review changes in the patients asthma over time on the computer. LinkMedica can send reminders as e-mails or SMS messages if the patient forgets to fill in the diary before a specified time of the day. The algorithms used to calculate asthma control differ somewhat in the Danish site from the International site reflecting the fact that LinkMedica is prepared for local guidelines and traditions. Furthermore the Danish site contains a tool which the doctor may use as guidance when titrating the patients dose of inhaled steroid. When a doctor logs on to LinkMedica a patient list appears. A color (green, yellow, red) indicates the level of asthma control for each patient, provided the patient has entered data in the diary within the last three days. The questions and the algorithms controlling feed-back messages in the asthma diary have been designed and specified by independent, local advisory boards in UK and Denmark. Besides the asthma monitoring tool, LinkMedica contains a large number of articles summarizing scientific papers, Cochrane reviews, news and opinions regarding asthma and allergies. This content is selected by independent experts and approved by the advisory board. All content is revised and supplemented on 
a regular basis. Preliminary data from a controlled clinical study suggest that patients using LinkMedica have better lung function, less bronchial hyper-reactivity and lower asthma severity class as defined by GINA guidelines compared to patients treated by a specialist or a general practitioner (Rasmussen et al.: ATS 2003). In a recent user survey, semi-structured interviews revealed that patients and doctors appraise the diary in general. Patients experience improved asthma control when using it, and doctors find that the condition of patients using LinkMedica improves. However, patients have trouble pertaining to the diary for longer periods and usually stop using it after a short time unless they are coached by an enthusiastic doctor. Doctors, on the other hand, are reluctant to introduce LinkMedica to patients mainly due to time constraints in the clinic. These observations are supported by site statistics showing a steady increase in the number of registered users while the number of diary users is decreasing. Conclusions: LinkMedica is an Internet based service linking asthma patients and doctors. The core element of LinkMedica is the asthma diary enabling patients to monitor their asthma closely and alerting the patient in case the asthma is beginning to deteriorate. LinkMedica has been prepared for local setup in different countries allowing a local advisory board to customize the diary. Preliminary data from a controlled clinical study are encouraging suggesting improved asthma outcomes in patients using LinkMedica compared to patients being treated traditionally by lung specialists or general practitioners. However, despite positive feedback from users, it seems difficult to maintain diary users for more than short periods.

\section{[6]}

\section{Usability of Online Health Information for People with Disabilities}

Richard James Appleyard

Oregon Health \& Science University, Portland, USA

Current issues around the usability of online health information for people with disabilities are to be presented and discussed. This includes a review of the current literature relevant to this area, a review of the current methodology for assessing Web usability, and a proposed model for addressing these issues to increase the access to online health information by people with disabilities, in particular for those with cognitive impairments. Available preliminary data will also be presented. There are now estimated to be 49.7 million people with some form of disability in the US [1]. Of these, 14 million are aged 65 and over (equivalent to $42 \%$ of this age group). There is also the potential for the disabled population to grow significantly as the baby boom population continues to age. A digital divide exists with only $25 \%$ of persons with disabilities owning a computer and only $10 \%$ have Internet access. People with disabilities are less likely than people without disabilities to be online (43\% v. 57\%), and are less likely to be online from work (16\% v. 30\%) because far fewer are working [2]. Paradoxically, the Internet is having a much more positive impact on the lives of adults with disabilities who can access the Internet than those without disabilities. A number of publications [1,3] also suggest that people with disabilities are more likely to have health issues (early death, chronic conditions, preventable secondary conditions) and it is therefore more critical to identify and address barriers to access to health information that impact this group. For these reasons we have chosen to focus on eHealth Web sites in our studies. Since the arrival of the Web in 1994, there has been an increasing amount of attention to Web accessibility (for people with disabilities) [4] as well as Web usability by the general population [5]. However, there is a paucity of published usability studies evaluating Web sites by observing individuals with disabilities. The Disability Informatics Group (DIG) at the Oregon Institute on Disability \& Development (OIDD) is studying the usability of Web sites by people across a range of disability areas, i.e., sensory, mobility and cognitive. Web usability goes beyond the simpler concept of Web accessibility. Accessibility simply 
determines whether a site is navigable by individuals with restrictions or limitations on their interface with the computer. Usability focuses on the ability of the user to perform the functions and tasks facilitated on the site. Most Web Accessibility initiatives and guidelines have focused their requirements and recommendations in the area of sensory disabilities, i.e., users with vision and hearing impairments. But there is also a need for improved understanding of the difficulties individuals with cognitive disabilities encounter when attempting to use the Internet. Individuals with cognitive disabilities have difficulty using the Internet even after they have gained physical access. Internet access is important because it provides resources and information, as well as opportunities for socialization and support. There have been few published research studies identifying and assessing the Web usability issues for this population and how to remediate them. Our initial studies employ contextual interviews, field observations, user questionnaires and focus groups of individuals with cognitive disabilities, and their direct support or caregivers. The goal is to discern the online barriers encountered, the reasons for accessing or not accessing online services, and the possible misperceptions about the benefits and caveats of the Internet. This information will be used to guide later studies to investigate the usability of a selection of eHealth Web sites, and to develop and implement a customized protocol analysis that monitors and tabulates errors made by a person with cognitive impairment while accessing the Internet. Our long-term goal is to define the usability issues for individuals who are cognitively impaired and to develop specific Web usability guidelines. These recommendations for Web development will hopefully create a more universally accessible and usable environment that is more inclusive of people with cognitive disabilities.

\title{
References
}

[1] ODPHP, Healthy People 2010. At http://www.healthypeople.gov/. Accessed February 17th 2003.

[2] D. Krane, L. Light and J. Vine, 2000 N.O.D. / Harris Community Participation Study. New York: Harris Interactive; 2000. Study No. 12076.

[3] United States Census 2000. At http://www.census.gov/main/www/cen2000.html. Accessed February 17th 2003.

[4] W3C. The Web Accessibility Initiative (WAI). At http://www.w3c.org/WAI/. Accessed February 17th 2003.

[5] J. Nielsen, Designing Web Usability, (1st ed.), Indianapolis, IN: New Riders; 2000.

\section{[7]}

\section{Hygeia - An Innovative Information Technology-Based Program to Influence the Maternal and Child Health of Medically Underserved Families}

\author{
Michael R. Berman \\ Hygeia Foundation, Inc./Yale University School of Medicine, New Haven, USA
}

Hygeia(http://hygeia.org) improves the maternal and child health of the population it serves by reducing what are major disparities in health, particularly antenatal health care and thus reduce disparate rates of premature births, perinatal losses and infant deaths. Among these disparities is a lack of awareness of local healthcare facilities offering maternal and child health care services including prenatal care, a lack of understanding of the need for pre-natal care, albeit early care, the late diagnosis, recognition and acceptance of ones pregnancy, embarrassment and denial, and perhaps, I hypothesize, shame, diminished self worth and dignity and a feeling of isolation without a place or professional to turn to for immediate care, counseling and advocacy. These families must enter and be embraced by a healthcare system. Thus, by providing not only a significant awareness to the need for pre-natal care, but advocacy and personalized direction for this population at risk, such dignity and self worth can be realized, privacy 
and confidentiality assured, and through Hygeias Get Care program, personalized referrals are given to women to present at a healthcare facility so they will know there is an organization advocating for their health. Hygeia Foundation, Inc. now in its eighth year is an established and trusted Internetbased non-profit organization with a membership of more than 23,000 families, world-wide, currently providing online support, information and advocacy for families who have incurred the devastation of perinatal loss and infant death. The Hygeia Foundation, Inc., is unique in its ability to offer both a backbone of support and information for those families at risk for adverse pregnancy outcomes and now to work proactively to bring families into the health care system thus improving the individuals health and reducing morbidity and mortality. It has become a true system to provide an avenue for wide spread public access to the Hygeia Program through its portal, GetCare at Hygeia(http://getcare.info). This portal provides the user with a simple anonymous form which when filled out leads the user to a database of geographic-specific Federally Funded Clinics where Maternal and Child Health Services are available and Global Hospitals where prenatal and delivery care can be given. The user obtains a personalized, signed letter of referral from Hygeia, which introduces them to their health care facility as one who is a member of a respected and important global community and who needs help, much like a private patient would have a letter of referral to a doctor from another doctor. The user of this program has the advocacy of Hygeia to help and intervene so the user does get the care she needs. This is further supported by local and onsite, designated Hygeia Physician Advocates, selected from Ob-Gyn and Primary Care House Staff in participating Affinity Healthcare Institutions. The service is free of charge to those who wish to use it and can be easily linked to local and regional social services so the families can have their support as well. The primary entry point into the system is by a Hygeia Passbook which contains an autostart cd-rom business card which when placed in a computer converts the browser screen to a browser-less kiosk and goes directly to the Program. Likewise, this can be accomplished by going to the url: http://getcare.info. The CD/Hygeia Passbook is distributed at markets, fast food chains, pharmacies, laundromats, and other retail venues. The user goes to a public library, school, church, community center etc. (if no internet access is available at home) and presents the cd-rom card for assistance in accessing the GetCare program. A toll-free phone number is also offered. The program is offered in English and Spanish and will be expanded to other languages. The program serves a parallel purpose in narrowing the disparities of the digital divide and the disparities in access to healthcare.

[8]

\section{Availability on the Internet of Physician Consultations and Pharmaceuticals: A Reappraisal After Four Years}

Bernard S. Bloom ${ }^{\mathrm{a}}$, Miles J. Jones ${ }^{\mathrm{b}}$ and Ronald Iannacone ${ }^{\mathrm{c}}$

${ }^{a}$ University of Pennsylvania, Department of Medicine, Philadelphia, USA

${ }^{\mathrm{b}}$ USA Net Doctor International, USA

${ }^{\mathrm{c}} U S A$ SAP America Inc, USA

Introduction: Few systematic studies have been done of health care services available to consumers on the internet. In 1999, we quantified availability and cost of physician consultations and prescription medications through the internet. Our main conclusion was that easy access was balanced by higher costs of both. We found 46 websites worldwide; $80.4 \%$ required a prescription from a physician to obtain any medications. A limited number of prescription medications were available. We focused on sidenafil and finasteride, the only medications available on all study websites. We compared internet cost of physician visits to those for comparable visits to selected general internists, general practitioners and 
family practitioners in Philadelphia. Internet prescription medication costs were compared to those of local pharmacies in Philadelphia. Median cost of an internet physician visit in 1999 was $\$ 70$ compared to $\$ 60$ for a primary care visit in the Philadelphia region. Median per pill cost for sildenafil from internet sites was $\$ 5.49$ and among Philadelphia pharmacies it was $\$ 4.50$. For finasteride, median internet cost was $\$ 1.94$, while among local pharmacies it was $\$ 1.83$. Shipping and handling added $\$ 4.00$ to $\$ 25.00$ to internet service costs. The objective of this reappraisal was to determine changes in internet availability and costs of physician consultations and prescription medications in the intervening years.

Methods: We performed multiple searches to find websites offering physician consultations and/or prescription medications, 1 June 2003-31 August 2003. Data were collected from websites on geographic location and cost of physician visits and prescription medications.

Results: 194 sites provided physician services and/or prescription medications. Nearly all US sites, and one-third of non US sites, required or offered a physician visit. Mean payment for a physician office visit was $\$ 60$ in Philadelphia and $\$ 55$ for an internet consultation, $8.3 \%$ lower. Websites offered 160 individual prescription pharmaceuticals, but 204 medications when all doses were included. Mean cost per pill or per gram across all medications was $36.7 \%$ higher at internet sites than at Philadelphia pharmacies. Shipping and handling added a mean of $\$ 15$. We found 13 sites acting as pharmaceutical brokers to send customers to sites with the lowest prices. The charges were $\$ 10.50$ to $\$ 25$ for one month, or as low as $\$ 5.82$ per month if enrolled for more than one month.

Discussion: There has been a nearly 5-fold increase of internet sites offering physician visits and prescription medications, 10-fold increase of prescription medications available, and a doubling of medication classes available since 1999. Higher financial cost and risks accompany easy access to physician consultations and prescription medications. But higher costs may be acceptable tradeoffs for people living in rural or isolated areas, for those with limited mobility, or those who will pay for convenience. States are being asked to adjudicate interstate and international issues of physicians diagnosing and prescribing over the internet. Physicians have lost their license in individual states, and prosecutors have indicted and convicted physicians of practicing without a license. What remains unknown is real benefits, costs, savings and harm for patients through internet medical care, how much and by whom. Obviously, the value to patients and consumers is high, given expansion of the industry in just four years.

\section{[9]}

\section{ISABEL: The Use of a Web-Based clinical support system}

\section{J.S. Briggs and Tineke Fitch University of Portsmout, Portsmouth, UK}

Introduction: ISABEL [1] is a web-based paediatric clinical decision support system for use by healthcare professionals. The website is owned and has been developed by the ISABEL Medical Charity, a UK-registered charity. According to the website, the project started as a result of a little girl called Isabel falling dangerously ill with complications resulting from chicken pox. She had to spend 4 weeks in intensive care to save her life because her serious condition was not recognised in time. As a result of this bad experience, Isabel's parents led an effort to develop a resource that could be used by healthcare professionals to assist in the diagnosis of children.

Using proprietary pattern recognition software (Autonomy [2]) to search standard paediatric textbooks, a differential diagnostic tool produces a list of up to 15 diagnoses to consider for any given set of clinical features. Further decision support is provided by text, annotated images, and practice guidelines specific 
to each diagnosis. A section entitled "experience" attempts to capture and highlight common clinical lessons learnt at various steps within the guidelines and at relevant points in the diagnostic process [3].

The UK Department of Health $(\mathrm{DoH})$ is examining the potential effectiveness of the ISABEL website in the wider clinical context and exploring options for promoting its wider use in the UK National Health Service (NHS). This paper reports on work commissioned by the DoH to review the existing use of ISABEL.

Objectives and study design: The objectives of the study were to determine the extent of ISABEL's use, to find out user's attitudes to ISABEL and finally to identify impediments to its development. The study was done in two parts.

1. Web server log data was analysed to determine how many people actually use ISABEL and how often they do so. We examined two log files provided to us by the company that hosts the ISABEL website. The first was the conventional web server log. Analysis of this was performed using a number of web log analysis tools, including Sawmill [4]. The second was a user log recording validated logins to the site, plus new user registrations. This was analysed using custom analysis tools developed by the authors. Both covered the period from when ISABEL was first set up (18th July 2001) to when our survey ended (19th December 2002).

2. A 24-item questionnaire was sent by email to 4436 of the 7179 registered users of the system. This was designed to determine user attitudes to the system. Since the review concerned ISABEL's use with respect to its impact in the UK, the questionnaire was sent only to those whose registration details showed them to be based in the UK. A letter accompanied it from the ISABEL team encouraging recipients to complete the form. Returns could be sent by email, post or fax.

Results of the web survey: The web survey showed that of the total number of users (7179), 3330 (46\%) had only used ISABEL once, 5186 (72\%) used it no more than twice, and 6493 (90\%) used it no more than 5 times. However there was a core of approximately 50 users who had used the system more than 25 times, with an average frequency of one week between visits.

Usage was highest during the weekday working day, with reduced usage out of normal hours and at weekends.

Most usage (where identifiable) was from the UK, with at least a quarter of accesses coming from somewhere within the NHS, but there was also a significant amount of access via Internet service providers. A detailed analysis of precisely where in the NHS users were located proved to be impossible because of anonymisation implemented by NHS firewalls.

Results of the user questionnaire: 523 responses were received (11.8\%) of which 518 (11.7\%) were completed sufficiently for analysis. Of particular interest to the study for the purposes of analysis was the distinction between responses from those who were paediatric specialists (58\% of respondents) and those who were not $(42 \%)$.

As expected, the paediatric specialists used ISABEL slightly more frequently than non-specialists. Three-quarters say they would use it more but for time constraints and lack of access to information technology. For those who would not use ISABEL more often, the main reason is that they use ISABEL as much as they require. However, one-third of paediatric consultants and one-fifth of General Practitioners say ISABEL is unsatisfactory in its present form and improvements are needed.

The aspects of ISABEL that are particularly liked are the differential diagnostic tool and the guidelines, and approximately two-thirds are of the opinion that ISABEL assists in clinical management.

Discussion: Findings suggest that there is a small and very supportive community of devotees, including those who are involved in the development of the site, but that the website is either not 
sufficiently useful or not sufficiently convenient for the vast majority of users who have tried it out. The reasons for the latter are largely systemic to the NHS and the way it delivers its services, and would require considerable resources to make more Internet-connected computers available, and to give staff more time and opportunity to use them.

The website itself exhibits some good properties: there is little evidence of broken links or changing structure. Most output is dynamically generated using Java Server Pages but one criticism of the site is its use of frames, which are deprecated by most web page experts. Their adoption on this site has made some analysis of ISABEL's usage (such as how users proceed through the site) impossible.

\title{
References
}

[1] ISABEL, website available at http://www.isabel.org.uk (Accessed July 2003).

[2] Autonomy, website available at http://autonomy.com (Accessed July 2003).

[3] P. Ramnarayan and J. Britto, Paediatric clinical decision support systems, Archives of Disease in Childhood (November 2002).

[4] Sawmill website available at http://www.flowerfire.com/sawmill/ (Accessed July 2003).

[10]

\section{Consumer Informatics: The Elderly and the Internet}

\author{
Robert James Campbell \\ Duquesne University, Pittsburgh, USA
}

The Internet is one important way for health consumers to participate actively in their health care. This new found ability has lead more and more consumers to question, not only the type of care they receive, but also the quality of that care. However, as Internet usage continues to grow, differences in how demographic groups use this resource to locate health related information are becoming more pronounced. Review of Literature A recent survey by the University of Pittsburgh (2000), shows that $62 \%$ of the residents of Pittsburgh and surrounding Allegheny County have access to the Internet. However, the average older adult in Allegheny County, the second largest population of older adults in the nation, has the lowest levels of computer ownership and more limited access to the Internet than other county residents.

Furthermore, these elderly adults, who make up $17.8 \%(228,416)$ of the countys $1,281,666$ residents lack the essential knowledge of how to use the Internet to locate health information. Nationally, research shows that older Americans are in danger of being cut off from one of the most provocative communication mediums of the 21 st century. In the United States, elderly adults make up $13 \%$ of the population with only $4 \%$ using the Internet. Overall $56 \%$ of America is online and out of that percentage, only $15 \%$ age 65 and over have direct access to the Internet (Fox and Rainie, 2000; Fox, 2001; Fox and Rainie, 2002). 93 million American adults use the Internet to locate health related information, with only 4 million aged 65 and older (Fox and Fallows, 2003). Because older adults are more likely to suffer health problems and make frequent use of the health care system (AOA, 2001), knowledge of how the Internet can be used to locate health information to manage ones care would benefit senior citizens. By manage, it is assumed that individuals will use the Internet to investigate illness states, treatment regimens, the background and training of their physicians, and the efficacy of taking specific medications.

Personal Health Care: Why Get Involved? The literature shows that the majority of patients prefer to leave their medical decisions to their physicians (Kaplan et al., 1996; Arora and McHorney, 2000). 
Not only is this the case for older and less educated patients (Ende et al., 1989; Frosh and Kaplan, 1999; Benbassat, Pilpel and Tidhar, 1998; Bilodeau and Degner, 1996; Kaplan et al., 1995; Beisecker, 1988), but also physicians perpetuate this trend as their experience and education increases (Beisecker et al., 1996; Paterson, 2001). Over the years, studies have shown that patients over 60 years of age have a lower desire for control over their health care when compared to younger adults (Stiggelbout and Kiebert, 1997; Smith et al., 1988; Woodward and Wallston, 1987; Cassileth et al.,1980; Haug, 1979). Moreover, older patients and men are more likely to let the physician make decisions regarding their treatment (Breemhaar, Visser and Kleunen, 1990). This suggests that the elderly tend to have an external locus of control when it comes to health beliefs (Caress, 1997), as opposed to an internal locus of control. Although the majority of patients prefer to let their physician make the decisions, the more a patient learns about her illness, the more likely she is to ask questions of her physician (Frederikson and Bull, 1995; Mullen, Main and Velez, 1992; Kaplan, Greenfield, Ware, Jr., 1989; Sharf, 1988; Mullen, Green Persinger, 1985). Studies have shown that patients who ask questions, elicit treatment options, express opinions, and state their preferences regarding treatment during office visits with their physicians have measurably better health outcomes than those who do not communicate (Kohn Corrigan and Donaldson, 2000; Rost et al., 1991; Mahler and Kulik, 1990; Barry et al., 1988; Greenfield et al., 1988; Greenfield, Kaplan and Ware, Jr., 1985).

Reaching Older Adults: Beginning in 2001, a program was developed at Duquesne University to train older adults how to use the Internet to manage their health care. The program consisted of five one hour and half sessions presented over a five week period. The sessions were held in local public libraries and senior community centers in Pittsburgh and surrounding communities. The sessions included basic instruction on how to use a computer and web browser, search engines, evaluating web sites, and learning how to use specific medical web sites to answer health care concerns. Along with the instructional component, the program measured participants health locus of control, levels of self efficacy, opinions on health care, and their levels of participation in their health, both before and after taking part in the program. Thus far the program has trained close to 200 older adults.

The program has now partnered with the Carnegie Library of Pittsburgh, and has received a grant from the National Library of Medicine to continue to training older adults in the Greater Pittsburgh area how to use the Internet to manage their health care. This presentation will explore the relationship that exists between the Internet to the elderly and the factors that may influence their use of the Internet as a resource for locating health information. Moreover, the presentation will explore the impact that Internet training has had on the elderly with regard to their participation in their own health care. Did they view the Internet as a novelty, something to fear, or did they express a desire to use the Internet to become more informed health consumers, and as a more informed consumer, did they desire a more participatory role in their own health care, or did they remain passive?

[11]

\title{
COCOON: How to reduce risk in medicine
}

\author{
Vincenzo Costigliola \\ European Medical Association, Bruxelles, Belgium
}

COCOON is an integrated project aimed at supporting health care professionals in improving risk management in their daily practices by building knowledge driven dynamically adaptive networked communities within European healthcare systems. Risk management for a health care professional is governed by the responsibilities assumed in making patient diagnoses and ordering treatments. The 
growth of individual patients' capability of judgment and access to the necessary information together with a prior cost/benefit analysis in order to decide on possible medical interventions are three important factors that force the health care professional to pay much more care in providing the correct answer to the patients' problems. In the last 5 years the tenfold increase in legal cases and the fivefold increase in health care insurance premium costs for the health care professional for the cover of medical errors, are two important indicators of the relevance of the problem at the European level.

The technical objective is to develop a Toolset of interoperable, scalable and reusable Web Services supporting Community and Knowledge Management practices in a wide range of networks of healthcare professionals.

\section{[12]}

\section{Reuse and adaptation of an open source digital campus for general practice e-formation}

Marc Cuggia, Charles Honnorat, Sahar Bayat and Pierre Le Beux

Laboratoire d'Informatique Medicale, Faculte de Medecine de Rennes, France

Introduction: E-learning is defined as the use of network technologies to create, foster, deliver, and facilitate learning, anytime and anywhere. In France, the training of general practitioners presents several characteristics which justify e-learning: Geographical dispersion of teachers and especially students (who have to carry out six clinical training courses in several hospitals and at the general practitioners' offices); Diversity and multiplicity of optional courses; Necessity of training the future practitioners to use information and communication technologies. In this context, the Medical Informatic Laboratory (MIL) in collaboration with the Department of General Practice (DGP) of Rennes University (France) set up an e-learning platform for the training of medical students.

Materials and methods: LIM decided to use an Open Source campus for several reasons: low cost, accessibility of the source code allowing an adaptation to the local needs and software continuity. We choosed Claroline campus (developed by UCL Belgium). Development of this tool is focused on Nielsen principles of Web Usability which means that the tool is very easy to use. Furthermore, its not based on proprietary technologies. This fact guaranties its technological independence. Claroline is used by numerous universities. The Open Source community which contributes to its development is particularly active.

Results: The general practice campus has been validated by professors and students during last year. Its now accessible by this URL: http://www.mgcampus.com. This platform allows pedagogical resources and multimedia documents to be put on-line. Students can consult their training program on the Web. Professors can organise e-learning teaching either for all of the students or for small groups. Feedback between students and teachers can be carried out by forum, email and chat. Finally, the platform offers several tools for students' evaluation.

Conclusion: We reused the Claroline platform and adapted it to the specific needs of medical teachers and the students. Specific developments have been facilitated by exchanges with the other members of the Open Source community. We achieved an important objective of this project which was ensuring the autonomous management of the platform by the DGP. Acceptability and utilisation rate of this campus will be evaluated during the next year. Whatever the technological tool's performance may be, success of an e-learning project is based mainly on pedagogical methods and content used for training.

\section{[13]}

The Personal Health Record as part of a Personal Health Information Infrastructure and Its Relationship to a Comprehensive Health Information Infrastructure 
Mary Jo Deering, Ph.D.

Deputy Director for eHealth and Management, Washington, USA

Office of Disease Prevention and Health Promotion

US Department of Health and Human Services ${ }^{1}$

Within the United States and some other countries, there is increasing interest in "personal health records" (PHRs), which are broadly defined as containing the personal health information of a given individual across plans, providers, and, potentially, a lifetime. Draft functional standards for a personal health record, including its relationship to medical records in specific care settings, are being developed by HL7, a major international health standards-setting body, in the Fall and Winter of 2003. At the same time, the US and other countries are pushing strongly to develop national health information infrastructures (NHIIs) to enable the sharing of clinical information to improve healthcare quality and outcomes. While most NHII-related efforts recognize the role of the consumer, patient, or caregiver, most of the activity and funding is directed to providers and institutions. This presentation will describe the purposes, functionalities, and current uses of PHRs, and the barriers to their diffusion. It will describe the nature and functions of a personal health information infrastructure that includes a PHR but also customized access to providers, health information, care plans, and personal support; and which also enables linkages to provider-based systems. It will describe the critical role such an infrastructure can play in: improving patient safety and health outcomes; strengthening continuity of care; increasing the ability of consumers and patients to understand and manage their own health and to understand, affect, and participate in the care they receive from professionals; and increasing the efficiency and effectiveness of healthcare systems. It will describe the barriers to the development of such personal health information infrastructures and steps (within health policy and practice) for overcoming these barriers.

\section{[14]}

\section{Patients use of health information on the Internet: The impact on primary care consultations}

Gina Catherine Dolan, Rachel Iredale, Robert Williams and Jamal R. M Ameen University of Glamorgan, Pontypridd, UK

Introduction: Patients use of health-related information from the Internet is increasing at an exponential rate. Although some information is available about how health professionals use the Internet (Roscoe, 1998, Wilson, 1999) little is known about how patients utilise this information. Some patients may actively seek information to assume more responsibility for their health. However, others may feel obliged to do so because of failing confidence in health care provision. An increasing number of patients bring in information which they have accessed from the Internet for discussion in primary health care consultations. Such assertions are primarily reported from GPs experiences in consultations rather then directly from patients.

This clearly supports the move towards patients taking on a more active role in consultations. Health professionals have the potential to assist patients to make sense of health information from the Internet; however they may not necessarily welcome this role (Wilson 1999, Murray et al. 2003). Aim This study aims to evaluate patient use health information from the Internet in primary care and consider the

\footnotetext{
${ }^{1}$ The views expressed in this abstract or presented in the final presentation do not necessarily reflect the views of the Office of Disease Prevention and Health Promotion or the US Department of Health and Human Services.
} 
impact of such use on the doctor-patient relationship. Study design Adult patients $(n=50)$ from general practices in South Wales (UK) were interviewed about the health information they use, including the Internet. Healthcare professionals $(n=12)$ were also interviewed about how patients use the Internet and the impact this has on consultations. To address a potential digital divide the practices were selected from areas with differing levels of deprivation.

Results: Patients were positive about using the Internet as a source of information. Use of the Internet was mainly triggered by an illness or condition, and in some cases patients turned to the Internet for information about an unresolved health problem. Patients often sought confirmation of Internet information by discussing it with their health professional at a subsequent consultation. Discussions of this kind were highly valued by patients who often felt more in control and satisfied with their consultation. However, there was a general concern by patients that health professionals would feel challenged by the introduction of such information, as they would feel that their role as a professional was being questioned. In some cases this discouraged patients from referring to Internet information in consultations.

Health professionals were generally supportive of informed patients but a major concern was the time required to discuss information brought in by the patient. Conclusions Many patients actively use the Internet as an information source. The use of Internet information during consultations is on the increase, and although patients are positive about such use, doctors do not necessarily encourage Internet informed patients. It is now important to consider perhaps the changing role of health professionals how patients can be best guided in their use of health information in the future.

\section{[15]}

\section{An interoperable, graphical environment for the capturing of medical information}

Fefie Dotsika ${ }^{\mathrm{a}}$ and Andrew Watkins ${ }^{\mathrm{b}}$

${ }^{\mathrm{a}}$ University of Westminster, United Kingdom

${ }^{\mathrm{b}}$ University of London, United Kingdom

From pathology, diagnostics and treatment, to patient history and lifestyle, medicine is a true science of information. As medical information is growing, its management and utilisation becomes more challenging. While the current generation of electronic healthcare applications keeps on multiplying, doctors, patients and medical administrators are faced with the task of choosing the right application that will enable them to find and use the relevant information at the right time.

Resulting from the recent experimental deployment of functional database management systems for the storage, manipulation and retrieval of medical information [1,2], MedISD (Medical Information System Design) has been developed, a web-based, graphical, information modelling environment, which enables practitioners to model their own custom-made healthcare information systems. The development of MedISD was deemed necessary following the agreement for the trial use of the system with NHS primary healthcare data.

MedISD focuses on improving healthcare practice by enabling custom schema modelling, direct representation and flexible use of medical knowledge, and support of metadata and multimedia content.

The aim of the system is thus to significantly reduce the complexity of developing medical information systems, from primary healthcare data pools to distributed e-health applications. No technical knowledge or database expertise is required apart from basic desktop environment skills. The tool captures information in the form of directed graphs and automatically generates tailor-made medical database schemas based on the functional data model. The system supports complex objects, user views and it is further integrated by providing an XML interface that allows for interoperability with other databases and medical knowledge repositories in general. 


\section{References}

[1] F. Dotsika, Modelling medical operational knowledge for e-health applications, International Journal of Health Care Engineering, Technology and Health Care 10(6) (2002), IOS Press, Amsterdam.

[2] F. Dotsika, From data to knowledge in e-health applications: an integrated system for medical information modelling and retrieval to appear in the International Journal of Medical Informatics and the Internet in Medicine.

[3] F. Dotsika and A. Watkins, Integrating Web-Based Information Systems: WWW and the Functional Model, Proceedings of the IASTED Conference on Internet and Multimedia Systems and Applications IMSA2002.

[16]

Internet and Patient Education: The current evidence for chronic disease management

Persephone Doupi and Pekka Ruotsalainen

Centre of Excellence for Information and Communication Technologies, STAKES, Finland

Objective: The Internet has been acknowledged and established as a powerful channel for the distribution and communication of health-related information, therefore raising the interest for its adoption in patient education strategies. We undertook a systematic review of the literature in order to identify evidence of the effectiveness of Internet use in patient education programs, targeting specifically clinical domains with a chronic course (such as diabetes, asthma, cardiovascular diseases, dermatological conditions, etc.). Particular emphasis was placed on the technological features of the applications involved and on the considerations undertaken in the process of their design and implementation, as critical success factors for these interventions.

Methods: Data sources: We devised a search strategy on the basis of MeSH subject headings, to broadly cover the areas of patient/health education, patient and consumer participation, disease management and Internet applications. We initially used this strategy to search MEDLINE, as a primary health literature resource.

Study Selection Criteria: In order to be considered for review, identified publications should: address patient education interventions aimed at a chronic disease or condition; target any age group of patients and/or their carers or family members; involve the use of Internet-enabled applications ; report on a randomized controlled trial, controlled clinical trial or clinical trial or be validation or evaluation studies or meta-analysis. Publications addressing acute or temporary medical conditions, dental care, general health promotion or preventive interventions targeted at healthy individuals were excluded. Additionally, we excluded interventions targeted exclusively at health care professionals, as well as opinion or viewpoint papers, commentaries and publications discussing methodological issues of patient education.

Results: Our search of MEDLINE (1966-2003) retrieved an initial set of 3443 items in total. Limiting the results only to items with an abstract and to publications concerning human subjects reduced the set to 2408 items. Selecting only items with the publication types listed in the inclusion criteria resulted in a set of 264 articles. We are currently processing this subset, extracting information on the following areas: a) general information (year of publication, country, type of study, clinical application domain); b)information regarding the patient education intervention (based on a previously proposed taxonomy for patient education research reporting and on research in the field of chronic conditions management); c) information regarding the IT tools and infrastructure used in the intervention. Further study selection is in progress, through adaptation and use of the search strategy across additional relevant resources, such as CINAHL, INAHTA-HTA, the Cochrane Collaboration database etc. 
Conclusion: The application of the Internet in patient education is still a largely unexplored research area. The systematic literature review we have undertaken aims at providing an overview of the currently available evidence regarding the effectiveness of Internet-enabled patient education strategies, particularly in the context of chronic disease management. An analysis of the findings that correlates effectiveness with specific system characteristics, as well as with the features of the clinical application domain can provide valuable input for the design and development of patient education systems, as well as for the successful incorporation of the Internet in patient education programs.

\section{[17]}

\section{Lose fat through the internet? The quality of weight loss related websites}

Julia Eckl-Dorna and Ernest Groman

Institute for Social Medicine, Vienna, Austria

The internet and the problem of obesity have one thing in common: They both showed an enormous growth within the last decade. Overweight people, who desperately try to lose weight, may often use the anonymity of the internet to find help. This study evaluates the websites on weight loss that were found in the ten most used search engines by entering the search strings weight loss and diet.

The first three pages of results ( 10 results per page) were included into the study. The websites identified were subjected to a quantitative(categories) and qualitative analysis (IQ-Tool, Voluntary guidelines for providers of weight loss products or services). Out of the 600 search results revised, 258 different webpages were identified.

The author was identified in $23.1 \%(n=46)$. In $14.6 \%(n=29)$ of the websites we could determine the sponsor, in $0.5 \%(n=1)$ financial conflicts were explained and $2 \%(n=4)$ stated, that sponsors have no control over the content. 28.6\% $(n=57)$ contained a date of last update and 52.6\% $(n=30)$ of those were current (defined as not older than 6 months). $70.4 \%(n=140)$ followed less than $1 / 3$ of the guidelines, $27.6 \%(n=55)$ integrated $1 / 3$ to $2 / 3$ in their websites and $2 \%(n=4)$ followed more than $2 / 3$ of the guidelines. The quality of the websites is very poor and voluntary guidelines are seldom applied by the producers of webpages. Tools for evaluating websites like the IQ-Tool are not well known by consumers and it takes time to check every site you are interested in. Therefore other ways of controlling quality in the internet like creating labels or using portals should be considered and advertised.

[18]

Use of and Trust in Health Information on the Internet among the Finnish Population

Stefan Ek

Abo Akademi University, Abo, Finland

The present day society has a potential and pronounced strategies for a development where global information networks and interactive media constitute positive tools in view of a healthier and more active life for every citizen. The optimists in late modern society claim that the gap between the information rich and the information poor will diminish as the WWW-culture keeps spreading. Optimistic decisionmakers consider this as a new opportunity to create comprehensibility, manageability, meaningfulness and healthier life styles across all social classes. The Internet has a big potential as an empowering tool and a great equaliser, putting the socially alienated groups of citizens on an equal footing with other people. However, it is also predicted that the Internet might broaden the social inequality and widen 
social gaps. The digital divide might cause the disadvantaged groups to become increasingly left behind. It is to be feared that the population groups who are unable or unwilling to use modern information technology will be isolated from the increasingly predominant way of communicating, working and living in the society.

Elderly and persons belonging to the lower social groups use the Internet less than younger people and socially more established groups, but the reason has been found to be mainly access difficulties. The problem is expected to be attenuated as the use of the web stabilises. As more people will be connected, many of the health-promoting interactions with the citizens will take place through electronic devices rather than through personal contacts and the Internet has, indeed, quickly become a communication technology used by an estimated 408 million people worldwide. Electronic technology affects personal information infrastructures at all levels: physically (different motor movements), cognitively (complexity and speed), and emotionally (stress and intellectual productivity). It offers a vast amount of information regarding health and medical issues, with web sources representing the whole spectrum from reputable and controlled medical information - offered through governmental or educational gateways - to uncontrolled and uncontrollable web sites of a personal, commercial or ideological nature. Additionally, professional medical literature is available free in various full-text electronic journals, as well as on the Medline database, which contains abstracts from over 4000 peer-reviewed journals. The primary purpose of most health sites on the web is consumer education and marketing. In August 2000 an estimated 40.9 million people sought health information on the Internet and in 2005 their number is predicted to reach 88.5 million.

Particularly the use of the Internet in the health education of the population has been widely acknowledged. When the capacity of the web will further increase, the Internet is predicted to become the main link within professional medical groups and between them and the citizens. According to predictions medical problems will often be dealt with at home and the treatments will become preventive rather than crisis solving.

Within the field of public health information, mass media channels have proven capable of reaching and informing large audiences, but when it comes to changing attitudes and modifying health practices, interpersonal channels have been more successful, as they provide immediate feedback and social support. The Internet can, in fact, be seen as a hybrid channel, combining the positive attributes of both interpersonal and mass communication. It thus becomes more than merely a new means of disseminating medical news; it is also an information environment where the traditional roles and interrelationships of medical professionals, journalists, corporations and ordinary citizens are changing; and where the health information consumers enjoy increasing control over the selection, dissemination, and even the interpretation of health news.

\title{
[19]
}

\section{Swiss Orthopaedic Register "Pilot" - Technology \& Methodology}

\author{
Amer Ibrahim EL-Kerdi, Christoph Roder and Urs Muller \\ University of Bern, Switzerland
}

Documentation is at the core of quality assessment. To deliver an objective as well as scientifically valid judgment of implant and treatment performances, all relevant data must be centrally captured where and when it is generated by the concerned individual(s) in the clinical pathway. The Swiss Orthopaedic Register (SOR) pilot is a project of the Swiss orthopaedic community to assess the establishment of a nationwide centralized data collection, archiving, and distribution system in potentially 190 Swiss 
orthopaedic clinics. The SOR technology provides as much flexibility and modularity as possible to users and participating clinics to ensure acceptance. The solution integrates a full documentation system in a centralized setup with clinical, surgical, implant, radiological, as well as patient datasets. This modular technology facilitates the generic integration of three distinct yet complimentary solutions: an internet application based on the most advanced web technologies, a networked remote paper solution using Optical Mark Recognition (OMR) scanners, as well as a mobile data collection interface using barcode scanners. The SOR is also empowered with several propriety external interfaces that integrate authoritative third-party systems, such as a web content management system, a standardized faulttolerant Digital Imaging and Communications in Medicine (DICOM) compliant Picture Archiving \& Communications System (PACS), a barcode implant data capture solution for medical device tracking directly from hospital operating rooms, as well as a statistical engine powered by SAS. All external systems are integrally combined with all online \& offline data collection, archiving, and distribution mediums. Direct rewards associated to this e-healthcare solution relate to the long-term electronic monitoring and evaluation of the various implants and procedures in the Swiss orthopaedic community. The centralized data archived in the electronic documentation system will form the basis for improving the efficiency and outcome of orthopaedic surgery, rendering feasible a centralized data management e-healthcare system, and enabling a cost-risk in addition to cost-efficiency analysis of new medical devices and treatments. In essence, the implemented e-healthcare solution is an essential tool for the maintenance and improvement of orthopaedic patient care. The SOR will also constitute a crucial tool to help regulate the inflating costs of the Swiss healthcare systems by scientifically demonstrating the benefits and adverse effects of currently used as well as newly introduced medical technologies and treatments. These have a direct influence on the economical viability of orthopaedic surgery today and in the future.

\title{
[20]
}

\section{A Framework for Medical Costanalysis in XML and Cocoon}

\author{
Christian Elsner ${ }^{\mathrm{a}}$, Christian Mazzi ${ }^{\mathrm{b}}$, Gerhard Hindricks ${ }^{\mathrm{a}}$ \\ ${ }^{\mathrm{a}}$ Heart Center Leipzig, Germany \\ ${ }^{\mathrm{b}}$ US Click4Care, 1, Germany
}

Cost-Effectiveness Analysis becomes an important tool for Providers and Payers in establishing new therapeutic options for patients. Despite a huge amount of published Information on Evidence based Data on Cost-Effectiveness is available over Medical Databases (e.g. MedLine) there is a huge time gap in "time to provider" and "time to payer" of this information. Objective of our work was to design a concept and data structure for easy and semi-automated set up of Cost-Effectiveness Markovmodels for rhythmologic topics and evaluate those with a portion of available literature. The Tools for Concept and Data structure were built using a XML-Designtool from Altova Inc. and a Visual Basic Program. A first alpha-prototype of a markov node representation layer webfrontend with condensed reference information was built with a Tomcat / Cocoon 2.0 Framework. For the Evaluation of the Model we used Review / Original Literature from Carol et al. on Cost-Effectiveness of Ablation for Supraventricular Tachykardia and Literature from Eckman et al. and Weerasooriya et al. on Cost-Effectiveness of PAF Treatments. As a result of our Work we developed two XML-Schemes, one to represent EBM / Cost-Effectiveness relevant information (Demographic, Clinical, Costs, Utilities and Disutilities) from Publications (= MedEc-XML-REF) and a second to map and weight this information to defined markov nodes (MedEc-XML-MAP). With the System 60 References could be mapped into the new Data structure. 
With the prototype in Tomcat / Cocoon 2.0 we could rebuild the models from the literature graphically. The System could then give a web-enabled fast overview of the underlying referenced information and Clinical Evidence of the Data per node (for the Evidence Rating we used a modified model from US Preventive Services Task Force). At least new data could be added fast through the integration of XML-structured data from a number of (fictive) new publications for evaluation purposes and validation of the system. The developed data scheme seems to be feasible to extract relevant information from the publications. Mapping the Information from the references still needs sort of interpretation which needs to be commented in both the schemes. A further granulation of the XML schemes may therefore be necessary. The process of mapping reference information to the markov nodes is highly enhanced due to semi-automation by the use of common classification codes for procedures and outcomes. The principle of the graphical representation may be useful for consecutive literature search / investigations being performed for further evidence of Cost-Effectiveness at certain points.

[21]

\section{Patient perceptions about communicating with a nurse through webchat}

Nina Eminovic and Jeremy C. Wyatt

The Netherlands Academic Medical Centre, Amsterdam, The Netherlands

Objectives: NHS Direct is a British telephone triage service which the public uses to contact a nurse for any kind of health problem. This service, funded by National Health Service (NHS) receives 7.5 million calls a year. NHS Direct Online2 (NHSDO; www.nhsdirect.nhs.uk) is an extension of NHS Direct where the telephone is replaced by the Internet, introducing new possibilities in informing the patients about health. One of the NHSDO services in development is Clinical Enquiry Service (CES) which uses webchat as the communication medium. We carried out the first evaluation of this nurse-led webchat triage service, in the form of a pilot study to explore the feasibility and safety of and patient perceptions about using webchat to contact a nurse. In this abstract we discuss changes in patient perceptions.

Study Design and Methods: The 6-day pilot was performed in an inner-city general practice in Coventry. Non-urgent attending to see their GP patients were asked to participate and sign an informed consent. As soon as a patient agreed to participate, they filled in the first TMPQ (Telemedicine Perception Questionnaire) form 3 to capture their impressions about CES before using it. After this, they started the webchat by answering questions about their general health. The webchat with a trained NHS Direct nurse based in Southampton ended with advice about whether the patient should visit a health professional and how soon. Soon after, the patient filled in a second TMPQ form and an exit survey. All patients saw their GP after using CES for a consultation and treatment.

A literature search for a validated instrument to measure patient attitudes toward telemedicine identified the Telemedicine Perception Questionnaire (TMPQ), from the University of Minnesota, which has a maximum score of 60 points. With permission from the copyright holder, we adapted the questionnaire for our study, removing financial questions and changing "telehome care" into "webchat". Our questionnaire consisted of 12 items to which a patients could respond with their level of agreement (1: strongly disagree, 5: strongly agree). Data analysis was performed using SPSS 10.1.

Results: 25 patients from a wide range of ages (range 19-81, mean 48) were included in the study. Both pre and post TMPQ forms were filled in by 21 patients. The mean total pre use TMPQ score was $44 / 60$, increasing significantly after CES to $49 / 60(\mathrm{p} 2$-tailed $=0.008)$. Encouragingly, there was no correlation in either TMPQ score with the age of the participant. After using webchat, patients seemed less concerned about privacy and lack of face-to-face contact. There were statistically significant changes 
in patient perceptions about CES becoming a standard way of health assessment in the future (p2-tailed $=0.009)$ and CES making it easier to contact NHS Direct (p2-tailed $=0.002$ ).

Conclusions: Although these positive TMPQ results encouraged the NHS developers to continue with their work on CES, other aspects such as safety and cost need to be explored further before continuing the development process.

\section{[22]}

\section{Ethical and Legal Aspects of Using Digital Images of Patients for Learning and Teaching}

Jill Evans and John Mahoney

University of Bristol, Bristol, UK

Introduction: The BioMed Image Archive project (http://www.brisbio.ac.uk/) is leading an investigation of current practices in the biomedical communities regarding the acquisition, treatment, publication and use of digital images of patients for learning, teaching and research. The project is particularly focusing on the ethical and legal issues involved. Currently, the Archive contains approximately 8500 images that are free for use in non-profit educational contexts. Images have been donated by medical, dental and veterinary science academics worldwide.

At the time that these images were collected (mid-1990s), it was not always practice to obtain the permission of the patients involved. This was (and in some cases still is) particularly the case where images were likely to be completely unrecognisable, for example, a tissue sample or an x-ray. However, in the last few years, following a number of high-profile incidents involving the abuse of patients' rights and the rights of relatives of patients, the medical profession has become far more aware of and sensitive to the human and moral rights of all concerned in the recording of images for educational purposes. It is now increasingly common practice to obtain the informed consent of a patient before taking any kind of image that is intended for educational use.

Additionally, recent changes in UK human rights legislation, coupled with several notorious examples of the abuse of individuals' moral rights in various arenas, has brought the issue of the publication of images of people on the Web to the forefront of educational debate, and in some cases has created a climate of fear and bewilderment. There is a danger this uncertainty and apprehension may lead to the removal and destruction of visual resources that are (by their very nature) sensitive. In this worst case scenario, medical education could restricted and diminished.

Education has traditionally been seen as somewhat exempt from the concerns of the commercial and industrial worlds because of the widely-perceived altruism and benevolence of its pursuits but these assumptions can no longer be taken for granted.

\section{[23]}

\section{The Internet: Issues for community care}

\section{C.J. Fitch, C. Adams and J.S. Briggs \\ University of Portsmouth, Portsmouth, UK}

The theme of this 8th MedNet conference is Internet in Health for All and one area that exemplifies health for all is Community Healthcare (CHC). This paper examines CHC in the UK, which covers health and social care systems. Social care in the UK is "usually defined as long-term domiciliary care for elderly people, people with mental illness, and people with learning difficulties" (EOHCS 2002). 
The "community" aspect of CHC means that it could benefit from the many opportunities offered by the Internet for service delivery and organization in health and social care. It can be used to assist the distribution of medical information to parties concerned, such as electronic patient records and electronic mail and provide information for medical education and patient information.

The Internet could also support mobile applications in CHC and its healthcare professionals. However, there are many challenges that need to be addressed to ensure such applications and services are achievable, usable and useful. This paper examines the scope and potential for the use of the Internet to assist $\mathrm{CHC}$ professionals when using mobile devices and discusses the issues and challenges facing Internet-based mobile service provision, its management and practical issues. These challenges are not solely technical but also relate to organizational and social issues and several are focused more on reengineering the supporting processes and work protocols than on particular technologies.

This paper has been developed from an investigation (still ongoing) of mobile technology use in community care in a region in the South of England which has shown that CHC is a complex heterogeneous environment, often involving patient/client based ad hoc activity drawing upon different services within healthcare as well as groups outside such as housing and social services. There are issues concerning support structures, service management and organization and additional challenges are caused by the fact that CHC typically has to operate within political frameworks at national and service levels.

The paper lists a number of issues that need consideration if the use of Internet-enabled mobile devices is to become a viable tool for the CHC professionals; the main issue highlighted in this study is the tension between what the professionals "on the ground" require and what the Government perceives is required.

\section{[24]}

\section{Evolution of Medical Informatics teaching in a medical undergraduate course}

Alberto Freitas, Ricardo Correia, Filipa Almeida and Altamiro Costa-Pereira

Department of Biostatistics and Medical Informatics, Faculty of Medicine, University of Porto, Portugal

Introduction: The Department of Biostatistics and Medical Informatics (SBIM-http://sbim.med.up.pt) is a department of Faculty of Medicine, University of Porto, Portugal. SBIM, created in 1996, conducts teaching and research, and provides services in the fields of biostatistics and medical informatics. SBIM is responsible for the teaching of a first year discipline "Introdução à Medicina" (Introduction to Medicine) of the courses of Medicine (Faculty of Medicine) and Dental Medicine (Faculty of Dental Medicine). This discipline covers the areas of biostatistics, research methodology, medical informatics, bioethics and history of medicine.

The field of health and medical informatics education is of particular importance at the beginning of the 21st century: training and education in medical informatics is required in order to provide good quality health care [1].

In the medical informatics programme of our discipline, we try not only to track the evolution of technology but also to follow several other suggestions such as van Bemmel's [2] and IMIA [1] recommendations.

With the creation of groups for the study of specific subjects in medical informatics we intend that our students meet the potentialities of medical informatics and also that they perceive the state of medical informatics in Portugal.

Initially, teachers present possible medical informatics topics, but students can suggest other subjects of their interest. One teacher supervises each group with approximately 6 elements.

The work is annual and has the following sequential operational phases: 
1) Definition of the question: what do we intend to study?

2) Bibliographical search on the subject

3) Definition of the type of study

4) Definition of the target population and sampling method

5) Definition of the questionnaire to be used

First presentation of the work (4th month)

6) Definition of data collection methods

7) Creation of the SPSS database

8) Data collection

9) Data introduction in the database

10) Data analysis

11) Second presentation of the work (7th month)

12) Writing of the scientific article

13) Construction of a web site

14)

Objectives: With this work, we intend to show the improvements in our medical informatics education and teaching.

Methods: Regarding the theoretical component, we studied the evolution of the medical informatics topics covered in our discipline for the last four years. They were grouped into generic themes covering the areas of informatics and medical informatics. Each theme represents at least one-hour lesson.

Concerning the practical component, we analysed the number of students and teachers involved in works related to medical informatics from the beginning of such works, two years ago. Like the topics covered in our discipline, the works were also grouped on the same themes.

Results: In 1999, the discipline programme covered 3 topics of Medical Informatics ("Medical bibliographic databases", "Standards, coding and classification", "Telemedicine") and 4 topics of General Informatics. This equilibrium between both major areas translates the concern of the lack of computer skills by first year students.

In 2000, only 3 topics of General Informatics were covered (mainly basic Internet skills), and the Medical Informatics programme was expanded to include "Medical Imaging", "Security, ethics and legislation" and "Clinical Decision Support Systems".

In 2001, the General Informatics programme was reduced to one theme, while the Medical Informatics grew to cover 2 more themes ("Electronic Patient Records" and "Future of Medical Informatics"), having then a total of 8 different themes.

In 2002, the only General Informatics theme was replaced by another Medical Informatics theme ("Signal Processing").

The number of practical Medical Informatics works presented by the students in 2001 was 15, representing approximately 90 students. In 2002, the number of MI works was extended to 21, representing approximately 121 students. The teachers supervising groups has grown from 2 in 2001 to 4 in 2002, lowering the average of 7.5 groups per supervisor to 5.25 groups per supervisor.

These works, have examined topics like "Telemedicine", "Prescription systems and Drugs databases", "Electronic Patient Records", "Security on Medical Informatics", "Classification and coding", "Clinical Decision Support Systems", "Medical informatics and Education", and "Health professionals use of Computers and Internet". Students were encouraged to give a national perspective on these subjects. 
Some of the practical works require interviews with health professionals. The lists containing such health professionals are difficult to obtain and very frequently are not updated. Students usually report poor availability from the selected population to answer questionnaires.

These works are annually submitted to the Portuguese National Association of Medical Informatics APIM for publication on their site (http://apim.med.up.pt). They are also available in the faculty intranet (http://intro.med.up.pt), for teacher and student consultation.

Discussion: In this four-year evolution of Medical Informatics teaching, the subjects covered have grown three times (from three to nine). This development, we hope, will represent a better understanding of important topics regarding medical information systems. Nevertheless, this constant adaptation of curricula obliges a lot of work by the teaching staff to prepare pedagogical material for the next year.

Proper group supervising is an essential task to achieve good quality works, but it is very time consuming, so a low ration of groups per supervisor is crucial.

To reduce supervisors' efforts, it is suggested that the practical works be distributed for supervisors according to their research interests.

Medical informatics will certainly evolve quickly in the next years, so we intend to continue our curricula adaptation. We aim at producing education and training in medical informatics that is extensive, actual, relevant and captivating. This objective implies that teachers must be up-to-date in technology news, teaching methods and information systems used in a working environment.

\section{References}

[1] IMIA WG1 (Health and Medical Informatics Education). IMIA Recommendations on Education in Health and Medical Informatics. http://www.imia.org/wg1/. accessed 15 September 2003.

[2] J.H. Van Bemmel and M.A. Musen, eds, Handbook of Medical Informatics, Springer Verlag, 1997.

[25]

Consent and Access Issues in the Development of a Privacy/Security Framework for Exchange of Health Information in New Zealand

Pinsara A.B. Galpottage and Anthony C. Norris

University of Auckland, Auckland, New Zealand

The Conference Paper: This paper describes a New Zealand project to provide guidelines and principles for patient consent within a national privacy, authentication and security framework for the electronic access and transmission of healthcare information based on Internet and intranet technologies. The paper identifies the consumerist forces that are driving this development and the government's response. It outlines New Zealand's current and future use of the Internet and dedicated intranet for electronic healthcare transactions and explains how the framework will build upon this technology and the country's existing privacy legislation. The paper contains a brief survey of the New Zealand healthcare system for those who may not be familiar with it and places the developments in an international context. The following sections indicate the topics for discussion.

Patient Consumerism and Health Informatics: New Zealand is reflecting global trends by viewing patients seeking care from both public and private providers as consumers of healthcare services. The recent national health informatics strategy, the WAVE report [1], makes explicit reference to patients as consumers of healthcare services and cites 'increasing consumer demand' when recording the need for 
additional services. The strategy further recommends the use of 'consumer focus groups' and 'consumer advisory boards' to determine the needs and interests of patients in an electronic environment. It also anticipates that identifiers in electronic health databases will be increasingly termed as 'consumer identifiers' and 'provider identifiers. The idea of patient consumerism is similarly prevalent in patient groups such as the 'Mental Health Consumers Union Inc' and in various Maori enabling projects.

Internet Access of Health Information: The New Zealand Ministry of Health (MoH) plans to communicate future health transactions via a dedicated network, currently being implemented as the "Health Intranet". The intranet will be used for highly secure communication but technologies under development to enhance Internet security will provide viable options for healthcare transactions requiring a lower degree of security. These alternatives will allow patient consent to be communicated over the Internet through the use of encryption, digital signatures, and public key infrastructure (PKI) solutions [2]. The impact of $\mathrm{MoH}$ research [3] on broadband Internet access will also be useful in exploring options to communicate patient consent.

The PAS Project: The MoH is combining these different elements into an integrated Privacy, Authentication and Security (PAS) framework. The purpose of PAS is to ensure that the participants in a healthcare transaction are authenticated (they can be identified), their actions cannot be repudiated (proof of action is available), any transaction is secure (cannot be intercepted), and meets privacy standards (personal information is not disclosed inappropriately) [4]. The PAS framework will build upon New Zealand's longstanding and highly regarded privacy legislation [5] which commences with the 1956 Health Act, continues with more general legislation such as the Official Information Act 1982, and draws upon recent changes in technology and practice encapsulated in the Health Privacy (1994/6) and Health Network (2003) Codes together with international (ISO 17799) and national standards for information security management [6]. Once it is consistent and reliable, the PAS framework will be adopted by the New Zealand Health Information Standards Organisation and disseminated to achieve uniform privacy and security standards appropriate to various healthcare organisations, including patient consent management processes.

Databases of Electronic Health Information: The need for privacy and security in healthcare transactions is especially important to control the access and use of national databases that store patient information. New Zealand has several of these databases including the National Minimum Data Set, Medical Warning Systems, Mental Health Information Collection, and the Health Practitioner Index (under development). The MoH administers most of these databases through the New Zealand Health Information Service. The most central of these databases is the National Health Index of patient identifiers. These identifiers link to the other databases to access and share health-related information. The $\mathrm{NHI}$ is only released in encrypted format to prevent identification of confidential data and the PAS project will define the circumstances of its use and the linked databases.

Patient Consent Issues: A major issue arising from obtaining patient consent is the effect on the physician-patient relationship [7]. Research into informed consent covers many different medical issues $[8,9]$ including amongst others mental healthcare, emergency and intensive care, surgery, postmortem/organ donation, HIV patients, and substance abuse. Another important group of issues arises with consent for medical research, especially for clinical trials involving human subjects. In New Zealand consent for treatment is not required as patients are expected to know the purposes for which their information will be used. Consent is, however, needed for elective treatment, research and for subsequent release of identifiable information to third parties including a patient's family and other interested parties [10].

International Practices for Electronic Consent: There is no electronic consent management process in New Zealand and there are no proposals to build a system to manage this vital aspect of patient privacy. 
In contrast, Australia has several projects that seek to manage patient consent within the HealthConnect electronic health environment. Australia's Gatekeeper system of PKI security solutions for public information transactions will be used to ensure the security of electronic consent communicated between various health care providers [2]. In the UK and Canada, the legislative and regulatory environment requires the use of explicit patient consent before using and/or sharing patient-identifiable information in their healthcare environments [11]. In the USA, the requirement for mandatory consent under the Health Insurance Portability and Accountability Act 1996 [12], has been scrapped by the current administration, requiring only notice of privacy practices of the health care provider to the patient. The paper will compare these different approaches and their implications for New Zealand.

\title{
References
}

[1] The Wave Advisory Board, From Strategy to Reality: the WAVE Project, New Zealand Ministry of Health, October 2001.

[2] Gatekeeper: A strategy for public key technology use in the Government, Australia Office of Government Information Technology, 1998.

[3] Provincial Broadband Extension (PROBE) Project Overview, New Zealand Ministry of Education, 18 July 2003.

[4] PAS Project Management Plan (PMP) v1.4, Genesis Consulting New Zealand, 13 May 2003.

[5] New Zealand Privacy Act 1993, New Zealand Parliamentary Counsel Office, 1 July 1993.

[6] Security In Government Sector (SIGS), New Zealand Interdepartmental Committee on Security.

[7] S. Buetow, J. Cantrill and B. Sibbald, Risk communication in the patient-health professional relationship, Health Care Analysis 6(3) (September 1998), 261-268.

[8] J. Sugarman, D.C. McCrory and R.C. Hubal, Getting meaningful informed consent from older adults: a structured literature review of empirical research, Journal of the American Geriatrics Society 46(4) (April 1998), 517-524.

[9] M.B. Kapp, Evidence-based medicine and informed consent, Academic Medicine 77(12 Pt 1) (December 2002), 11991200.

[10] S. Dovey and M. Tilyard, Data protection and patients' consent: New Zealand model of consent offers solution, British Medical Journal 322(7285) (3 March 2001), 550.

[11] H.A. Vohra, J. Ledsham, H. Vohra and R.L. Patel, Issues concerning consent in patients undergoing cardiac surgery - the need for patient-directed improvements: a UK perspective, Cardiovascular Surgery 11(1) (February 2003), 64-69.

[12] Public Law 104-191, Health Insurance Portability and Accountability Act 1996, 104th United States Congress, 21 August 1996.

\section{[26]}

\section{Empowering patients and professionals by moving electronic health learning into other media to increase access, relevance and equity}

\author{
Bob Gann and Bette Jane Baldwin \\ NHSDirectOnline, Hedge End, England
}

The advantage of supporting the resourceful health service user with a wide range of formats in which to access and use information is clearly recognised. NHSDirectOnline has been at the forefront of this activity in the UK working collaboratively with NHSDirect to provide information and support to empower patients and citizens to resolve health problems and to improve personal health and wellbeing. NHSDirectOnline has done so in a range of media including telephone call centres, self help guides,kiosks and the NHSDirectOnline website. The NHSDirectOnline website provides three key resources to support and provide health information: an Online Health Encyclopaedia, a Questions and Answers Self Help Guide and an Online Enquiry Service. In addition the Website provides links to an increasingly wide range of health information resources. 
The Website is shortly to be expanded to include a user interface resource, "Contact Us" which will provide online opportunities for users to get help, advice, support to use the website, make comments, suggestions and complaints and also join the new Online Readers Panel which will provide online lay interaction with the Editorial Team. Other new developments are now underway which are taking the learning from the NHSDirectOnline Website on and into other media including, the new Digital TV NHSDirectOnline Development, the new Kiosk programme and the electronic My NHS Healthspace development. These developments will be joining the NHSDirectOnline stable of media formats to support health service users. These developments seek to respond to the well understood need for different courses for different horses in terms of how users wish to access and use information resources.

The movement into other media also ensure that there is continued expansion of the target audiences who can access health information in a form and media which is relevant and accessible to their needs. This paper and its presentation will give an overview of the advantages of multimedia support to the resourceful patient, present the evidence behind the development of the NHSDirectOnline multimedia resources as well as the ongoing research and evaluation activities. It will present research data from piloting activities which has demonstrated the improvements in targetting difficult to reach audiences and the fundamental need to involve the user in the development of these new media formats. It will outline the manner in which the learning from the creation and day to day running of a national health website has been used to influence and inform these new health media developments and discuss the challenges involved.

\title{
[27]
}

\section{A descriptive analysis of strategies in Spain for internet biomedical content evaluation}

\author{
S. García, E. Montesinos, V. Baujard and C. Boyer \\ Health On the Net Foundation, Geneva University Hospital, Switzerland
}

Learning objective: After this presentation participants should be able to understand the following points. How the coexistence of several quality seals certifying one website can influence internet users, health professionals as well as general public. To determine if the existence of a growing number of initiatives regarding health content quality is beneficial. And to determine if a closer interaction amongst these initiatives is needed.

Introduction and goals: The number of health related websites is rapidly growing, as well as the number of internet users in search of health information. The number of initiatives aimed at improving the quality of the health information offered are growing as well. The health information offered is very diverse, trusted medical information, reviewed by health professionals can be found, as well as information with no scientific support or background. This has lead to the development of control and evaluation tools.

The goal of these initiatives is to guide internet users in there search of health information. This guidance is done by determining if a website follows certain quality criteria regarding authorship, website funding and the existence of conflict of interest.

Different Spanish speaking initiatives can be found. By means of a health website certification process they guide the internet user.

We will study each one of them, comparing the methods they use and evaluate the coexistence of the different quality seals in a website.

Study design: 
- First phase: Description and observation of Spanish projects for the certification of health information websites, by independent research in three generic search engines, five specialized search engines, Medline database and Spanish Medical Index. Keywords used: "internet, quality, information, ethic code, certification". The HON Foundation is excluded as it is used as the "gold standard", this initiative has the largest number of certified Spanish speaking websites. The initiatives are selected after evaluating authorship, institutional support, existence of a quality seal, how authorization is given to display the quality seal, and a listing of the websites certified. Each initiative is compared with the quality criteria established by the Health on the Net Foundation and the European Commission.

- Second phase: Independent observation by two researchers of the homepage of all the websites listed by each initiative. In each homepage the presence of one or more quality seals has been evaluated. If the seal was not found in the homepage, it was found in sections such as "credits, certifications". For the HONcode quality seal, the certification process status page was reviewed as well.

Regarding statistical analysis, a descriptive listing of the data was used.

Results: From April to June 2003, besides HON, 9 health specific initiatives were identified. Each one provided a description of the criteria used for evaluation, certification and authorization to display there quality seal. The list of the initiatives studied is:

1. http://wma.comb.es/cat/llistat.php (seal name: Web Médica Acreditada; $n=235$ ).

2. http://www.pwmc.org/pwmc.php3 (seal name: Web Médica de Calidad; $n=62$ ).

3. http://www.hipocampo.org/ (seal name: Web Recomendada; $n=26$ ).

4. http://www.webmastersanitarios.org/Asociadas/asociadas.asp (seal name: SWS Calidad Biomedicina; $n=98$ ).

5. http://www.aurasalud.com/ (seal name: Web de Interés Divulgativo; $n=42$ ).

6. http://www.portalesmedicos.com/premio/medicina/ganadores.htm (seal name: Premios Web; $n=45)$.

http://www.portalesmedicos.com/premio/medicina/2001.htm

http://www.portalesmedicos.com/premio/medicina/2000.htm

7. http://www.seei.es/certificados/paginascertificadas.htm (seal name: Certificado de calidad de páginas electrónicas; $n=40)$.

8. http://aemir.org/ (seal name: Web de Utilidad en Formación Médica Continuada $n=52$ ).

One initiative was disregarded (http://www.cristosalud.com) as it was unavailable at the time of this study.

The number of websites reviewed by each initiative ranges from 26 to 233 . The total number of websites reviewed by the 8 initiative is $600(n=600)$. 174 websites $(29 \%)$ were using more than one quality seal and $35(6 \%)$ were websites not found by the server. The number of websites displaying the HONcode (Health on the Net quality seal) was $215(36 \%)$, of which 72 are under revision process.

The variability found in the seal being displayed by the website was high, only 53\% (range 23\%-96\%) of the websites certified display the quality seal. $60 \%$ of the sites display more than one quality seals. In some cases 6 quality seals were found in the website.

The criteria used by each initiative is based on the quality Principles established by the Health on the Net Foundation and the European Commission. Some also include an evaluation of aspects related with the design, the accessibility, the existence of a sitemap, the differentiation of content for the general public and medical professionals, the use of references to evidence based medicine and practice guidelines and the use of plain language. All of the initiative are free of charge for the website. 
Conclusions: It has been noticed that most of the initiatives that guide the health internet users, by certifying health websites with a quality seal do not remain active for a long period of time.

The existence of multiples quality seals in one website does not indicate a higher quality of the website, regarding the principles of the different initiatives evaluated.

Users, and mostly patients, are not aware of all the quality initiatives existing, and they are not aware of the importance of looking for quality seals when doing there information search. A proposal to develop a platform, sponsored by each country government, with the collaboration of public institutions, professional associations, scientific societies and user associations, unifying the experience of each quality initiative should be taken into account, in order to improve the awareness of users and patients and avoid the confusion that comes from the existence of multiple quality seals. This awareness would keep the poor quality and fake medical information from the user-patient, and would avoid the consequences of misleading medical information. The European Union is studying the development of a joint action among its members to establish a quality seal for health websites. Initiatives such as MedCIRCLE have this objective.

Keywords: Internet, quality, information, health, ethic code, certification.

Conflict of interests: The authors have not received any external funding for this study. They are consulters with the Health on the Net Foundation.

\title{
References
}

[1] J.F. Ávila, B.E. Portillo and J.M. Pajares, Calidad en la información biomédica existente en Internet, Aten Primaria 28(10) (2001), 674-679.

[2] G. Eysenbach, J. Powell, O. Kuss and E.R. Sa, Empirical studies assessing the quality of health information for consumers on the World Wide. A systematic review, JAMA 287 (2002), 2691-2700.

[3] S. Fox and D. Fallows, Internet Health Resources: Health searches and email have become more commonplace, but there is room for improvement in searches and overall Internet access. Pew Internet \& American Life Project. 2003. Available in: http://www.pewinternet.org/reports/.

[4] A. Gagliardi and A. Jadad, Examination of instruments used to rate quality of health information on the internet: chronicle of a voyage with an unclear destination, BMJ 324 (2002), 569-573.

[5] M.A. Mayer, Acreditar webs de contenido sanitario: ¿una necesidad imposible? Med Clin (Barc) 116 (2001), $496-497$.

[6] G.P. Purcell, P. Wilson and T. Delamothe, The quality of health information on the internet, BMJ 324 (2002), 557-558.

[7] R. Smith, Almost no evidence exists that the internet harms health, BMJ 323 (2001), 651.

\section{[28]}

\section{WRAPIN (Worldwide online Reliable Advice to Patients and Individuals)}

\author{
A. Gaudinat and C. Boyer \\ The Health On the Net Foundation, Switzerland, www.hon.ch
}

Keywords: Biomedical informatics, eHealth networks and architectures, health professionals knowledge, public health, health promotion, information to citizens, security and privacy.

Objectives: WRAPIN stands for Worldwide online Reliable Advice to Patients and Individuals. WRAPIN, a two year European project (IST-2001-33260) helps solve two major problems faced by Internet citizens: Finding information among a large number of potentially useful documents; Evaluating the relevance of an online medical document. Despite the improved access to information made possible by the Internet, it remains extremely difficult to judge the trustworthiness or relevance of online content. All categories of users, professional and non-professional, face this problem, which can be of critical 
importance in the case of health or medical information. Search engines, some general, some specialized, are used by all categories of users to find information but the ever-increasing number of online documents means that queries must be ever more precise.

WRAPIN involves six partners, leaders in their respective fields; HON, THALES Information Systems, the Medical Informatics Division of the University Hospitals of Geneva, Nice Computing, LERTIM of the Faculty of Medicine of the Hôpital de la Timone and the Ministry of Health and social actions (MISS).

WRAPIN is an ambitious project aiming to support the online citizen with a practical, convenient solution to the problem of large numbers of documents of uncertain quality. WRAPIN will offer a modular platform to interconnect the most trustworthy and complete information sources. To date, the following databases have been integrated in WRAPIN:

- MEDLINE, containing scientific medical articles from the 1960's through the present and constantly updated;

- Clinical Trials (clinicaltrials.org);

- OESO (new electronic database prepared by WRAPIN for the World Organization for Special Studies on Diseases of Oesophagus;

- Urofrance (French urology database);

- MedHunt (medical search engine) and

- the HON Code of Conduct (http://www.hon.ch/Conduct.html) accredited Web sites.

- the HONnews daily news from HON and HealthDay

- Drug Information from the Drug and Food Administration (FDA)

The resulting search tool will provide citizens with a wide range of trustworthy medical and health content.

Results: The WRAPIN project makes use of new developments in the mapping of medical terms, indexing and search technologies, and natural language processing tools. WRAPIN has also incorporated and stimulated progress on ongoing projects, such as MARVIN (Multi-Agent Retrieval Vagabond on Information Network, HON's medical web spider), HONselect (the 'categorizer' which currently provides a directory of health/medical resources), ARIANE (the 'query conceptualizer' from LERTIM), and a 12-language spelling corrector. WRAPIN will use these technologies to help in query formulation, leading to accurate search results.

WRAPIN will also provide an entirely new facility enabling the comparison of health/medical documents in any format (HTML, PDF, etc.) or length with this interconnected knowledge base, to discover if the information exists in the published literature and provide a summary conclusion of the ideas contained. It will help to determine the reliability of documents by checking the ideas contained against established benchmarks, and enable users to determine the relevance of a given document from a page of search results. This exciting new tool is an important step toward the certification of quality online information.

Acknowledgements:

The WRAPIN (Worldwide online Reliable Advice to Patients and Individuals) project, IST-200133260, is supported by the European Commission and the "Office Fédéral de l'Education et la Science" (OFES, Switzerland).

The authors wish to thank all the WRAPIN members, with their precious contribution in this ambitious project; HUG - SIM Service of Medical Informatics - University Hospitals of Geneva [11], LERTIM Laboratoire d'Enseignement et de Recherche sur le Traitement de l'information Médical [12], MISS Mission pour l'informatisation du système de santé [13], NICE Computing [14], THALES Information Systems [15] and XRPartner [16]. 


\title{
References
}

[1] Clinical Trials: www.clinicaltrials.gov.

[2] Drug FDA: www.fda.gov/cder/drug/default.htm.

[3] P. Ruch and A. Gaudinat, Combining regular variation and nearest neighbors for efficient mapping to indexing terms. Romand 2002, Frascati. The 2nd Workshop on Robust Methods in Analysis of Naturallanguage Data, 72-79, July 2002.

[4] A. Gaudinat and C. Boyer, Automatic Extraction of MeSH terms from MEDLINEs Abstracts. Workshop on Natural Language Processing in Biomedical Applications, NLPBA2002: 53-57.

[5] A. Gaudinat, M. Joubert, PhD, S. Aymard, L. Falco, C. Boyer and M. Fieschi, Indexation et recherche d'information biomédicale: une nouvelle approche intégrant les sources de connaissance UMLS à l'extraction de termes MeSH. Xemes JOURNEES FRANCOPHONES D'INFORMATIQUE MEDICALE 2003.

[6] HealthDay: www.healthday.com.

[7] MedLine: www.ncbi.nlm.nih.gov/PubMed.

[8] Oeso, World Organisation for Specialized Studies on Diseases of the Esophagus: www.oeso.org.

[9] UroFrance, le site de l'Urologie française: www.urofrance.org.

[10] WRAPIN: www.wrapin.org.

[11] Service d'Informatique médicale: http://www.sim.hcuge.ch/.

[12] LERTIM: http://cybertim.timone.univ-mrs.fr/cybertim.

[13] MISS: http://www.emploi-solidarite.gouv.fr.

[14] Nice Computing: http://www.nicecomputing.ch.

[15] THALES IS: http://www.thalesgroup.com.

[16] XRPartner: http://www.mediqual.net/.

\section{[29]}

\section{Patient Education and Service Kiosk: Health Education at the Point-of-Care}

\author{
Leonard Goldschmidt and Gregory Goodrich \\ Department of Veterans Affairs Palo Alto Health Care System, Livermore, USA
}

Objectives: We sought to create an easy to use, health information resource for patients at the point of care, the patient waiting area. An important innovation is the ability for the patient, after viewing multimedia, validated content, to print vouchers redeemable for suitable clinical care, including diabetic eye and foot examinations, and flu and pneumonia shots. The overall aim of the kiosk, besides allowing patients to take control of their health, is to increase compliance with selected recommended guidelines designed to improve health. Study Design: The study is prospective and non-randomized. Methods: Vouchers redeemable for clinical services were collected and triaged. Statistics on clinical outcomes are provided through our computerized medical record database. In addition, results of a patient survey, containing a number of repeated internal validation questions, are presented. The study protocol went through the institutional review boards of Stanford University Medical Center and the Department of Veterans Affairs Palo Alto Health Care System, and privacy and research statements are included in the opening page. Results: Seven hundred and thirty nine users participated in the questionnaire over a one-year period, while a total of 7,460 user sessions were recorded. Seventy six percent were first time users, and $72 \%$ were using the kiosk at their own desire, rather than the recommendation of their doctors or staff. Eighty-six percent of respondents thought that the kiosk was well located, and 84-89\% (repeat internal validation questions) said the instructions for the kiosk were easy to follow. Ninetyfour percent of respondents stated that the information on diabetes was helpful to them. Over $72 \%$ of respondents found some or all of the information they were looking for on the kiosk, and $88 \%$ said they would use the kiosk in the future. Seventy five percent of people said that the information on the kiosk was helpful in understanding their health, and $90 \%$ of people said the clinic had more to offer because of the patient education kiosk. Additionally, $86 \%$ said it was very likely (60\%) or somewhat 
likely (26\%) that what they learned at the kiosk would help them follow a health provider's advice. Most importantly, in the year following introduction of the kiosk intervention, a $14 \%$ increase in the number of patients receiving flu vaccinations was recorded, compared to the prior year. Conclusions: We have created a patient education and service kiosk, providing a range of health content, spoken in English or Spanish. It includes validated information on diabetes, cancer, disabilities, hypertension, cholesterol, back pain, heart disease, and traveler's tips for staying healthy, using tested principles of health education. Also, approximately twenty selected English and Spanish language health-related web sites are accessible through the touch-screen kiosk, at high broadband speed, in a seamless manner. This provides access for patients who might not otherwise find validated medical information in this manner. It also gives patients an opportunity to better utilize free time around or between appointments. Databases of diseases, medications, and herbal supplements have been licensed and are available. Most people found the kiosk easy to use and are likely to use it again. By allowing people to print out and take home accurate detailed information for later review, it promotes patients' retaining and utilizing important health related information. Most survey respondents found the information useful and feel the clinic has more to offer because of the kiosk. It is an opportunity for people to gain the maximum amount of information during each clinic visit. Most significantly, patients felt they would be more likely to follow health care providers advice because of the kiosk $(85 \%+)$, and there was a recorded $14 \%$ increase in the number of flu shots dispensed after the introduction of the kiosk, although there may be other unknown factors that influenced this finding. The kiosk has the potential to make every patient encounter a chance to favorably influence clinical outcome.

[30]

\section{P.A.I.N. Quality: Electronic documentation for routine use in quality assurance and health care management}

Klaus Ernst Groth ${ }^{\mathrm{a}}$, Frank Heinzen ${ }^{\mathrm{a}}$ and Marc Kurepkat ${ }^{\mathrm{b}}$

${ }^{a}$ Grunenthal GmbH, Germany

${ }^{\mathrm{b}}$ Institut fur Gesundheits- und Sozialforschung, Aachen, Germany

Chronic pain is a major European healthcare problem. Appropriate pain management is still not available for the majority of patients. Concerted action could lead to improvement, but it still has to be developed. Objective of the international P.A.I.N. initiative is to elaborate feasible and outcomeorientated concepts to implement a continuous quality improvement process in routine clinical practice. P.A.I.N. methodology is based on a modified Plan-Do-Check-Act (Total Quality Management) circle. Evidence-based guidelines are often ignored and felt to be not relevant for routine practice. As a consequence experience-based educated guesses have been made to describe and compare the ideal and real conditions, based on measurable indicators, having threshold values, usable to indicate designated measures, suitable to realise desired results. These results will be re-evaluated, which could lead to changes in practice. Prerequisite is an instrument for a valid and standardised documentation: P.A.I.N. Quality is a new computerised documentation programme for routine use. It offers a continuous clinical assessment by comparing the documented findings with pre-defined standards. Evaluation of patients risk factors, combined with a alert function, a scenario-driven clinical guidance and a visual monitoring of case-related efficiency and effectiveness. To identify best practice statistical key data from equivalent medical service providers are compared in an anonymous external ranking (Benchmark). By publishing this reality-based data it is intended to produce a learning environment, able to guarantee a continuous quality improvement. Additionally to support continuos improvement processes the programme also 
offers the opportunity to be applied in more quality-assurance-orientated projects like the German Disease Management Programmes (DMP). In this context P.A.I.N. Quality presently is being applied in a practitioners network which is involved in DMP Back Pain. The P.A.I.N. Quality programme used in this project is running using the internet. Furthermore it provides current electronic feedback to the involved practitioners with instruments like reminders (prospective) and reports (retrospective). The project can be regarded as a pilot scheme in Online-Disease-Management in Germany and will be continued until September 2004.

\title{
[31]
}

Internet-based patient education program for asthmatic children and adolescents: Acceptance and cost-benefit analysis

\author{
Michael Horn \\ Forum-Telemedizin GmbH, Ramsau, Germany
}

Objectives: Asthma bronchiale is the most common chronic disease in German children and adolescents and has a prevalence of over 10\%. The conventional structure of Asthma education, by consensus, follows Arbeitsgemeinschaft Asthmaschulung im Kindes- und Jugendalter and has proven to be successful for improving health. The study was to show that if the tailor-made Internet education tool, Forum-Telemedicine (FTM), was used as a follow-up for six months, patients would show a continued improvement of the management of their condition through patient education and empowerment. This would result in a reduction in cost of medication, less visits to GP / Hospital with acute symptoms and less time off work / school. FTM is based on the traditional education given to the patient when they are initially diagnosed but delivers an additional follow-up in a modern and new way. Its internet-platform contains an adventure game with asthma-related situations, a medical area with online peak-flow-monitoring and therapy plans, online chats with asthma-experts, an interactive quiz and a community area. It is not intended to replace the traditional education by an internet web page but to be used as a virtual patient lead continuation of the learning process.

Methods: An acceptance study with 50 patients as well as a prospective cost-benefit analysis alongside a non-randomised trial with 438 patients in 34 study centres with two intervention groups and one control group were conducted. Within the acceptance study patients participated in FTM following the traditional educational program whereas within the cost-benefit analysis both intervention groups received traditional training, but only one group the FTM-follow up. We collected data at the first visit as well as after six and twelve months.

Results: The results underline that this specific internet-based asthma education tool is widely accepted by patients resulting in a favourable benefit-cost ratio.

Conclusions: FTM as an add-on to traditional asthma education is an adequate channel for efforts to improve asthma self-management on a long-term basis reducing costs at the same time.

[32]

Suicide and the Internet

Robert C. Hsiung ${ }^{\mathrm{a}}$, Azy Barak ${ }^{\mathrm{b}}$ and Denise Silber ${ }^{\mathrm{c}}$

${ }^{a}$ University of Chicago, Chicago, USA

${ }^{\mathrm{b}}$ University of Haifa, Israel

${ }^{\mathrm{c}}$ Basil Strategies, France 
In this workshop, we explore the topic of suicide and the Internet from three perspectives. First, we report a suicide in an online peer support group in the United States. Next, we present an online suicide prevention service in Israel. Finally, we address the ethical responsibilities of the administrators of sites like these. This is, to our knowledge, the first report of a suicide in an online group. The group was a peer support message board focused on mental health and managed by a mental health professional. The reactions of the group members to the suicide was as varied as might have been expected in "real life" and included the wish for more information as well as outright disbelief; feelings of sadness, helplessness, anger, and loss; physical symptoms; remembrances of the deceased member; self-reflection; and support for each other. There were questions about the boundary between "e-life" and real life. Should group members use her real name? Go to her funeral? The board appeared to function as an effective "holding environment." No group members reported injuring themselves - or were reported to have suicided in response. Administrative issues included notifying the institutional review board involved (the group was at the time considered a research study), ensuring that additional resources were available for those coping with crisis, and establishing some sort of memorial. A concern regarding the memorial was suicide "contagion." In the end, it simply linked to selected posts: remembrances, songs and poems, posts written to her *after* her suicide, and posts of her own, including ones about a previous suicide attempt and on her philosophy of suicide. The memorial area may function as a virtual cemetery for the virtual community. Having the administrator establish it may in part have served to reassure surviving group members that he cared about the deceased member - and, by extension, about them. SAHAR an original, free Israeli online service - set itself the goal of arranging an online environment in which Web surfers in distress would not encounter confusion and panic, on the one hand, or incitement and recipes for suicide, on the other. Rather, they would get in touch with trained helpers who would listen to them; offer support, advice, and referral; and, if need be, act to save their lives. All of this is now offered through a free, easily accessible, exclusively online service. SAHAR offers to surfers a comprehensive, content-rich, and continuously updated Web site; individual support through e-mail or chat; and group support through several forums. Over close to three years of operation, over 300,000 people have used the various help channels provided by SAHAR. Approximately 25,000 personal contacts have been made with people in distress, over $50 \%$ of whom were clearly suicidal. In addition, over 500 people in severe distress have used and found relief in using the online support groups. In all cases, suicide was successfully prevented by providing emotional support, significant and badly needed information, and referrals to professionals - or by last-minute rescue operations launched in cooperation with relevant authorities. Not a single suicide committed by a SAHAR referral has been identified so far. The enormous success of SAHAR provides additional support for the idea of using the Internet as a valid channel for psychological help. Online peer support groups and suicide prevention services present, like many innovations, ethical questions. Users need to understand the potential risks and benefits of using such sites and the alternatives to doing so. In particular, the sites should disclose to users the potential risks to their privacy posed by the electronic communication and storage of information and the safeguards taken to prevent unauthorized access to their personal data. Limits to their confidentiality, for example in situations of danger to themselves or others, should be explained. Electronic communication may make misunderstandings more likely. The sites should assure that the services they provide are of high quality. Individuals providing them should be competent to do so. It should be clear what procedures that the sites will, and that users should, follow in an emergency. The ability of sites to rescue anonymous users located far away may be limited. The evidence base for such resources needs to be developed. If the procedures are experimental, users should be so informed. E-health ethics guidelines may help the developers and administrators of such sites to design and manage them more effectively and assist potential users in making more informed decisions regarding their use. 
[33]

Universal accessibility to personal medical data: Issues for future development of health services

Jorge Martinez de Hurtado

Fundacion Hospital Alcorcon, Alcordon, Spain

Objectives: Understand the benefits of an Electronic Health record (EHR) distributed through the Internet or its related technologies, so that it is readibly accesible independently of location. However, important ethical, legal and security issues must be solved not only from the technology point of view, but also from that of the different actors of health care systems.

Abstract: Medicine has been considered for many authors one of the last activity sectors in which paper based transactions take place. Nonetheless, surprise arises when one learns that health organizations are information intensive and information dependent in their daily routines. Reducing medical errors and improving accessibility to medical information is used by many as the main reasons to computerize medical activities. Predictions from years ago stated that, by now, EHRs would be fully deployed in healthcare. However, up to date, only $5-10 \%$ of doctors fully use this tool. Advantages as multipoint access to clinical record, introduction of relevant clinical information by provider/patient, and personalization of general medical advice, must be ensured by good design, using preferably open standards that include concepts as integrity, interoperability, accessibility, security, traceability and flexibility. Using the Internet (or related technologies) to transmit and exchange information, allows to use it at the point of care. Several barriers to the deployment of an efficient tool arise, related to identification of users, security requirements, contents, format, language and cost. Deployment of an Internet distributed, secure and transversal EHR, with partial control by the patient, can be simple and practical enough for it to show all its benefit. The components of such tool must include, at least, a connectivity engine, a unique identification system, master patient index, electronic document manager, workflow processor and image management capability. According to recent surveys, the reasons for health care systems to use EHR are the following: Need to share comparable patient data among different sites within a multi-entity healthcare delivery system $75.7 \%$; Need to improve clinical documentation to support appropriate billing service levels $75.3 \%$; Contain or reduced healthcare delivery costs $66.3 \%$; Need to establish a more efficient and effective information infrastructure as a competitive advantage $64.3 \%$; Need to meet the requirements of legal, regulatory, or accreditation standards $60.4 \%$; Need to manage capitation contracts 21.8\%; Other reasons 3.5\%; Source: Medical Records Institute. If we just analyse the clinical issues towards the use of EHR Systems, the results are: Improve the ability to share patient record information among healthcare practitioners and professionals within the enterprise $90 \%$; Improve quality of care $85.3 \%$; Improve clinical processes or workflow efficiency $83.6 \%$; Improve clinical data capture 82.4\%; Reduce medical errors 81.7\%; Provide access to patient records at remote locations 70.7\%; Facilitate clinical decision support 70\%; Improve employee/physician satisfaction 63\%; Improve patient satisfaction 60.4\%; Improve efficiency via pre-visit health assessments and post-visit patient education 40.2\%; Support and integrate patient healthcare information from Web-based personal health records 30.4\%; Other 0.3\%; Source: Medical Records Institute. EHR systems are, in fact, quite complex. Considering every potential option, ten dimensions can be analysed: 1 . Information capture; 2. Information representation; 3. Data flow; 4. Clinical practice; 5. Decision making support; 6. Trust and security; 7. Activity indicators; 8. Interoperability; 9. Quality control; 10. Content selection. In a too high percentage of cases, nowadays doctors lack necessary and precise information at the point of care, so that unnecessary ancillary diagnostic methods are blindly repeated, and previously detected 
conditions are unknowingly ignored. A policy of integration of EHR systems and the Internet can solve many of the dilemmas and challenges to which health care systems are faced during the 21 st century.

\section{[34]}

\section{What Are They Looking For? A Survey of Searches on KidsHealth}

Neil Izenberg, Elana Ben-Joseph and P.J. Gorenc

Nemours Center for Childrens Health Media, Wilmington, USA

The Web has made it easy for families to have access to vast amounts of health information on demand, 24 hours a day, privately, and without the involvement of any health care provider. Online searches are increasingly being used as the primary source of health information for many family members. But, exactly what sort of information is being sought? Our search of the medical literature has revealed no reports to date regarding the specific nature of consumer Web searches regarding health information, despite the potential utility of such data.

To better understand which children's health and development issues various family members are looking for, researchers at the Nemours Center for Children's Health Media performed a study of search terms used to find information at KidsHealth.org.

KidsHealth, with typically more than 120,000 visits each weekday, is one of the most viewed sites with consumer-oriented information focusing on children's health. Topic areas cover a wide range of issues, including medical problems, growth, nutrition, physical and emotional development, behaviors, and family function - from pregnancy through adolescence.

On the Web since 1995, KidsHealth creates and maintains thousands of physician-reviewed articles, graphics, animations, and other features concerning children's health for three separate audiences: Parents, Kids (ages approximately 8-11), and Teens (ages approximately 13-18). Each area of the site has its own age-appropriate graphic style, language level, and topics tailored for that specific audience. The site is created by the nonprofit Nemours Foundation's Center for Children's Health Media, a pediatrician-led group that creates educational online, print, and video material for families.

Each week, visitors to KidsHealth conduct approximately 100,000 searches within the site looking for answers to their questions - and hundreds of thousands more on search sites such as Google and Yahoo! that lead directly (are hyperlinked) to articles on KidsHealth. Analyzing these searches can give insight into children's health information needs regarding of Web users - and could help creators of Web content to meet the need of site visitors.

Methodology: We selected a convenient 5-day period (from October 1 through 5, 2003) to examine what topics searchers were looking for on KidsHealth.org. Consistent with its long-standing policy to ensure privacy, KidsHealth does not "cookie" individual visitors. Considerable amounts of user data, including specific terms entered by visitors and page views, however, are collected via web logs at a separate server at KidsHealth dedicated to that purpose. The information collected allows us to see the exact words and phrases used during searches.

During the study period the site received 175,473 total searches from KidsHealth and from the Google and Yahoo! websites, of which 163,214 could be categorized. Of the total number of usable searches, $31 \%$ originated from within KidsHealth and 69\% from Google and Yahoo!.

We took a random sample of 4,000 (or approximately $2.5 \%$ of the 163,214 searches) and analyzed terms by manually placing each in categories reflecting the nature of the search. A few (387) were entered in more than one category. Each search term is associated with one of three areas of content: Parents, Kids, or Teens. Validity of randomization was checked by calculating the total variability of the 
random sample against the population with regard to frequency of occurrence of Parents, Kids, and Teen search terms. Total variability was $2.27 \%$ for onsite searches and $1.5 \%$ for off-site searches.

The searches from within the KidsHealth website originated from three separate sections within the site: Parents, Kids, and Teens. Each of the sections has its own section-specific search box. Searches in the Parents section yield results from all areas of KidsHealth content. Searches in Kids yield results from only within the Kids content, and searches from within Teens yield results only from within the Teens content. Of the searches from within KidsHealth, 51.5\% originated from within the Parents area, $33 \%$ from within the Kids area, and $15.5 \%$ from within the Teens area.

The results revealed that infectious diseases is, by far the most searched topic, for all searches. For Parents, searches about infectious diseases involved $30.6 \%$ of all KidsHealth searches, for Kids $13.9 \%$, and for Teens $12.5 \%$. From off-site (Google and Yahoo), 26.3\% of all searches involved infectious diseases; from on-site at KidsHealth the percentage was $13.8 \%$. That $13.8 \%$ is the highest percentage of any searched topic onsite at KidsHealth.

In general, parents are searchers of condition-related issues: infectious disease, gastrointestinal concerns, and pregnancy. Teens are searchers of issues involving emotions, behaviors, and sex (including pubertal development, reproductive systems and sexually transmitted diseases). Kids are searchers, in general, of how things work. This includes general information such as how the ear works, skeletons, the respiratory system - in short, the mechanics of the body, possibly, in part, for homework assignments. This differentiation is consistent with the development needs of each of these groups.

Comments: Visitors to KidsHealth may find information through a number of methods in addition to the search bars, including use of the site's extensive navigation system visible on every page, including category listings and site "bread crumbs" indicating where on the site a reader is. Limitations to this study include that the searches using the site's search bars may not fully reflect what information all visitors are seeking, indicating instead only those searches involving the search bar. Because KidsHealth does not cookie its visitors for privacy reasons, we do not know in-depth information regarding individuals performing those searches.

Nevertheless, given the immense volume of search-bar carried out on KidsHealth.org each week as well as the volume of children's health-related searches by those using Google or Yahoo! who then land on KidsHealth - evaluating the nature of these searches provides an unstructured way to create a collective profile of user concerns. Analysis of searches on KidsHealth reveals information helpful to those creating health content for families and for those clinicians seeking to know - and address - the issues most concerning families. This short study has revealed substantial amounts of useful data that require additional analysis.

\section{[35]}

\section{The Effects of Medical Information on the Use of the Health System}

Markus Jochem and Susanne Wahling

Techniker Krankenkasse, Hamburg, Germany

The Techniker Krankenkasse (TK) with its 5.4 million insured people is one of the biggest health insurers in Germany. Since 2001 it runs a complex medical information system mainly for its clients, but accessable for all interested users, both via the Internet (including e-mail) and the telephone - with nearly one million users a year. The TK is a non-profit organziation.

The aims of the TK medical information system are to inform the patient about proper ways of preserving or restoring health or to diminish the effects of ill health. As a health insurer, TK obviously 
keeps an eye on the cost situation. The medical information system shall therefore provide the client (and to an growing extent the health professional) with neccessary information, sources and data to avoid inappropriateness and inefficiency, as commonly witnessed in Germany. The given information to the patient, however, does not replace advice of a health professional and a proper medical check-up.

In a new survey to be conducted in August/September 2003, TK will aim at answering questions on the usage of its medical information service. Two key questions are:

After discovering your ill health, at which stage did you use the medical information system (e.g. deciding to use a health professional at all, preparing for a visit to a health professional, reviewing the (shared) decisions made during such a visit)?

Did the information gathered through the medical information system had any effect on your behaviour, your compliance towards prescriptions and your usage of the health system overall?

Results of this survey will be through in October 2003 and will have an impact of the future planning of the TK medical information systems, i.e new steps towards citizen centration and information sharing.

[36]

\section{Improving access to on-line health information for Australian men with prostate cancer}

\section{Craig Jones \\ University of Adelaide North, Daw Park, Australia}

Objectives: To provide needs-based health information to Australian men with prostate cancer: strategies to maximise access

Abstract: Australian men face barriers to accessing information on prostate cancer. Older men have poor access to internet based information, particularly if low income. Rural men have poor access to a specialist Urologist services and often need to travel long distances to access them; prostate cancer diagnosis and treatment is difficult to understand, uncertain and yet too much information can be as great a barrier to understanding as too little. We established a web site which provided high quality needs-based information to Australian men. Evaluation of the first two years of use and comparison with needs- assessment has been published elsewhere. This paper describes strategies which were used in the development of the website to address the above barriers to access. Integrating the promotion of the website with off-line sources of information proved very successful in promoting use of the website. Examples were the annual prostate cancer Call or Click-in where a single national telephone help-line was promoted together with the web address. Callers to the help-line were offered the web-address literature as well as direct answers to their questions. The launch of a consumer guide was also promoted with the web address. The success of this strategy was shown by peaks in website activity at these times and particularly in no referror website referrals ie the URL was typed in directly. This type of promotion increased the proportion of visitors from our target group (Australian men) versus North American visitors. Another strategy to improve access by non-internet using men was to produce a hard copy of the website material: a loose leaf binder with downloads of each of the searchable databases, including the modular information resource. The website itself was designed to provide easy updating of hard copy downloads. This was expensive to produce and not as successful. However it served an educational purpose, so that men with no understanding of the Internet could see the type of information on the website and how it was organised. The hard copy was also used by help-lines and health centres without Internet access. In relation to geographical barriers, we provided a searchable list of Urology services and a map of distribution of Urologists which showed availability restricted to major towns and regional centres.. We addressed the problem of too much confusing information about prostate cancer 
by breaking up information into modules useful at progressive stages in the development of the disease. This part of the site (the Mr PHIP series) became the most widely accessed part of the site. Conclusion Integrating promotion of on-line prostate cancer information with off-line education initiatives assists this target group to access on-line information and is helpful in reducing non-target group traffic. Needs assessment helped us to identify other access barriers such as too much, confusing information and was an important step in setting up an effective website.

\title{
[37]
}

\section{Physician Designed and Directed Internet-based Vardenafil and Sildenafil Prescribing}

\author{
Miles J. Jones ${ }^{\mathrm{a}}$ and Bernard S. Bloom ${ }^{\mathrm{b}}$ \\ ${ }^{a}$ Net Doctor International, USA \\ ${ }^{\mathrm{b}}$ University of Pennsylvania, USA
}

Abstract: The Internet is becoming increasingly important as a way for patients to acquire medical information, as a means for patient-physician communication and most importantly access to medical treatment and therapies. Questions about appropriate use of this new technology have been brought to the fore by the many patients using the Internet to seek medications to treat erectile dysfunction.

Objective: To present a description of a Discuss for the treatment of erectile dysfunction together with data covering more than 25,000 patient encounters.

Design: Retrospective analysis of a large case series, with informal comparison to patients seen in a conventional office based practice setting.

Setting: A medical practice that prescribes sildenafil and vardenafil based on medical and sexual information obtained through a physician designed and directed Internet site. For comparison, we utilized patients from clinics at a Midwestern inner city medical center, patient comments about their office based care and survey information form questionnaires.

Patients: All 26,650 Internet patients seeking vardenafil and/or sildenafil prescriptions between June 14, 1998, and September 10, 2003.

Main Outcome Measures: Completeness of medical record; patient safety as noted by the follow up responses of all patients requesting refills, any comments received by the Internet site, satisfaction as noted by the follow up responses of all patients requesting refills, any comments received by the Internet site survey data collected during the summer of 2003.

Results: Seventy-six percent of Internet requests came from United States, and 24\% from other countries. Most request reviewed by the physicians were granted. All patients requesting medication refills: reported erections sufficient for intercourse and 69\% said their satisfaction exceeded all expectations; none were totally dissatisfied with either the service or medication. Side effect rates were comparable to those in the literature. Comparison of the medical history obtained from Internet patients with that recorded in clinic patients charts revealed that the former was far more complete as were the instructions given to each patient. The dissatisfaction rate of sildenafil seems higher than previously suspected. There were no reported deaths or serious complications.

Conclusions: Internet-based vardenafil and sildenafil prescribing provides the physician with a complete and very detailed medical and sexual history for $100 \%$ of patients without denying any information routinely obtained in a direct patient contact setting. Internet-based practice, which may be expected to require far fewer healthcare resources than traditional settings, rates very high in patient satisfaction. Overall, these data support the safety, convenience, cost and clinical effectiveness of Internet prescribing 
of selected medications. Internet prescribing appears to offer greater and faster patient access and superior patient confidentiality than does traditional office practice. More intensive study of this new style of practice is warranted.

[38]

\section{Low cost telemedicine using cable TV Network \& TV Set}

Mohamed Junaid $^{\mathrm{a}}$, Ravi Chandran ${ }^{\mathrm{b}}$, A. Natarajan ${ }^{\mathrm{a}}$, G. Ravindran $^{\mathrm{c}}, \mathrm{S}$. Maheswaran $^{\mathrm{a}}$ and Nithya Priya ${ }^{\mathrm{c}}$

${ }^{a}$ Kongu Engineering College, India

${ }^{\mathrm{b}}$ Velalar College Of Engineering \& Technology, India

${ }^{\mathrm{c}}$ Annamalai University, Erode, India

Nowadays, the need for specific health support at home is very much required for the benefit of patients and also for the doctors to to follow up after treatment for their patients. There are number of web based communication systems are developed for this application. The access and understanding of web service is very difficult for the society,particularly in rural regions. These systems also ensure high investment cost, technical skills and computers in both ends. The deployment of broadband communication networks is making feasible the provision of home care services with a proper quality of service.

TV and cable TV networks are the mass media, which attract and understand by each and everybody in the society. The proposed method uses an innovative telehomecare technique which runs by using multimedia platform over integrated services consisting of a set top box, and a standard TV set with remote control for patient interaction. Since our government has planned to implement Conditional access TV (CATV) system throughout the country using set top box, this method ensures an innovative, low cost and modern health care system which will be attracted and utilised by the entire society.

This platform allows on-line ECG, BLOOD PRESSURE, TEMPERATURE and HEART SOUND monitoring. Even an expert opinion and interaction between the doctors and exchange of medical images from various speciality hospitals can also be achieved. Usability, and affordability were considered as the important factors in the design and development of its hardware and software components. The home station [HS] or 1st station is composed of two detachable units: the video conference data processing unit and vital signs recording module [VSRM]. The first module is a PC based set top box. The medical center set up is composed of a call center,the patient record database management and peripherals such as printer and scanner. The solution to the better health care delivery to the rural society can be achieved by the above method by installing this scheme in the rural hospitals.

[39]

\section{eHealth Market Players}

\section{Walid Elias Kai \\ Universite Paris Dauphine, France}

Abstract: Due to the economic and social priorities afforded health services in the United States, research on new delivery modalities such as the Internet is gaining in popularity. Claims of the Internet's potential range from a promise to revolutionize the fundamental way health care is delivered to a tool for empowering patients through enhanced interaction with providers (Rice, 2001). Even though a great amount of attention has been given to e-health activity, the preponderance of publications to date has focused on the Internet as a source of health information. However important this form of e-health is, 
this type of service simply does not face the same constraints that must be addressed by those actually delivering health care services or tightly regulated pharmaceutical products. In this paper, we examine e-health by focusing explicitly on the delivery of health care products and services.

Our examination of e-health activity is guided by two broad research questions. First, we ask what the potential is for the development of online health care services by examining its potential in major health care service and product sectors. Second, based upon case studies of two online health service firms, we seek to understand the emerging strategies of firms that are attempting to enter the health care market with an entirely online approach. Our examination of current e-health trends, as well as our two case studies, demonstrates the tremendous potential for health-related commercial activity on the Internet. However, our examination of the barriers facing ehealth from the US health system also pointed out the almost insurmountable challenges. We therefore conclude that a "click and mortar" model may perhaps be the optimal strategy for e-health. The Evolving ePrescribing Market Players: Strategies for Successful Partnerships begins by examining the activities of various healthcare players in the drive to increase physician adoption of handheld devices and ePrescribing applications, evaluating the most likely outcome of these ventures.

The brief examines physician perceptions of pharmaceutical manufacturer-sponsored ePrescribing services, deciphering the feasibility of this strategy. Strategies for Successful Partnerships continues with an analysis of the vendor market, including the viability of various business models and current partnerships and ventures involving pharmaceutical manufacturers. The future decoded section discusses the potential impact of ePrescribing applications on physician behavior and the emergence of data products, both of which are likely to result in ePrescribing and related handheld applications becoming established channels to communicate with physicians. The Partners should be chosen based upon not only the strength of their technology, but on the viability of their business model. Physicians in the US are the most positive about using ePrescribing tools sponsored by pharmaceutical manufacturers.

Conclusion: Physicians are being targeted by strategies initiated by various healthcare players with the intent of increasing the penetration of handheld devices and ePrescribing applications. Such targeting will result in both increased adoption and the creation of a new and powerful channel for impacting prescribing decisions by reaching physicians as they are making these decisions. Additionally, increased ePrescribing market activity will result in the creation of data products with increasing statistical significance over the next three years. The combined result of an improved ability to target physicians at the point-of-care and to collect data on their prescribing decisions will be the emergence of ePrescribing and related handheld technologies as lasting channels to communicate with physicians.

\title{
[40]
}

\section{Empowering Patient Human Process}

\author{
Walid Elias Kai \\ Université Paris Dauphine, France
}

Advances in medical device technologies continue to provide opportunities for better healthcare, and fields such as genomics will offer tremendous potential over the coming years. There has been an acceleration of new knowledge, new drugs, and new opportunities. Information technology continues to undergo radical transformations in the evolution from the room-sized calculators of the 1940s to portable, all-purpose devices supporting communication, calculation, and a wide range of human activities. Capabilities now exist to capture and support various forms of information and communication that were previously either impossible or too inconvenient to be practical. Over the next five years, we 
will see tremendous advances in the form and content of human-computer interaction. New devices and connectivity options will drive the era of pervasive computing, in which people can access information services from the home, office, automobile, and other locations. A focus on human-centric processes is necessary for healthcare organizations to realize the full benefits of information technology advances. The goal of information technology in healthcare is to provide simplified, convenient access to meaningful information for human beings as they communicate and collaborate in the course of providing care. The current rate of technology change, however, often leads to an excess of complex devices and complicated information that provides more cognitive burdens than it relieves. The challenge for information technology in healthcare is to provide processes and techniques to contain the rate of change and variability and thus reduce or eliminate the barriers to effective use of information technology in improving quality and patient safety.

Objectives: A focus on human-centric processes is necessary for healthcare organizations to realize the full benefits of information technology advances. The goal of information technology in healthcare is to provide simplified, convenient access to meaningful information for human beings as they communicate and collaborate in the course of providing care. The current rate of technology change, however, often leads to an excess of complex devices and complicated information that provides more cognitive burdens than it relieves. The challenge for information technology in healthcare is to provide processes and techniques to contain the rate of change and variability and thus reduce or eliminate the barriers to effective use of information technology in improving quality and patient safety.

Study design: Human-Centric Processes Human beings are dynamic and unpredictable, with imperfect memories. We frequently misunderstand symbols, words, and expressions and have insufficient knowledge of new or unfamiliar domains. Advances in technology often lead to excesses of information that tax the human information processing system beyond that which is necessary to accomplish the task at hand. Thus, it is imperative that systems be designed from a perspective that optimizes human strengths and accommodates weaknesses. Three increasingly specific areas that focus on the human element in man-machine systems include human factors, human-computer interaction, and computer-supported collaborative work.

Methods: Nielsens Usability Heuristics Nielsen13 lists a number of design heuristics for the user interface, as shown in Table 8-1. The emphasis on human-computer interaction and usability testing will increase as software supports a greater number of tasks that were previously performed manually. For example, electronic ordering systems should present all the information necessary for a clinician to make the correct decision clearly. Usability testing and evaluation techniques are necessary to measure how well a software system meets these requirements.

Results: Automated hardware and software can benefit the medication use process by helping to reduce errors associated with common causes of medication errors pointed out by Cohen.21 For example, failed communication can be assisted with electronic transmission of orders that have been subjected to validation; drug distribution can be enhanced by using the unit-dose system with bar-coded information, along with proper use of automated dispensing equipment. Typical elements of automation currently in practice include the following: Automated ordering, with validation and screening against drug interactions Physician access to patient and clinical information Bar-coded medication Automated dispensing Automated delivery Automated bedside scanning systems Alerts and other collaborative tools in the event of an error Patient access to information.

Conclusions: Healthcare organizations that focus only on point solutions will introduce additional errors either from automating faulty processes or from failures associated with technology. Leaders of healthcare organizations should focus on system-wide processes and implement technologies appropriately. By focusing on the human element in the system, the full benefits of information technology can 
be realized and errors can be avoided. 1. Implement a system-wide approach to preventing, avoiding, detecting, and recovering from errors. The first step is to understand that complex systems result in errors caused by fragmented processes and organizations. Many of the barriers to error reduction are cultural or psychological. Individuals operating within a punitive environment are often reluctant to admit an error was made. In other cases, the lack of a system-wide approach fails to yield enough information to understand the true cause of an error. Dealing with complexity is something that requires human intervention. In health care, we focus on individual carefulness and expect that to prevent errors, said speaker Martin J. Hatlie, a lawyer and president of Chicago-based Partnership for Patient Safety.9 The bigger and more complex you make it, the more places there are for it to break down. Medical errors are often the result of complex interactions of individuals performing their jobs correctly. The root-cause analysis attempts to address the complexity of a system to uncover the cause, not just the obvious subsequent consequences of the cause. It recognizes that human activities are error prone and includes the human factor in the analysis.22 Implementing such a process may require reorganizing or repurposing current staff and requires collaboration among staff and administration to ensure a nonpunitive culture that learns from errors. At the Luther-Midelfort Hospital, initial investigations revealed approximately 200 to 230 potential medication errors for every 100 charts. 23 Improvement came with an organizational change featuring strong leadership and a focus on individual processes, including admissions, transfers, and discharges.

\title{
[41]
}

A Two-method Evaluation Approach for Web-based Health Information Services: The HealthCyberMap Experience

\author{
Maged N. Kamel Boulos \\ Institute of Health and Medicine, University of Bath, Bath, UK
}

Educational objective: At the conclusion of this presentation, participants should be able to appreciate that different evaluation methods for Web-based medical/ health information services have different strengths and weaknesses and so are not completely effective when used alone, and that only a good multi-method evaluation approach can help put together an adequate, more complete picture of how a service is being used and received by users. (Evaluation methods include questionnaires, semi-structured interviews, video tracking of a sample of users in a usability lab, analysis of Web server transaction logs, and expert panel critique using a standard checklist of usability heuristics. The choice of the specific methodologies used for a particular evaluation exercise depends on the resources (human, financial, time, etc.) available for the evaluation, among other things.)

Study design: A two-method formative evaluation approach was adopted using an online user questionnaire in addition to HCM server log analysis, guided by NIH Web Site Evaluation and Performance Measures Toolkit methodologies among others. The two chosen methods act in a complementary synergistic way, providing a more complete picture about HCM and crosschecking each other (e.g., to verify that the sample of questionnaire respondents is a good representative of the larger set of users logged in server logs).

Methods: HCM evaluation questionnaire covers service usability, usefulness, and user satisfaction issues. It includes a usability task, and a comparative task, comparing HCM to another approach. Both tasks are assessed qualitatively. Thirty-five subjects responded to the questionnaire during the 45 -day period from 18 April-1 June 2002. Sawmill 6.3.8 was selected to analyse HCM server logs covering the same 45-day period of this study. Some service usage details derived from server log analysis, e.g., 
information about top referring search engines/ sites, the geographical provenance of service visitors and the most common browser types/ versions used to access the service, might be more accurate than similar information obtained from a user questionnaire, because of the much larger number of users covered in server logs. Server transaction logs can also provide valuable quantitative information about server traffic and performance, and visitor retention or "stickiness" among other things.

Conclusions: Using more than one method for the evaluation of a Web-based health information service can help putting together an adequate, more complete picture of how the service is being used and received by users (compared to results obtained using only a single evaluation method).

[42]

Quality-assured knowledge on the Internet - does it change professional practice?

Patty Kostkova, Julius R. Weinberg, Gemma Madle and Jane Mani-Saada UK City University, London, $U K$

In recent years, the ever-increasing amount of medical information available online and the increasing availability of the Internet at work as well as at home has significantly changed the way information are used by health care professionals and by general public. However, despite the desire of better informed patients to take an active role in the treatment process, and the need of professionals to keep improving their practice according to new results available in Digital Libraries on the Internet, the reality does not fulfill the expectations. In our research we are seeing answers two questions: Does making the evidence available online result in any difference in the professional practice? Does the accessibility of information make the search for the knowledge any easier? The National electronic Library for Communicable Disease (NeLCD), we are developing at City ehealth Research Centre (CeRC), serves as an excellent research testbed for investigating these issues.

NeLCD is one of the Specialist Libraries of the NeLH, is providing an on-line, evidence-based, quality-tagged Internet portal to the best available evidence on prevention, treatment and investigation of communicable disease. In addition to providing a single point to available electronic resources on communicable diseases, the key value of the library is in a quality appraisal of all posted documents. This procedure is conducted in collaboration with all major professional societies and expert committees in the area of communicable disease. Each document considered the best available evidence on a particular subject is assigned a quality tag, by a member of a professional society. The quality tag consists of a bottom line critically summarizing the paper, and a checklist, which answers brief questions about the methodological issues, level of evidence, potential biases and applicability of the results. The resultant quality tag and a signature of the particular society are attached to the document and made available through the NeLCD.

In addition, the site will provide an on-line forum to allow professionals to comment on the qualitytagged papers, as well as to discuss important issues on-line as soon as they arise. Furthermore, the site will provide professional commentary on information hitting the news, instant dissemination of outbreak control procedures, moderated discussion list, regular newsletter and user alerts on requested issues.

As for data representation, The NeLH is to store evidence-based medical information on clinical and non-clinical subjects, medical teaching material, lectures and slides.

However, there is currently no common agreement on ontology, nor agreed standards in health care (coding standards, data representation standards and common legal and ethical recommendations). For example, there is no common internationally accepted clinical coding scheme - currently, several coding systems are being used by different organizations: MESH, CTLV3/SNOMED and ICD10. This is not 
only a UK but an international issue. The NeLCD team is involved in the EU Agentcities initiative (www.agentcities.net), in particular the Health care working group looking at, among others, existing ontologies in participating EU countries and finding options for common agreements. However, MESH (http://www.nlm.nih.gov/mesh/2002/index.html) - Medical Subject Headings was chosen by the NeLH team as the underlying medical ontology for indexing documents in the library. As it is the most commonly used among medical librarians in the UK and is the standard used for indexing in Medline. In the Dublin Core (DC) definition, keywords appear as "Subject". Agent support for user customisation and personalization of the indexing scheme was discussed in. In order to support a user-customizable search, documents in the library need to be precisely described. The Dublin Core Metadata initiative (http://www.purl.org/DC) defines a list of fields characterizing an electronic document for cataloguing and search purposes. The NeLH adopted and extended this framework to better meet the requirements of quality and "up-to-date-ness". Every document in NeLH will be described by this adapted DC, called an "electronic catalogue card".

One of the key applications of Intelligent Agents, a fast-growing area in software technology, is information retrieval and assistance in searching information in the Internet. In addition, agents autonomous behaviour enables them to assist in various search-related processes on the users behalf, however, without human intervention. These features make them particularly exciting for health care applications, and in various health-related fields in general. However, currently, there is no common agreement on ontology, nor agreed standards in health care (coding standards, data representation standards and common legal and ethical recommendations). Agents in NeLCD are performing a number of tasks - tracking the documents in the library during the appraisal process, search and distributed cross-search, and customisation and profiling.

In this talk, our experience with NeLCD development will be presented, technical issues briefly described and the uptake among UK communicable disease professionals and the obstacles we discovered during the process will be outlined.

\section{[43]}

\section{Review of Different Aspects of Bioterrorism for a comprehensive medical informatics-telemedicine system}

Anand Kumar
University of Pavia, Pavia, Italy

This paper presents an abridged version of a status report being prepared by the author in order to be submitted to the key academic institutions and hospitals in the state of Pennsylvania, spearheaded by University of Pennsylvania, for creating the blueprints of a project proposal to the newly established Department of Homeland Security in the United States. The project would simulate different sorts of bioterrorist attacks in the Greater Pennsylvania area and would entail the study of different aspects of usage of medical informatics and telemedicine including emergency data delivery systems, electronic communications between the healthcare practitioners in a critical situation, psychocognitive aspects of bioterorrism and of telemedicine, vaccine information systems integrated with drug databases, population health technology and public health law related aspects, role of clinical genomics and bioinformatics etc. The author has been involved in the review of more than three thousand articles and abstracts, multiple newpaper articles and two hundred websites which directly or indirectly deal with bioterorrism. Due to a lack of space, some of most recent and important articles are mentioned here, with a brief mention of 
certain key aspects which should form the core of a comprehensive medical informatics-telemedicine system for the management of bioterorrism.

\title{
[44]
}

\section{Implementation strategies for telematic project}

\author{
Marc Kurepkat ${ }^{\mathrm{a}}$, Bertram Haussler ${ }^{\mathrm{b}}$ and Cornelius Barthels Barthels ${ }^{\mathrm{a}}$ \\ ${ }^{a}$ Clinische Studien Gesellschaft, Berlin, Germany \\ ${ }^{\mathrm{b}}$ Institut fur Gesundheits- und Sozialforschung, Berlin, Germany
}

The path of new telematic applications to maturity and to market is often via integration in a project. The successful management of telematic projects is therefore especially important for the development of this sector of the health industry. As the benefits of telematics become more obvious to all players in the health system, any remaining scepticism will focus increasingly on practical implementation and performance. Every instance of non-implementation, project failure and technical error will fuel critics claims that the potential of telematics for procedural improvements in the health system is only theoretical and does not hold up in practice. There have been cases of initially enthusiastic medical practitioner users who have lost faith in technology in the course of a telematic project.

This is doubly regrettable since not only is a project participant lost to the cause but also a potential future user of the tried and proved application that the project aims to produce. Shortcomings in the infrastructure of a medical practice represent a significant obstacle to the success of telematic projects. In implementing fully developed telematic applications participants will often come up against a combination of three typical user problems: inadequate hardware; unsuitable software; lack of knowledge. To overcome these problems telematic projects and products usually focus on providing suitable software components. As a rule only minimum hardware requirements for telematic products are defined and the relevant hardware is not made available, while attempts to remedy the knowledge shortfall are often unsatisfactory. The reason for this lies not so much in the applications own teething problems as in the often shaky office models used for telematic products. Participants will be reluctant to invest as a result. Solving problems that arise while the project is running is rarely feasible, since it involves additional investment in new hardware, training etc.

The result is procedural deterioration or project failure. A uniform solution is needed for this common problem. One way of improving chances of success for telematic projects is through a rigorous and systematic selection of users by means of standardized infrastructural user assesssment. Infrastructural assessment is a procedure for gathering information using SOPs and assessment tools in the form of a questionnaire and criteria for user classification. On the basis of information so gathered potential users are divided into three groups: Group A: successful participation is (highly) likely without any further enhancement of available infrastructure; Group B: successful participation is (highly) likely with considerable enhancement of available infrastructure; Group C: successful participation is not likely, even with considerable enhancement of available infrastructure. Users in Group C should be excluded from the project. Exceptions could be made where a Group $\mathrm{C}$ candidate elects to carry the cost of a very attractive project with longterm user benefits. Users in Group B should only be included in the project when there are insufficient Group A participants and then of course only as the budget permits. The costs for the assessment must be taken into account in project budgeting. The Clinische Studien Gesellschaft and the IGES-Institut have already developed such a system for infrastructural assessment of medical practices. It has been used for three projects and fine-tuned on the basis of the experience so gained. 


\title{
[45]
}

\section{Deployment and clinical evaluation of a Web-based self-help guide for the treatment of Bulimia Nervosa}

\author{
Tony Lam ${ }^{\mathrm{a}}$, Isabelle Carrard ${ }^{\mathrm{b}}$, Patrick Rouget ${ }^{\mathrm{b}}$, Thierry Raguin ${ }^{\mathrm{a}}$ and Mickael Cappozzo ${ }^{\mathrm{a}}$ \\ ${ }^{a}$ NetUnion SARL, Switzerland \\ ${ }^{\mathrm{b}}$ Hopitaux Universitaires de Geneve, Switzerland
}

Abstract: Eating disorders are a common source of psychiatric morbidity among young women [1]. In order to respond to such problems, it is urgent to develop and evaluate more accessible treatment methods. Self-help manuals are one solution. Research on self-help manuals for the treatment of Bulimia Nervosa (BN) has already been conducted in Anglo-Saxon countries, where effectiveness of such methods is recognised [2-7]. These manuals are based on cognitive and behavioural therapy (CBT) and provide step-by-step methods to face eating disorders by progressively recovering self-control over one's food behaviour. Self-help manuals offer an innovative solution for meeting the increasing demand for specialised treatment. However, patients working alone with these manuals need to be strongly motivated to succeed. The added work of recording, and organising daily records could also be an obstacle to success.

Using online versions of these self-help manuals could bring new benefits [8]. Online systems allow patients to submit exercise directly thus limiting the amount of paperwork for both the patient and the health care provider. Therapists can monitor and track their patient's progress easily, and the system could provide more flexible channels of contact with the patient. Web-based applications can also reach people that otherwise would not seek help, due to practical or constraints such as physical distances, psychological constraints, or a sense of shame (BN is most of the time linked with shame) [9].

The goal of this study is to increase research evidence of the advantage of using online self-help manuals [8]. Our main hypothesis is that the online delivery of self-help manuals can be effective for the treatment of BN. The study also seeks to determine clinical predictors for treatment outcome from the collected data.

The study is part of Salut! (IST-2000-25026), a 39-month project funded by the European Commission under the 5th Framework Programme and by the Swiss Federal Office for Education and Science (OFES). This project, started in January 2001, aims at developing Web-based and mobile tools for diagnosis, treatment, and prevention of eating disorders. The online Self-Help Guide (SHG) for outpatient treatment of $\mathrm{BN}$ is one of the main results of this project. Clinical trial activities for the evaluation of the SHG started in October 2002 and will continue until January 2004. Trials are conducted in Switzerland, France, Spain, and Sweden in French, Spanish, and Swedish. The multi-lingual platform of the SHG enabled the project to rapidly deploy multiple versions of the SHG, facilitating the extension of research and trial activities to other countries.

Conclusion: First results of the clinical trials are promising and the online application has exhibited several advantages. Patient acceptance is high. In addition, the multi-lingual scope of the application gives us the opportunity to deploy the SHG in most European countries and to easily expand its use to other languages. The opportunity for providing this service to a minority population is also perceived to be an unexpected benefit (e.g. providing the Spanish version of the SHG to the Spanish-speaking communities in Sweden).

Moreover, the Web-based SHG is based on a generic platform for the delivery of e-health / e-mental health services. This platform, by its modularity, could allow the rapid deployment of other self-help applications based on CBT, e.g. panic disorder, pathological gambling, etc. 
Early results also indicate that making some mobile SHG modules could also extend accessibility and convenience. A prototype for accessing some of the SHG tools via WAP has been implemented, and we are starting to look at ways to integrate mobile technologies more completely in the platform to provide seamless and secure access to the SHG.

\section{References}

[1] W.S. Agras, The consequences and costs of the eating disorders, The Psychiatric Clinics of North America 24(2) (2001), 371-379.

[2] J.C. Carter and C.G. Fairburn, Cognitive-behavioral self-help for binge eating disorder: a controlled effectiveness study, Journal of Consulting and Clinical Psychology 66(4) (1998), 616-623.

[3] P.J. Cooper, S. Coker and C. Fleming, Self-help for bulimia nervosa: a preliminary report, International Journal of Eating Disorders 16(4) (1994), 401-404.

[4] P.J. Cooper, S. Coker and C. Fleming, An evaluation of the efficacy of supervised cognitive behavioral self-help bulimia nervosa, Journal of Psychosomatic Research 40(3) (1996), 281-287.

[5] J. Treasure, U. Schmidt, N. Troop, J. Tiller, G. Todd, M. Keilen and E. Dodge, First step in managing bulimia nervosa: controlled trial of therapeutic manual, British Medical Journal 308 (1994), 686-689.

[6] J. Treasure, U. Schmidt, N. Troop, J. Tiller, G. Todd and S. Turnbull, Sequential treatment for bulimia nervosa incorporating a self-care manual, British Journal of Psychiatry 168 (1996), 94-98.

[7] C. Thiels, U. Schmidt, J. Treasure and R. Garthe, Guided self-change for bulimia nervosa incorporating use of a self-care manual, American Journal of Psychiatry 155(7) (1998), 947-953.

[8] C. Williams, New Technologies in Self-help: Another Effective Way to Get Better? European Eating Disorders Review 11 (2003), 170-182.

[9] U. Schmidt, Getting Technical, European Eating Disorders Review 11 (2003), 147-154.

[10] Garner, EDI-2: Eating Disorders Inventory - 2, 1991.

[11] Derogatis, SCL-90R: Symptom Checklist - 90 revised, 1977.

[12] American Psychiatric Association, Diagnostic and Statistical Manuel of Mental Disorders, Fourth Edition, 1994.

[46]

Can a one-person-operated, non-commercial website have any impact on world-wide health care?

Al Lodwick ${ }^{\mathrm{a}}$ and Diane E. Hindman ${ }^{\mathrm{b}}$

${ }^{\mathrm{a}}$ Lodwick Publishing, Pueblo, USA

${ }^{\mathrm{b}}$ Canada Ontario Ministry of Health \& Long-Term, Canada

Objective: To determine if a one-person operated, non-commercial website (www.warfarinfo.com) is able to have an impact on world-wide health care.

Study Design: Determine the number of visitors to the website by country and the number of pageviews by those visitors. Compare this data to the population and healthcare spending for those countries.

Methods: The visitor, number of countries with visitors and pageview data were drawn from the control panel of the hosting internet service provider (ISP). Population data was obtained from www.cia.gov. All of the countries were analyzed to determine number of visitors per 1 million population. Then the top fifteen countries in this category were then classified as whether they were large industrial small island or isolated nations. Healthcare spending data was found at www.oecd.org/dataoecd/1/33/2957315.xls. The thirty largest health care spending countries were compared to the fifteen largest per capita visitor countries. We analyzed the countries having the largest number of pageviews per visitor as to the type of country (large industrial, small-island, isolated). Then we compared the ten countries with the largest number of pageviews per visitor with the thirty countries with the largest per capita health care spending. 
Conclusions: The countries with the largest numbers of visitors per capita are primarily small islands or isolated countries that were primarily not large per capita spending nations in terms of healthcare. The countries with the largest numbers of pageviews per visitor were exclusively small island or isolated countries that were not among the largest spending per capita nations on healthcare. A one-person operated, non-commercial website (www.warfarinfo.com) is able to have considerable impact on the healthcare of people living in small island and isolated nations that do not have large per capita expenditures on healthcare.

\section{[47]}

VIPS, a Web-based simulator of medical consultations: An electronic counterpart of Standardized Patients?

Vanya Loroch ${ }^{\mathrm{a}}$, Mathieu Nendaz ${ }^{\mathrm{b}}$, Robert L. Thivierge $^{\mathrm{c}}$ and Marc-Andre Raetzo ${ }^{\mathrm{d}}$

${ }^{a}$ University of Geneva, Department of Internal Medicine, Switzerland

${ }^{\mathrm{b}}$ Unit of Research and Development in Medical Education, Switzerland VIPS

${ }^{\mathrm{c}}$ University of Montreal, Canada

'Switzerland Groupe Medical d'Onex

Standardized Patients (SPs) are individuals trained to simulate an actual patient in an accurate and reproducible manner. Encounters with SPs are used extensively to train and assess clinical and communication skills of medical students, residents, and physicians. While the benefits of SPs are well documented, the overall quality of this educational methodology strongly depends on actor training and availability, as well as on the reproducibility of the information provided to the physician. In addition, the relative high cost of SP development and logistical constraints may constitute a barrier for large scale implementation, in particular within the framework of Continuing Medical Education (CME).

VIPS (Virtual Internet Patient Simulation) is a Web-based simulator of medical consultations that may complement and/or substitute SPs during formal medical training and as a CME offering. A VIPS consultation is similar to an SP encounter in that the learner is faced with a succinct chief complaint and must work up the virtual patient in an open-ended manner and in the absence of any a priori information which may influence his or her actions. At the end of the consultation, the learner's performance is evaluated step by step, and each item (question or decision) is scored according to the evidence found in the literature and directly accessible through Web deep-linking.

Unlike SPs, VIPS does not assess interpersonal/communication skills but rather focuses on clinical reasoning by linking peer-reviewed publications to every question asked and every decision made by the learner during the virtual consultation. As a consequence and because it relies on a controlled database of medical knowledge, VIPS represents a highly standardized tool particularly aimed at evaluating the data collection and decision-making processes.

The new software implementation of the VIPS system will be presented. The similarities and differences between VIPS and SPs will be discussed in the light of the studies being conducted at the University of Geneva and the University of Montreal Medical Faculties. Because the VIPS system is flexible and may apply to physician training and assessment at different levels of medical experience and at low costs, opportunities for international collaborations will also be presented.

\section{[48]}

Sharing clinical documents in a national care provider network to support community based medicine 
Christian Lovis, Stéphane Spahni and Antoine Geissbühler Service of Medical Informatics, University Hospitals of Geneva, Switzerland

Abstract: One of the key factors for improving the quality of care in a community-based healthcare network is the preservation of the continuity of care. Continuity of care implies the ability of taking care of patients in a coherent and pertinent manner. It is very dependant of the integrity of the information flow, especially when several care providers are involved. Promoting information flow in the very sensitive field of patient data however can be in contradiction with ethical and legal environment. We describe the implementation of a secure server, using a Swiss protected network over Internet devoted to care providers and allowing the sharing of clinical documents in a very tight patient-care provider relationship. The patient can choose which documents will be made available to the care provider he selects, thus empowering his control over its own medical data. This is the first step to establish a distributed, patient-controlled, virtual patient record (VPR). Virtual patient records provide a means for integrated access to patient information that may be scattered around different healthcare settings.

[49]

\section{Development of a methodology to evaluate the impact of a medical digital library on user knowledge, attitude and behaviour}

Gemma Madle, Patty Kostkova, Jane Mani-Saada and Julius Weinberg

City University of London, London, $U K$

The objective of this paper is to propose a methodology for evaluating the impact of medical digital libraries on user knowledge, attitude and behaviour.

Recent years have seen an explosion in the amount of health information available to patients on the Internet, but is all this newly available information making any difference to patients' behaviour? Previous usability testing of digital libraries and websites has focused almost exclusively on the graphical interface issues and organization of information within the DL or website, rather than their role in changing work practices or behaviour.

Medical digital libraries are essentially life critical applications enabling professionals to stay up to date. They have the potential to change working culture creating new types of professional relationships and communities based across distances, hierarchy and other traditional barriers. Some recent research indicated that some senior clinicians are reluctant to provide access to evidence-based information to junior staff, one even suggesting that they should learn on the job instead! [1] The general public now have easy access to medical information and research evidence that they may present to their clinician. They are able to participate more fully in the management of their health, sharing in decision-making [2]. The balance of power is shifting but it requires skill on the clinician's part to adapt to patient preferences. They have to know when to hand over decision-making, when to share it and when to take a paternalistic approach [3]. Therefore it is important that $\mathrm{w}$ an measure the effectiveness of medical DLs and health information websites in changing the knowledge and attitudes of both health professionals and the public so that the healthcare system can exploit these resources to its best advantage.

We propose a methodology combining transaction log evaluation and pre and post-use questionnaires for evaluating the impact of the National electronic Library for Communicable Disease on users knowledge, attitude and behaviour. Transaction logs provide information about the path a user takes through a website. We can find general patterns in use e.g. most commonly visited pages, search terms used, time spent on a page etc. We can also employ a technique known as microanalysis, analyzing use of the 
library by a small number of individual users. This provides a clearer picture of individual user behaviour when in the library, rather than general trends [4]. Questionnaires before and after using the library allow us to evaluate user knowledge, attitude and intended behaviour as well as usability of the library [5]. Comparing how users navigated the library, where they went and what documents they requested with how their knowledge and attitude changed, as well as the usability of the library, enables us to build a picture of the impact of the library on users and potentially the impact on their work.

We are currently analyzing transaction $\operatorname{logs}$ of the pilot NeLCD website. We have also completed a study investigating the impact of a subsection of the NeLCD on public knowledge and attitudes [5]. This study provided evidence that medical digital libraries can change knowledge and attitudes. The combined methodology proposed will be used to evaluate the impact of the new NeLCD website, due to be launched this Autumn.

In conclusion, this paper has presented a methodology that will be used to evaluate the impact of a medical digital library on user knowledge, attitude and behaviour. Such evaluation is essential to ensure the library is meeting user needs and helping to improve the quality of care received by patients.

\title{
References
}

[1] A. Adams and A. Blandford, Acceptability of Medical Digital Libraries, Health Informatics Journal 8(2) (2002), 58-66.

[2] Health on the Net Foundation Evolution of Internet Use for health purposes, http://www.hon.ch/Survey/FebMar2001/ survey.html, March 2002.

[3] G. Elwyn, A. Edwards and P. Kinnersley, Shared decision-making in primary care: the neglected second half of the consultation, British Journal of General Practice 49 (1999), 477-482.

[4] D. Nicholas, P. Huntington and P. Williams, Micro-mining log files: a method for enriching the data yield from Internet $\log$ files, Journal of Information Science (in press: Summer 2003).

[5] G. Madle, P. Kostkova, J. Mani-Saada and J.R. Weinberg, Evaluating the changes in knowledge and attitudes of digital library users. To be published in the proceedings of the 7th European conference on Research and advanced technology for digital libraries - ECDL 2003, Lecture Notes in Computer Science, Springer Verlag, 2003.

\section{[50]}

\section{e-Health \& Learning: The Bangladesh experience}

\author{
Elena Murelli ${ }^{\mathrm{a}}$ and Theodoros N. Arvanitis ${ }^{\mathrm{b}}$ \\ ${ }^{\text {a } C R A T O S ~ C a t h o l i c ~ U n i v e r s i t y ~ o f ~ P i a c e n z a, ~ P i a c e n z a, ~ I t a l y ~}$ \\ ${ }^{\mathrm{b}}$ The University of Birmingham, UK
}

We live in a world of inequality and diversity. This world is divided roughly into three kinds of nations: those that spend lots of money to keep their weight down; those whose people eat to live; and those that dont know where the next meal is coming from. At the beginning of the new millennium this is the reality where we live, but there are new technologies, which can improve our life. In the twenty-first century there will exist a predominately information and knowledge-based society, where every country hopes to achieve its goal of social and economic development, including full education, proper food security, decent health, genuine gender equity and respect for cultural pluralism. Today, the most important problem is to attain and sustain these goals, therefore every continent should concentrate in building its own Information Society, until a global village is reached. Developing an adequate and affordable Telecommunication infrastructure can help to close the gap between the haves and the have-nots in health care - Dr Hiroshi Nakajima, Director-General of the World Health Organization (WHO). 
The use of information and communication technologies can become a common tool for all health care professionals and patients. Health telematics applications could be as natural as the stethoscope and the telephone, nowadays. Telemedicine is no longer just a playground for dreamers, enthusiasts and pilot programs. It has become a strategic tool for those countries where, failing economic trends, decades of political instability and low levels of development, the health sector lags far behind its counterparts in many other countries of the world, especially within the more industrialized nations. In a utopian world, every citizen has immediate access to the appropriate specialist for medical consultation. In the real world this cannot even be a dream. It is a fact of life that All men are equal, but some are more equal than others. We are at present, unable to provide even total primary medical care in the rural areas. Secondary and tertiary medical care is not uniformly available even in suburban and urban areas. Incentives to entice specialists to practice in suburban areas have failed. Healthcare worldwide has to respond to new and growing needs originating in demographic and socio-cultural changes, progress based on medical research and technology and, last but not least, globalisation. Within these constraints e-Health has a great potential becoming a key factor for a widespread healthcare provision. Technologies like networked electronic health records, broadband communication and secure networks enable the delivery of "ubiquitously available" and enhanced healthcare services to patients.

The massive deployment of information technologies changes the access to and the nature of healthcare at rapid pace. The potentials of e-Health for a support of a patient-focused and future-oriented healthcare are nowadays universally recognised. Telecommunication and IT-applications facilitate communication and information, simplify diagnostic and therapeutic processes and enable better care to be provided in the patient's private environment. Many promising approaches resulting in the optimisation of the treatment of patients by establishing integrated and comprehensive healthcare networks are in existence. Hence, Telemedicine, Health Telematics and e-Health attract increasing attention of the health policy makers, social security funds and healthcare providers throughout the world. It is the ultimate goal; optimise the access to and the quality of the healthcare while simultaneously reducing or at least limiting the costs. In Bangladesh, the status of healthcare is similar to the one of many developing countries: medical practitioners in Bangladesh often operate in relative isolation, dealing with diverse health care needs, many of which arise infrequently because of the small populations served. Worldwide there is difficulty in retaining specialists in non-urban areas. The distribution of specialists in Bangladesh is indeed lopsided. There are more neurologists and neurosurgeons in some cities; similarly tertiary care hospitals are also concentrated in pockets with large segments of the population having no access. The increasing availability of excellent telecommunications, infrastructure and video conferencing equipment will help provide a physician where there was none before.

The rapidity and the ease of access to medical information are essential conditions for the quality of the help to the patients, for the medical researches and for the checking of the developments in the health service. But in many areas of the developing countries it is really hard, if not impossible, to obtain some information because of the poor telecommunication infrastructures, of the economic conditions and of the natural disasters. Beyond the particular needs of the different country-specific healthcare systems there seems to be a common understanding of the priority for integrated, cross-sectorized and patient-oriented healthcare activities. This integration, in the best manner, meets the needs of health benefits taking into account quality of care and economic feasibility. The necessary information, communication and security infrastructure plays a key role in meeting these needs. The e-Health \& Learning Project would like to actively promote the idea of a widespread deployment of e-Health into daily medical practice with the use of e-learning tool to train health operators and keep them informed about new disease and techniques. 
The project has the aim to promote international co-operations to fulfil this mission. The creation of a Medical Portal and a series of seminars is seen as an eminent step forward to improve international co-operation and understanding, being a basis for the worldwide implementation of secure medical networks, cross-sectoral communication and the provision of high-quality websites for the health-related information of consumers and patients. The focuses of the e-Health \& Learning project are to provide knowledge and instruments useful to the development and the management of health information systems, to the automatic support to clinical research, epidemiological surveys, teleconsulting and telediagnosis services and integration between health infrastructures in Europe and Asia, in order to give to the patients a better and faster medical treatment and care. Students, researchers, ITC professionals and health operators from public and private institutions (national and international), in particular from Europe and Asia will attend a series of seminars delivered via videoconference using a VSAT and the Mymensingh regional node.

These seminars will be especially addressed in areas, which are among the most central issues in information and communication technology for health care today. Through videoconferencing or videostreaming plus chat sessions, doctors at remote clinics can communicate with medical centres, consult with each other, and effectively exchange test results, high-resolution images, and other vital information. Following the seminars doctors from different locations can interact directly with each other in live, twoway videoconferencing sessions, while simultaneously exchanging data files and browsing the Mobile Portal. The participants will have access to a Medical Portal, the first of its kind to facilitate the medical community in Asia and Europe in dealing with the healthcare issues in Bangladesh. The Agreed Strategy is on the Evidence-based approach in Medicine that allows professionals to learn and make informed decisions, based on current clinical and healthcare evidence (epidemiological related research, clinical trials, statistical information, published papers and other literature, etc.).

The proposed seminars should go away from the textbook metaphor and encourage the participants to enhance the learning with evidence-based material. There are three modes for the delivery of the material: 1. The live seminars with Q\&A session. 2. Pre-recorded seminars with additional web based material. MS Producer will allow the development teams to create the material and follow an Evidence-based approach. 3. Standalone web-based learning (access through the portal) All modes share a common interest in diagnosis, prevention, and therapy cycle as well as in telemedicine; in addition, in all three modes of learning there will be a Question and Answer (Q\&A) session and a form of assessment/evaluation by the participants. Finally, all participants would need to access resources and material on the Net.

The e-Health \& Learning Project, through its Medical Portal and videoconference seminars, is bringing the relatively new concept of Telemedicine in Bangladesh and is also modifying them according to current status and needs. Such technology is appropriate to support the development of similar services through the local national healthcare service, while it is envisioned to develop extensive network of community welfare centres, which will aid in medical treatment and also help in other issues like education, agriculture, economic development and public health in all developing countries in the area and beyond. In addition to the above benefits, the expertise in e-Health can be transferred through in e-learning and e-commerce to improve the overall wealth generation and standard of living in developing countries. In this cyberspace context, the developing countries, thanks to a mixture of adoption of inventions and of the national cultural values, as well as the co-operation between all the nations, will be able to have access to information and knowledge which can be used for the development of the country and eliminate the gap between the rich and the poor. The Net helps with the globalisation process, nevertheless it escapes all kinds of control, neither public authority nor the most important businessmen can control the flow 
of information transmitted on the Internet. The approach to follow is a convergence between the world of telecommunication infrastructures and legislation in order to define a platform used by everybody to have access to the Internet in a flexible and transparent way, without violating the Human Rights. In conclusion as Muhammad Yunus said, we think that in a human and civil society there is no place for poverty and I hope that the use of the Internet will help to make this a reality soon.

\title{
[51]
}

\section{The Comprehensive Community Health Information System: An Experiment in Regional Inter- sectoral Program Planning and Priority Setting}

\author{
Cordell Oren Neudorf \\ Saskatoon Health Region, Saskatoon, Canada
}

In public health it has long been realized that our health is determined by a broad set of factors including our education, social supports, early childhood experiences, environmental factors, employment, and income. The health system on its own probably is responsible for about $20 \%$ of our overall health or well-being. This complex interplay of factors does not only affect the individual, but often has its influence at the family, group, community or societal level. In order to maximize the potential health status of the individuals within a community, the various partners providing the major human services need to see their work as part of a larger common good, and work together with the community members to better understand the health and social priorities of their community. Once common priorities are defined by these service sectors and validated by the community members, potential joint solutions can be sought. In Saskatoon, a group of Human Service providers have come together to do joint information sharing and find common goals and priorities.

This Regional Intersectoral Committee (RIC) includes Health, Social Services, Justice, Education, and representatives from municipal, provincial and federal governments. As this group shared information, it became apparent that often the individual areas had similar information needs, but did not have the resources to secure the information, or analyze it. Often the required information was out of date by the time it was shared by one member, and it had different geographic boundaries, or different age groupings, than that required by the other members. To solve this problem, the Comprehensive Community (Health) Information System was proposed by the Health Sector. In prototype, this system is designed to take low level aggregate, de-identified information shared by each of the sectors, and post it on a secure internet site as a shared resource. The interface is designed to be very flexible to allow for different levels of access or different ways to view the output according to: -differences in the user (community member, researcher, program manager, executive) -differences in geography (each sector can view others data by their own geographic boundaries within a region, or by other defined boundaries such as neighborhood, or rural municipality) -desired level of detail (aggregation or disaggregation of factors such as age, sex, geography, ethnicity) -frame of reference (different aggregations of indicators can be chosen, depending on what makes sense for the user, including a Healthy Communities framework, a Health Determinents Framework, and a Quality of Life Framework).

Depending on the users needs, and the data limitations, data can be viewed by table, map, or graph, or in customized pre-analysed views such as a neighborhood profile. The contributing partners have each helped shape the prototype design, and are now involved in producing a fully functional system by contributing more data and suggesting more interfaces. The final design will allow for real-time querying of the partners shared databases via a secure portal, with different levels of access depending on need and various privacy and legal constraints. Current work includes defining the metadata behind each others 
data, agreeing on data definitions, defining the various formats used by partners, agreeing on data sharing rules and limitations and building trust and understanding between analysts and administrators in each of the sectors. Even in prototype stage, this tool is being used by the partners to help define priorities for joint policy work and program planning and evaluation in the city core.

The Health Sector has also used the system for its health Status reporting, and for planning the location and content of new primary care centers in the region. The system is designed for growth, as more data is contributed by partners, more partners join, and researchers contribute results of their analysis of the data, or their community research. It is envisioned that it will expand to include a qualitative research data repository as well, searchable by annotated bibliography. By sharing data with each other and then liberating that data in various forms according to the type of user, each agency can get access to more information, in a more meaningful, useable form, and concentrate their limited resources on specific analyses, and on intersectoral research with shared objectives. The community members also can access much of the data to use in their own advocacy. A working prototype is available for demonstration, and a conceptual model and is being developed for more generalized use. Various design details are mapped out and available for use.

\section{[52]}

\section{Indian MEDLARS Centre: Internet and biomedical information for the Indian Medical Profes- sional}

\section{Naina Pandita, Shefali Sushil Dash}

India National Informatics Centre, New Delhi, India

The Indian MEDLARS Centre (IMC) was set up in 1986 with the objective to meet the ever-increasing information needs of the medical professionals in the country. These professionals, who had to depend for information from their institutional/college libraries, had to look up voluminous printed resources which could be a tedious and time consuming task. IMC opened up vistas of information access for these professionals by providing information from National Library of Medicines (NLM) MEDLARS databases. With the Internet making in-roads, information became freely available at the click of the mouse. At this point IMC shifted its focus from content providers to content generators and developed services, accessible over the Internet, for the Indian medical professionals. A bibliographic database of 75 peer reviewed Indian biomedical periodicals was developed, which included journals not covered by any other international database.

This database, IndMED, is accessible free of cost from IMCs website, http://indmed.nic.in to the professionals within and outside the country. Simple and advanced search modes enable the professional to have a comprehensive retrieval. With indexing of journals being done using Medical Subject Headings (MeSH) this database has been designed on the same format as NLMs MEDLINE database. IMC has also developed a Union Catalogue of Indian Biomedical Periodicals, which provides the holdings data of 180 biomedical libraries in the country and this is accessible free of cost from http://uncat.nic.in. This database can be searched by journal names or library names and provides details of the availability of the journals in these libraries.

The Centre is in the process of developing an Online full-text database of Indian biomedical periodicals and this would cover the 75 journals indexed in IndMED. This database, medIND would also be accessible free of any charge to the medical professionals and would serve as a one point access to all peer reviewed Indian biomedical literature. The Centres award winning website, ranked amongst the top 5 Indian health 
sites by Google directory, in addition to providing access to Indian biomedical literature, also serves as a portal to biomedical websites and e-journals.

This includes links to NLM databases and other sites, which are of relevance not only to the professional but also to the consumer, medical library professional and nursing professional. IMC conducts training programmes in information retrieval techniques enhancing the searching skills of medical professionals and making them more proficient in searching the biomedical resources available over the NET; the training material can be downloaded from IMCs website with a CD version also being made available. In this manner the Indian MEDLARS Centre has been and is serving the needs of the medical professionals as well other health care professionals using the Internet as the medium for information dissemination.

[53]

Internet health support groups: Their uses, formats, benefits, risks, falicitation, and research
approaches Elizabeth Ann Pector

Spectrum Family Medicine, Naperville, USA

Objectives: This review was conducted to determine the extent of participation in Internet support groups, to describe support group structure, to document benefits and risks of participation in online groups, to identify important considerations for moderators and researchers in online support groups, and to find resources for both users and moderators.

Study design: A comprehensive review was undertaken via literature and web search. Methods: Literature search was conducted on Medline for publication dates 1996 to 2003, and on Psycinfo for publication dates between 1992 and 2002, using the keywords 'support, self-help, Internet, online, group, efficacy, outcome'. A supplemental Internet search using Google was performed to identify sites that addressed online support groups.

Results: Computer-mediated-communication (CMC) self-help groups are used by 9 to $46 \%$ of patients who access the Internet in search of health information. Anyone with a computer and Internet access can find continuous support in one of thousands of groups. Internet support is valuable for patients with rare, disabling, or stigmatizing disorders, and for caregivers whose responsibilities prevent meeting attendance. Debilitating yet poorly understood disorders such as multiple sclerosis or chronic fatigue are popular topics for online support. Men and women, ethnic minorities, senior citizens, and low-income users have all received benefit. Men may use CMC support more than FTF support. Technological and disability barriers can be overcome. Computer-mediated-communication (CMC) groups exist in many formats. A group's software structure influences the style of participants' interaction, group cohesiveness, and the degree of empathy expressed. Synchronous group formats include Multi-User Domains, chat, email focus groups, web telephony and Internet videoconferencing. Asynchronous group formats range from patient-to-patient email to comprehensive multimodality patient education and communication sites. Stand-alone message boards, newsgroups, and listservs are popular.

Hospitals may provide a significant benefit to frequently admitted patients and their caregivers via access to online support. Several authors have described features that enhance functionality and utility of support networks. Group structure needs to be compatible with computer hardware and software available to most users, and must take into account users? linguistic and computer literacy and limitations related to their health, age or other factors. Introductory training in keyboarding, computer use and research skills may help some populations. When interactive support is part of a comprehensive system, other components such as health information databases, FAQs, restricted-access biographical statements 
about members, and 'ask-the-expert' modules can be included. These provide important background to newcomers while preventing repeated group discussions of basic material. Separate areas within a website can be considered for crisis and for day-to-day messages. Message analysis reveals consistent themes of support, information exchange, sharing of experiences, advice, empathy, catharsis, and advocacy. Empathy and a sense of community are successfully achieved online. Tone varies from factual to emotional. Several aspects of CMC groups are unique when compared to FTF groups.

Anonymity filters nonverbal cues, including personal characteristics that might disturb others. It also facilitates discussion of sensitive topics and leads to rapid disclosure of intimate details. Writing has long-term health benefits for chronic illnesses, and group participants feel they benefit from reflecting on word choice as they write their stories. Lurking occurs in 75-95\% of some groups. Those who do not post regularly still feel they learn valuable information. Some may lurk to familiarize themselves with group culture before writing. Asynchronous discussions frustrate some participants. Subjective and objective benefit has been reported. Participants benefit from group cohesiveness and empathy that decrease the sense of isolation caused by the disorder. Stress is reduced by a perceived increase in social support. Patients with AIDS, breast cancer, chronic back pain, and diabetes mellitus have enjoyed documented improvement in their physical or mental health. Patients may feel empowered through online groups. Added benefits accrue when CMC support is combined with FTF groups.

There are significant risks to online group participation, including delayed interactions, misinformation, arguments, loss of privacy, online stalking, and deception. Patients with suicidality, refusal to get better, or anxiety, psychotic, borderline, factitious, and obsessive-compulsive disorders may not be appropriate for online groups. Dishonesty is more likely in MUDs, newsgroups and chat rooms. Group members sometimes resent the heavy time and e-mail burden of online groups. Many are frustrated by the lack of physical contact with other members. Adverse psychological effects may ensue from online group use, such as Internet addiction, depression, social withdrawal, and loneliness. Recent work suggests that extroverts tend to use the Internet to extend their social networks, while people with deficient real-world social networks, and teens, may become more isolated. Knowledge of group psychodynamics, and specific training in online therapeutic techniques, may be invaluable for online group facilitators. While not well studied, the behavior of informal leaders online appears similar to that of trained facilitators.

Moderators oversee membership and technical matters, monitor appropriateness of posts, correct misinformation, facilitate therapeutic process, and mediate disputes. Many directories list existing groups, and instructions are available for those who wish to start new communities. Clinicians and website operators are often approached via the Internet for advice, and their practices in responding to these unsolicited requests vary. Liability and ethical concerns exist for clinical and mental health professionals who respond to queries, sponsor websites or moderate online groups. Many research methods have been used to study online groups. Experimental trials have assessed health or psychological outcomes from group use. CMC support has been experimentally compared to FTF support. Through covert observation, factors such as user demographics, number of members joining or leaving, time of use, message analysis, and social network analysis have been analyzed without disrupting naturalistic discourse by disclosing the investigator?s presence. Guidelines concerning ethics, confidentiality, and methodology have been developed for researchers who wish to study online groups.

Conclusions: Internet health support groups offer participants many benefits, with accompanying risks. To preserve privacy and enhance empathy, the author recommends closed, private, moderated listserv groups. Website developers and group moderators must consider user demographics, ease of use, confidentiality, group therapeutic process, and the balance of participant benefit and researcher needs when constructing and maintaining Internet support communities. 


\title{
Web Service of Aid to the Prostate Cancer Diagnosis using Expert System
}

\author{
Marcos A. Pereira \\ Institute of Biomedical Engineering, Florianopolis, Brazil
}

Abstract: Web services, as the name implies, are services offered via the Web. In spite of the Web services and consumers of Web services are typically businesses, making Web services predominantly business-to-business (B-to-B) transactions [7], its use can also be applied for other purposes besides the businesses world, like services of information for support to the decision, search and change of information, and data processing in expert systems.

The utilization of Web Service architecture for a system of the aid to information for support to the decision in medical area allows that several doctors can use the system in a simultaneous and independent way, making possible the concentration of clinical cases in an only database. However integrity and security factors are extremely necessary to the guarantee and secret of clinical data. The concentration of several clinical cases in an only database and of constant updating allow a contribution study about the behavior of the disease in the most different cases, and the search of means of better efficiency for its treatment.

Conclusions: Although the system is in development phase, this is a great tool of aid to the doctor, due to possibility of controlling all the variables involved in the process of the patient's accompaniment before his prognostic and also to provide him for middle of those factors and of the expert system the tendency of which prostate disease the patient possesses. The distributed characteristic together with the centralization of the data of several clinical cases, allow a great scientific study of the disease by multicentric way and constantly updated.

\section{[55]}

\section{Use of the Internet in practice of children's field hospital in a war region}

\author{
Vladinir I. Petlakh ${ }^{\mathrm{a}}$, Boris A. Kobrinsky ${ }^{\mathrm{b}}$ and Vladimir M. Rozinov ${ }^{\mathrm{b}}$ \\ ${ }^{a}$ Russian Centre of Disaster Medicine Protection, Russia \\ ${ }^{\mathrm{b}}$ Russia Scientific Research Institute of Paediatrics and Child Surgery, Moscow, Russia
}

Introduction: The public system of medical services in the Chechen Republic was mostly destroyed after 1995 and its main purpose has been to provide first medical aid to the population. Children suffered most of all, and as specialists left the region, the children's hospitals and clinics did not function due to battle actions and lack of financing. Therefore, a first childrens field hospital (CFH) was founded by the Russian Centre of Disaster Medicine Zaschita in the Gudermes Region of the Chechen Republic in April 2001. For the purpose of efficient operation of the CFH a telemedical bridge was organized and special equipment including a satellite system of the combined access "Helios Net" was installed. This system provided the most economically advantageous asynchronous exchange of information.

The equipment included: 1 . The satellite terminal, allowing for transfer of the information with speeds up to $64 \mathrm{~Kb} / \mathrm{s}$ through "Siren" - system and to receive the information with speed up to $1.0 \mathrm{~Kb} / \mathrm{s}$. 2 . A workstation for preparation of the medical documents. 3. Peripheral devices - digital video camera, digital camera, scanner. The consultations were organized as exchange of the medical documents on e-mail, and as videoconference, providing audio-video contact in real time, based on multimedia technologies. 
Results: 64 telemedical consultations were performed for 54 patients over 3 months. A network was created which included 4 telemedical centers, 6 scientific institutes and 5 large hospitals. It was especially important to maintain communication with medical centers of the Northern Caucasus. Most of the consultations was carried out through the Institute of Paediatrics and Child Surgery (50), through the Institute of biological sciences Northern Osetya (6), through Stavropol regional Hospital (5), and through other centers (3). 8 patients were evacuated directly for further treatment after telemedical consultations (4 to Moscow, 4 to clinics in the Northern Caucasus), 30 patients were sent for treatment to Hospitals in the Republic and to central hospitals, 7 for diagnostics, treatment tactics were specified in CFH for 9 children. Analyzing the first practical experience of realization of regular telemedical consultation in conditions CFH we should point out the high efficiency of telemedicine not only for diagnostics, definition of medical tactics, but also for operative decision of the issues connected with hospitalization of the patients to the specialized departments.

Conclusion: The satellite system and Internet were used in conditions of military conflict and in the absence of telephone communication, allow for regular communication with the Management Centers, to contribute to efficient decision-making in patient evacuation issues, and to arrange telemedical consultations. We can now recommend regular telemedical technologies in extreme situations for the settlement of clinical and organizational issues that will provide for significant improvement in the level of specialized medical aid.

\section{[56]}

\section{A virtual patient record implementation for emergency medical services}

\section{Mikaela Poulymenopoulou and George Vassilacopoulos Universtiy of Piraeus, Athens, Greece}

Emergency healthcare delivery involves the pre-hospital and the in-hospital emergency care. The prehospital emergency care is provided by the ambulance services staff at the place of incident and en-route and the in-hospital emergency care is provided by the hospitals staff at the emergency department of the hospital. In emergency healthcare delivery, from the time of a call to the ambulance service till the time of patients disposal from the emergency department of a hospital a variety of interrelated activities (administrative, paramedical and medical) are performed. During the execution of these activities medical staff of both the ambulance service and the emergency department of the hospital often require to have access to the patients past medical data in order to improve the effectiveness and the efficiency of medical decision making and to avoid redundant performance of medical procedures.

Virtual patient records (VPR) provide a means for integrated access to patient information that may be scattered around different healthcare settings that use heterogeneous, in general, systems to support their internal functions. VPRs promise to form the basis for creating virtual healthcare workgroups organized around patient care without regard on existing organizational boundaries. In emergency healthcare delivery, in particular, a specific portion of patient medical data is needed that is actually a view of the complete VPR. Thus, for the development of a VPR the appropriate technological infrastructure is needed for transforming the medical data produced and stored in different healthcare providers databases into a common format, for exchanging this data between healthcare providers and for retrieving relevant patients medical data according to users posed criteria. Clinical Document Architecture (CDA) is an ANSI approved document architecture for exchange of clinical documents using XML.

CDA standardize the markup of the structure and semantics needed for exchange of clinical documents supporting interoperability. Simple Object Access Protocol (SOAP) is the proposed standard for XML 
messaging, specifying a message envelope format and a method for data serialization using the HTTP, SMTP or other protocol. Web services provide a standard means of interoperating between different software applications, running on a variety of platforms and/or frameworks. Web services allow developing applications on top of existing information systems that are posted on the net for use by any authorized user. Thus, CDA can be used for defining and structuring the clinical documents that form the virtual patient record of a patient, SOAP can be used for exchanging clinical documents between the participating organizations and web services can be used for extracting medical data particular for emergency healthcare according to users posed criteria in the form of XML documents encoded to CDA format. The VPR for emergency healthcare was confined within the boundaries of a health district irrespective of the information systems used by the ambulance services and the district hospitals. In our implementation, three organizations are involved the ambulance service, a districts hospital and the District General Hospital (DGH).

Upon ambulance arrival at the emergency department of the hospital the medical data produced during the pre-hospital emergency care is transformed to CDA encoded clinical documents and is send through SOAP messaging to the hospital. After the patient exit from the emergency department of the hospital the medical data produced during in-hospital emergency care and stored in the hospitals database, is transformed to CDA encoded clinical documents. Then, both the clinical documents produced at the emergency department and the clinical documents received from the ambulance service are sent through SOAP messaging to the medical data repository of the DGH. In addition, during emergency healthcare delivery a web service can be executed from both the ambulance services and the emergency departments staff for retrieving relevant patient medical data for emergency healthcare from the DGHs medical repository according to users posed criteria and access rights. The use of web services and CDA for the implementation of a virtual patient record for emergency healthcare is presented that has been experimentally implemented to provide an automated framework for supporting an emergency healthcare process involving the Athens Ambulance Service (AAS) and the Emergency Department of the Genimatas District General Hospital of Athens (GDGHA). The virtual patient record supports emergency cases served by ground ambulance vehicles making available past medical data of the emergency patient to medical staff of both the AAS and the GDGHA.

\section{[57]}

\section{Design and Implementation of a Real Concept-Based EMR for the Heart Failure Patient Manage- ment}

Barbara Purin, Claudio Eccher, Paolo Giardini, Manuela Rigo and Stefano Forti Italy ITC-irst, Centre for Scientific and Technology Research, Trento, Italy

Abstract: The Electronic Medical Record (EMR) is the essential component of an integrated computerbased medical setting since it is used to store and manage patient health information. The purpose of this paper is to present a real application of a concept-based EMR for the heart failure patient management. The role of ontologies to allow a more effective data and knowledge sharing as well as to model and manage clinical information in a flexible and powerful way is widely recognized. Our approach is based on openEHR ontologies that allow us to build an interoperable, future-proof clinical information system. This work is part of the e-Heart Failure project funded by the Autonomous Province of Trento and started in June 2002.

Objectives: Health domain is huge and open-ended because of the constant evolution of the clinical information available from heterogeneous resources. Health information systems would have to be able 
to evolve over time according to the clinical knowledge changes. For these reasons Medicine has become one of the most active research area as regards the development of knowledge bases. The Electronic Medical Record (EMR) is the main component of an health information environment but it could have a too rigid structure if it is built ad hoc. Moreover, in order to enable reusing of existing knowledge bases and allow information sharing, especially over the web, we need knowledge integration methodologies and techniques so that complex and detailed concepts could be efficiently communicated. We try to approach these problems using ontologies.

\section{[58]}

\section{Digital-divide in eHealth applications in the city of Chicago}

Arkalgud Ramaprasad, Shobhna Gupta, Sridhar Papagari, Amit Prakash and Ramachandran Venkatasubramanian

University of Illinois Chicago, Chicago, USA

Objectives: Asthma bronchiale is the most common chronic disease in German children and adolescents and has a prevalence of over 10\%. The conventional structure of Asthma education, by consensus, follows Arbeitsgemeinschaft Asthmaschulung im Kindes- und Jugendalter and has proven to be successful for improving health. The study was to show that if the tailor-made Internet education tool, Forum-Telemedicine (FTM), was used as a follow-up for six months, patients would show a continued improvement of the management of their condition through patient education and empowerment. This would result in a reduction in cost of medication, less visits to GP / Hospital with acute symptoms and less time off work / school. FTM is based on the traditional education given to the patient when they are initially diagnosed but delivers an additional follow-up in a modern and new way. Its internet-platform contains an adventure game with asthma-related situations, a medical area with online peak-flow-monitoring and therapy plans, online chats with asthma-experts, an interactive quiz and a community area. It is not intended to replace the traditional education by an internet web page but to be used as a virtual patient lead continuation of the learning process.

Methods: An acceptance study with 50 patients as well as a prospective cost-benefit analysis alongside a non-randomised trial with 438 patients in 34 study centres with two intervention groups and one control group were conducted. Within the acceptance study patients participated in FTM following the traditional educational program whereas within the cost-benefit analysis both intervention groups received traditional training, but only one group the FTM-follow up. We collected data at the first visit as well as after six and twelve months.

Results: The results underline that this specific internet-based asthma education tool is widely accepted by patients resulting in a favourable benefit-cost ratio.

Conclusions: FTM as an add-on to traditional asthma education is an adequate channel for efforts to improve asthma self-management on a long-term basis reducing costs at the same time.

\section{[59]}

\section{Complementary and alternative medicine and the Internet}

Katja Schmidt and Edzard Ernst

UK Peninsula Medical School, Exeter, UK

Objectives: Our objectives were to assess the value (or otherwise) of the Internet in guiding the public regarding complementary and alternative medicine (CAM). Study design: 9 independent Internet/E-mail 
surveys Methods: We identified the main websites that patients with various conditions were likely to consult and evaluated these sites according to the following criteria: advice regarding conventional therapies, number and nature of CAM recommended, advice on disease prevention, and an overall judgement by the present authors regarding the safety of the advice provided. In total we assessed 83 web sites.

We also carried out 5 surveys assessing email advice given by CAM practitioners to a) a fictitious patient or b) a researcher. Results: Our results showed that on CAM for cancer websites a plethora of unproven CAM treatments is recommended with little consensus between different sites. Some websites had the potential to harm patients through misinformation or discouragement of conventional therapies. We found 12 websites overtly discouraging patients from using conventional treatments and 21 websites were graded as unsafe or possibly unsafe. Our results from the email communication surveys suggested that some CAM providers advise their clients irresponsibly; for instance, against government policy regarding the measles, mumps and rubella vaccination. Conclusions: In conclusion, the Internet is an important source for information on CAM. For the consumer there may be risks in using it for that purpose.

[60]

\section{The Role of the Internet in Global Continuing Medical Education/Continuing Professional Devel- opment: A Review of the Past and a Look at the Future}

\section{Lawrence Sherman \\ Jobson Education Group/Postgraduate Institute for Medicine, Melville, USA}

The need for continuous professional education by healthcare professionals is clearly documented. Lifelong learning is therefore an essential component in the career path of these people, most notably physicians. In the United States, most physicians need to participate in continuing medical education (CME) activities in order to maintain board qualification and thus be able to practice in their chosen specialty. Traditionally, CME has been offered through live events such as presentation at specialty society or association conferences, grand rounds programs, and individual meetings and teleconferences. Additionally, CME credits were available through the use of enduring materials such as written monographs, audiocassettes and videotapes. Recently newer media such as CD-ROMs and the Internet have emerged as delivery vectors for CME. This presentation will focus on the emergence of the Internet as an ideal method for delivering CME, often referred to as electronic CME or eCME, as well as examples of how this is being done. The Internet, by its nature and design, is a truly dynamic and cost effective method for disseminating information and education to a target audience when compared with traditional methods of delivery. Furthermore, the organic and dynamic nature of the Internet lends itself to both synchronous and asynchronous coursework, increasing the educational options available to the learner. The history of medical education on the Internet is similar to the availability of everything else on the web! In my estimation we are in the fourth generation of materials and information that is available. Initially online eCME was restricted to text-only documents. As anyone who has every tried to read a long text document on the web can attest, this can be difficult and at times painful. It was, however, an initial attempt at using what was then a new medium. As comfort levels with the Internet increased, the materials available be came slightly more involved, and had text along with some graphics. Instructional designers emerged as the most appropriate writers of web-based content, as they took into account the properties of the Internet when developing educational coursework. Most notably, text was written in ways that were supported by the size of the screen, and slides emerged as a better method for delivering 
text the bullet point replaced the full sentence! The third generation emerged when audio was added to text and graphics, and the learners were provided with more opportunity for true multimedia activities. At this time, however, the limitations of low bandwidth connections became evident. Learners with very slow connections were unable to hear the audio, or could only hear portions of it. Video, while available only in very limited amounts, was certainly not supported by these slower connections. Learners had to become more technologically savvy, and the need to download software that was needed to hear the audio was often a limiting factor for participation. At the time, many computers were not pre-loaded with multimedia software as they are today. Slow connections often prohibited even the most eager learner from participating in the online educational activities. Online CME providers needed to constantly monitor the average hardware profiles of their target audience members, and use this information to develop courses that were appropriate for the majority of their constituents. The third generation ended and the fourth began at about the time we slipped into the new millennium. While there is not scientific correlation between these two events, the temporal relationship is quite interesting! It was at this time that the average eLearner in the United States had a computer with a fast (Pentium II or above) processor, and a 56K bps connection to the Internet. Faster connections such as cable modems were becoming widely available for home use, offering connections at up to 100 times faster than a $56 \mathrm{~K}$ modem. Home computers were becoming increasingly more sophisticated, and multimedia audio and video software was often packaged with the computer, along with word processing, spreadsheet, and presentation software. Online CME began to take advantage of the faster connections and ubiquitous multimedia software along with the true dynamic nature of the Internet. Archived activities such as audio slide shows became omnipresent. Live and archived webcasts from national and international conferences became available. Megasites such as Medscape and Physicians Online offered information and education to an astonishing number of online physicians tens and even hundreds of thousands of American physicians were accessing these sites, and were using them for things as simple as email, and as sophisticated as webcasts and interactive educational activities. That brings us to what is available today. A search of the Internet for online CME activities will yield search results in the hundreds. All sectors of the education provider community are represented, with quality education being offered by universities, specialty boards and societies, and private organizations. Physicians in need of CME credits for relicensure or board recertification are able to find enough credit hours online to fulfill their requirements. The types of activities that are available vary and are truly multimedia in design. Live teleconferences are supplemented by web-based slides and graphics; for those unable to dial into these teleconferences, the audio and slides are available in an archived format for use at any time by anyone. Key presentations from conferences are almost routinely webcast, either in audio or video format. Like the teleconferences, these webcasts are typically archived for use by others later. These are two examples of the dynamic nature of the web, and of the cost-effective nature of using it. Web eCME also uses formats that the physician-learners find comfortable and are used to. Clinical medicine is taught using a case-based approach, and is segmented by specialty and disease. Online educational activities that are in these formats can now be found. There are virtual hospitals, virtual clinics, and even virtual tumor boards. Web sites that are devoted exclusively to case-based teaching have emerged. There is no shortage of eCME! This does, however, raise the question: Are physicians using the Internet? Those involved in eCME have heard this question for as long as eCME has been available. The answer to the question today, is far different from what it was in the past, and the numbers continue to grow. For example, a 1999 survey conducted by the American Medical Association demonstrated that only $37 \%$ of the respondents were active users of the Internet. Less than two years later a similar survey revealed that $70 \%$ of the respondents were active Internet users. Similar studies by the Royal College of Medicine in the United Kingdom, and by Harris Consulting demonstrated similar 
findings in broader survey populations. The target audience members are clearly available, and most are willing to participate in cCME. Between 60 and 95 percent of the respondents of these surveys indicated their willingness to participate in eCME. Another interesting fact is that the average retention rate for an instructor-led class is only 58\%. Data show that more intensive e-learning experiences enhance the retention rate by 25 to 60 percent. The next logical question then is: If we build it will they come? The answer to that is not as simple! Web site usage data indicate that physicians will only come to a web site if they know that it exists. Hoping that they find it by chance is not a smart approach. Although many physicians are sophisticated researchers and would be able to find appropriate sites by searching the Internet, they are confined by many variables, most notably time. Some of the megasites have down an excellent job of building name recognition and have loyal followings, however the CME activities on their sites are often difficult to navigate to. They are often in violation of the 3-click rule and lose many potential learners because of this. Other sites are designed and developed appropriately, but do nothing to market the sites to the target audiences, therefore only a small percentage of the target audience eventually participate in the educational activities. The sites that seem to be the stickiest and develop good repeat traffic are those that cater to a small, specific section of the target audience by specializing in specific conditions, therapeutic areas, or specialties. They offer easy to find educational activities as well as other information, links, and services that are pertinent to the well-defined target audiences. They also offer frequent updates, education in various multimedia formats, and activities that can be selected based on the learners connection speeds. The majority of these sites are in the United States and Canada, but slowly there are sites emerging from the United Kingdom and Continental Europe. Interestingly there is a great deal of international traffic on the US sites, even though most are not marketed internationally. This demonstrates the willingness of the international audience to participate in eCME, and the need for true international sites to be developed. Finally, what might be the most important difference between eCME and traditional CME is the interactivity. Whether synchronous or asynchronous the educational provider has the opportunity to receive feedback from the learners. This is a valuable tool, and helps to make the education better and serves as a needs assessment for future educational activities. The Internet also permits the use of sophisticated analysis of who is participating in the education, how long they are spending on the site and within each activity, and, when using a post-test, what learning is taking place. Follow-up impact studies can measure the ongoing impact of the education and are conducted easily via email. In summary, eCME has become an ideal method for delivering education on a global scale. Over the course of the last five years content and sites have become available that take advantage of learner preferences, and, when designed appropriately, provide the learner with education in a convenient and comfortable format. Metrics are used to determine appropriate use, and interactivity promotes better learning. Online CME will continue to grow and will become the most prevalent form of CME available to physicians worldwide.

[61]

Using a content management framework to implement a distance learning platform: The JALON project (Join And Learn On Network)

Pascal Staccini ${ }^{\mathrm{a}}$, Jean-Charles Dufour ${ }^{\mathrm{b}}$, Hervé Raps ${ }^{\mathrm{a}}$, Michel Joubert ${ }^{\mathrm{b}}$, Jean-François Quaranta ${ }^{\mathrm{c}}$ and Marius Fieschi ${ }^{\mathrm{b}}$

${ }^{a}$ Département STIC-NTIC, UFR Médecine, Université Nice-Sophia Antipolis, Nice, France

${ }^{\mathrm{b}}$ LERTIM, UFR Médecine, Université de la Méditerranée, Marseille, France

${ }^{\mathrm{c}}$ IUP ISANI, Université Nice-Sophia Antipolis, Nice, France 
Distance learning has become a strong subject of interest. Since year 2000, the French Ministry of Education and Research has carried out numerous calls for projects to encourage universities and teachers to publish academic resources and pedagogical material on the web. The "ESSQU@D" project ("Enseignement Santé, Sécurité et Qualité A Distance") submitted by the Information Technology Department of the School of Medicine of Nice was accepted after the first call. ESSQU@D offers a post-graduate diploma in the area of quality improvement and risk management in health care services.

During the first semester, our team concentrated on problems that may occur when using distance learning technologies. Three major problems were pointed out: 1) students, as well as professionals, do not know much about distance learning. Statistics point out that $25 \%$ of students give up the distance curriculum; 2) trainers for the diploma lack formal pedagogical knowledge because most of them come from the business world; 3) the methodological standards are recent and incomplete, web data-processing and publishing techniques are evolving rapidly. Finally, the concepts of distance learning platform and distance teaching can be interpreted in many different ways.

According to this analysis, strategic choices were made in order to minimize the risks. The second stage of the project thus consisted of: 1) analyzing and offering a tutoring model; 2) integrating pedagogical principles and teaching engineering in the development of the platform, in order to have a constructivist approach and provide users with collaborative tools; 3) analyzing and implementing techniques of acquiring and publishing course contents, while using open source standards.

Concerning the learning environment model, we defined the typology of pedagogical resources, their data model and their logic of use. We implemented the authoring/publishing platform (called JALON for Join And Learn On Network) within an object-oriented programming environment (ZOPE platform for $\mathrm{Z}$ Object Publishing Environment) including a workflow management process (content management product named PLONE).

Authors and students share the same space of knowledge, the first ones with an "add-modify-suppress" profile, the second ones with a "view" profile. The schema of navigation within a lesson proposes a list of interactive "sequences" and "quizzes". Four libraries of teaching components were made up: a library of electronic documents of various formats (doc, ppt, pdf, ps, swf), a video library containing movies of the original courses (Real ${ }^{\mathrm{TM}}$ format) and "rich media" courses (SMILE standard), a dictionary of terms or expressions, and a library of questions. Authors are able, by their own, to create numeric lessons by combining several components picked-up from each library through an interface that fits the learning environment model. The description of lessons uses the Dublin Core standard (built-in feature of PLONE). Besides, a stand-alone tool allows the creation of interactive questions (simple/multiple choice questions, questions using clicking or "drag and drop" areas); questions are imported into the platform using XML grammar. A specific module has been implemented to manage the content of curricula. A curriculum is structured as a tree which contains units composed of courses. Within our platform, the structure of curriculum is stored in one SQL table and presented as a tree-view control to the manager. After logon, the student has direct access to the courses he is allowed to read, according to the content of his/her curriculum. A student can declare that he/she read a course. His/her actions are traced in his personal folder. An author/teacher has access to the courses he builds. For each author, the platform clearly identifies courses in preparation and courses already published (the history of the status of the documents is managed by the workflow). For each course, an online discussion module can be added. Calendar, events, and news management tools are also available through the built-in features of the PLONE product.

The web site can be reached at: http://www.essquad.org (user: 'Etudiant', password: 'Etudiant'). Technical parts have to be improved to provide students and teachers with further user-friendly collaborative tools. A first evaluation of the use of this platform by students and teachers will be included in a national process of quality evaluation of the distance learning program that will start in September 2003. 


\title{
Internet resources and problems of pediatric oncology
}

\author{
Grigory Tseitlin $^{\mathrm{a}}$, Elena Ouspenskaia ${ }^{\mathrm{b}}$ and Marina Bialik ${ }^{\mathrm{a}}$ \\ ${ }^{a}$ Institute for Pediatric Oncology and Hematology, Russia \\ ${ }^{\mathrm{b}}$ Moscow Cancer Relief Society, Moscow, Russia
}

Oncology diseases are a very hard issue for plenty of people. In Russia it's even more complicated because of the absence of adequate resources of information, spreading of wrong and outworn data. And that is the reason why parents of the children with cancer are getting involved in very traumatic situation. Very often the parents don't receive from doctors full information concerning prognosis, different methods of treatment and possible complications. The information vacuum is usually filled in by false data. And as a result in some cases the parents refuse to get treatment, though modern pediatric oncology enable to cure more then $60 \%$ of ill children, and in cases of such cancer types as Hodgkin's disease, nephroblastoma, retinoblastoma $-90 \%$ of children survive.

Unfortunately, in Russia, especially in regions general pediatricians and other specialists are not always aware of pediatric oncology problems, and therefore may disorient the parents concerning prognosis and expediency of treatment. Information deficiency exists not only in medical sphere but also in humanitarian one, particularly in fields of psychological and social support of ill children and their families. Thus stressed parents don't know where they can get help. Our Internet project "Cancer Children Consultation" (CCC) is based on all considerations that we've mentioned above.

Target groups: parents of cancer children; people who have gotten threw cancer in childhood; different medical specialists; psychologists, educators, social workers; members of non-governmental organizations; students. As a matter of fact problems of pediatric oncology are not represented In Russian Internet. There are only a few sites of parent's organizations that discuss particular problems of cancer children. CCC was created by professionals in 2000 under financial support of "Project Harmony". The goal of the project was to provide relevant information on all the aspects connected with pediatric oncology.

Our site includes e-manual, different materials and documents concerning pediatric oncology. Team of qualified specialists (pediatric oncologist and hematologist, palliative treatment specialists, psychologist, geneticist, layer, social worker) provides permanent service of answering on-line questions. Main divisions of the site: "Our consultants", "Questions", "Manual", "Library", "Parent's stories", "Useful links". E-Manual includes: "Organization of pediatric oncology service", "Genetics", "Solid tumors", "Brain tumors", "Hemoblastoses", "Psychology", "Palliative care", "Hospice", "Social and juridical aspects". Thus our manual contents all the problems of pediatric oncology. CCC attracts a lot of attention. It was highly estimated by parent's organizations and professionals.

In conclusion, there was created Internet resource providing information support to one of the most undefended groups of population. It also enables further development in this field. At the present we prepare new materials for CCC. We suppose that CCC helps to resolve the problem of connecting cancer children and their families with professionals.

\section{[63]}

Roles in the domain of electronic health records: Security, ownership and access management

Frank Kurt Uckert ${ }^{\mathrm{a}}$, Sven Tesmann ${ }^{\mathrm{b}}$, Hiep Doan ${ }^{\mathrm{c}}$ and Hans-Ulrich Prokosch ${ }^{\mathrm{d}}$

${ }^{a}$ Institute for Medical Informatics and Biomathematics, University Hospital Munster, Germany 
${ }^{\mathrm{b}}$ Institute for Medical Informatics and Biomathematics, Germany

${ }^{\mathrm{c}}$ Children's University Hospital, Germany

${ }^{\mathrm{d}}$ Germany Institute for Medical Informatics, University of Erlangen-Nurnberg, Munster, Germany

Objectives: Decisions and navigation through medical information needs cooperation between patients and physicians as partners. On the one hand the physician has the professional knowledge and on the other hand the patient is the only common and central key figure in the system, where several physicians or other HCPs are working together. This cooperation is slowly transformed into a network of care [1]. While several projects build up electronic patient records within linked units of hospitals, clinics and practices, other projects try to implement electronic health records (EHRs) for the patient himself, the integration between these projects is still a problem. The EHR akteonline [2] offers a basic platform for information and communication among HCPs and even including the patient. The patient is able to document various data into his EHR, where it can be viewed, downloaded and/or complemented by HCPs, who therefore can use the EHR as a medium for communication. With standardized data exchange interfaces the contents can even be exchanged automatically. The objective of this project was to construct a role based access management for an EHR with special views on a patients medical data for HCPs. The system shall be usable over the internet and has to work by only using a ubiquitous web client.

Methods: As an underlying system the EHR akteonline, a collection of medical data of one patient, was used. It is accessible via the internet and contains non-professional information like wellness information, diets or information on physiotherapy as well as medical data from clinical episodes. The in- and output of data uses modern web technology or data interchange between different information systems. Data exchange can be handled via XML based on the Clinical Document architecture CDA [3], and even DICOM [4] images can be imported and viewed by the patient. As additional services educational information for patients as well as for physicians is included. Time triggered messages can remind the patient of events like vaccinations or appointments. Technical data safety via hard- and software is as well considered as the data storage. Internal structures enable the patient to handle a complex access management system with specific authorization levels for different persons [5], which was a good foundation for developing a role based access management. The roles for an access management for an EHR were found to be: - the user - deputies - persons with predefined one-time access - HCPs with continuous access and changing rights The role based model, realized within the last year, shall be illustrated by an actual clinical scenario taken from the pilot project at the childrens university hospital of Munster.

Results: In the waiting room of the ward the parents of the six year old patient set up three EHRs, one for each of them. The parents make themselves deputies of the child, so they have full access to the childs EHR and the system is able to log and sign data changes correctly. During working with the system, a parent can choose between his own record and the one he/she is deputy of. Two continuous HCPs, the general practitioner (gp) at home and the hospital specialist, are already present in the system and so the deputy just adds to the patients EHR the corresponding address book entries and assigns the freely defined roles gp and specialist to them. After granting access rights for reading and/or writing in some subfolders of the EHR, the specialist half-automatically adds lab result data and a referral letter (CDA). At home the deputy adds some medial data like the family anamnesis, personal and basic medical data, examinations and images to the patients record. He/she also reads some (automatically added) information from the university hospital about food, playing and financial aspects concerning the very rare and special disease. For a visit to another specialist, who is not attended frequently, the deputy prints 
out some transaction numbers (TANs), asks the hospital by phone to transfer two special $\mathrm{x}$-ray images (DICOM) and sets the access rights of the TANs for reading lab data and $\mathrm{x}$-ray images and adding objects like letters. The second specialist is now able to access the data and upload his findings via his usual web browser. This last example could also represent the way to question a HCP from another country over the internet. To give the HCP a tool to handle more than a few EHRs of patients, he has got a totally different view after login than a normal user has.

Conclusions: An access management for an EHR at least needs the described four different arts of access. Implementing all four into akteonline showed that with the role based model signing of uploaded data, managing data ownership and logging accesses is only now possible or much easier than before. Also the new HCPs view on several EHRs seems to be promising and has to be evaluated further.

[64]

\title{
Secure exchange of medical images and other objects within a heterogeneous network of experts with the largest possible client simplicity
}

\author{
Frank Kurt Uckert ${ }^{\mathrm{a}}$, Lars Brinkmann ${ }^{\mathrm{b}}$, Gunnar Fischer ${ }^{\mathrm{b}}$ and Michael Paulussen ${ }^{\mathrm{b}}$ \\ ${ }^{a}$ University Hospital Munster, Germany \\ ${ }^{\mathrm{b}}$ Childrens University Hospital for Oncology, Munster, Germany
}

Abstract: Patients with rare diseases are often treated in multicenter trials. The trial centers are spread even over national boarders. Mainly the fields of radiology and pathology account for a substantial workload by medical data (and especially image) transmission between clinics and trial centers via paper/film based postal service. The design of an object clearinghouse by using internet technologies promised to come up to prior investigated expectations. Special points of interest were handling of DICOM images over the network, security and cost considerations as well as the ease of use. Comments: Introduction German Pediatric oncology patients are treated within multicentric trials. The trial centers are spread all over Germany and other countries. Mailing, archiving and retrieval of forms, radiographic images and images of specimens account for a substantial workload at trial centers and participants. Telemedicine networks could provide digital copies of study material on demand to trial participants, eliminating delays from postal delivery and manual retrieval. Also external experts can be enabled to share common views on selected medical data. At the same time the clinical data can be accumulated for scientific and educational purposes in anonymous form. Shown by a study made in 2002, the expected potential of a secure and flexible exchange of medical images is seen as valuable by concerned medical experts [1].

Objectives: The Objective was to build a central clearinghouse fulfilling the following goals without giving access to a radiology system directly. The clearinghouse had to - be easy to use, - use existent technology, - be ready to implement new features easily, - be as independent from time and place as possible and reasonable, - work in heterogeneous environments of the medical and technical experts, - use a very simple client, - be cheap, - handle and convert DICOM images as well as other common image formats, - act as a DICOM network node to receive images directly from the imaging device, - give users the possibilities to easily handle the access management, - manage access rights in a role based model, - give one-time access to medical experts who are not a member of the corresponding trial, - sign the data, - give the administrator of a trial the tools to handle information flow and merge patients, which are entered by more than one institution and - use the data for scientific and educational purposes as well. 


\section{References}

[1] N. Graf, M. Paulussen, T. Huf, T. Ganslandt, J. Stahl and H. Jurgens, Telemedicine in pediatric oncology, Klin. Padiatr. 214 (2002), 8-19.

[2] B. Bergh, A. Schlaefke, M. Pietsch, I. Garcia and J. Vogl, Evaluation of a "no-cost" Internet technology-based system for teleradiology and Co-operative Work, Eur Radiol 13(2) (Feb 2003), 425-434.

[3] T.H. Le and N. Malhi, Thin client (web browser)-based collaboration for medical imaging and web-enabled data, $J$ Digit Imaging 15(Suppl 1) (2002), 261-263.

[4] Y. Kondo, Medical image transfer for emergency care utilizing internet and mobile phone, Nippon Hoshasen Gijutsu Gakkai Zasshi 58(10) (Oct 2002), 1393-1401.

[5] A. Palombo, T. Haigh, J. Ferguson and D. Pedley, Can paediatric radiographs be accurately interpreted using an interhospital telemedicine system? J Telemed Telecare 8(Suppl 2) (2002), 70-72.

[6] ImageMagick Studio LLC. ImageMagick. Internet: http://www.imagemagick.org/ (Last access 07.08.03, 10:00 CET).

[7] Kuratorium Offis e.V.. Dicom.Offis.De. Internet: http://dicom.offis.de/ (Last access 07.08.03, 10:00 CET).

[8] F. Uckert and M. Paulussen, Telemedicine Overview. Internet: http://imfl.uni-muenster.de/index.php?telemedizin (Last access 12.08.03, 15:00 CET).

[65]

A Latin America Telemedicine Social Perspective. From A Colombian Telemedicine Center Initiative

Jorge Alberto Velez

Colombian Telemedicine Centre, Cali, Colombia

Introduction: Latin American population is in the need for health assistance any time in any place. However, social, political and technological situation establishes a difficult spectrum. In one hand, fragmented and reforming health systems; on the other hand, growing poverty, social inequity and difficult access to new technologies. Nowadays, the application of information technologies in health, Internet and telemedicine show different advances in the different countries on the region. The most relevant thing is that there is not enough level on knowledge and equipment in telemedicine. The scarce of political will shows that in some countries telemedicine are considered as a tool for the future. Here, the big risk is to allow that the future surpasses us. The purpose of the Colombian Telemedicine Centre and the Internet Health and Medicine Association is to use the Internet as the platform and environment, which facilitates information and medical knowledge distribution. This establishes the basis to our focus of telemedicine and telehealth in wider areas. Telemedicine, particularly on Internet constitutes an easier, economic and reachable way to support un-served populations located in remote locations, which are distant from specialised centres and health professionals. This would be helpful on decision-making. In contrast, citizens must be empowered with their role in the health care attention process. In the case of Colombia, where this initiative was born, internal war and terrorism become not only a threat for national security, but also a potential risk for neighbourhood nations. For this reason strategies have been proposed to create a regional front against terrorism in this scenario. The Colombian Telemedicine Centre proposes a development model on telemedicine for Latin America through a social perspective in which mayor areas are framed. For example: - Support to displaced population. - Distance learning for landmine victims and health teams who attend them. - A telemedicine support model for national and regional security. - A tele-educational component. At the moment there are some advances that have been presented in international scenarios.

Methods: We consider the following methodological aspects: a. Software architecture with a set of modules and facilities: The seven modules that hade been detected up to now. The Devices module had 
to do with the hardware, and drivers to be used by the system in order to communicate directly with the user. The process starts when the user interacts with a Device object. This device has a graphical representation, which is read from the object module. The purpose of the interaction module is to make the systems hardware independent. It communicates the inputs from the user to a Session object in the Information module. Also, it displays the appropriate graphical user interface for the service, which can display graphic interaction elements such as a stereoscopic visualisation element, or educational aids like a video. Also, it transmits the output of the system to the hardware devices. In this module a session is started. We have detected that a Session object is represented by a trainees practice lesson. The Services module deals with the communication and functioning matters of the service. A service is started as part of a session. A Service object using a protocol sends requirements to the Mediation module and graphical user interface attributes to the Session module, which communicates them to the interaction module. The Mediation module handles the processing requirements from the Service module communicating with the Object module. It also frees modules Devices, Interaction, Services and Session from complex calculations. Mediation is achieved via some specific type of service like for example, a virtual simulation, which has a number of surgical steps with medical objects. Each type of service knows its own requirements. The required information is transmitted by the information module, according to the service in a standard format. Sessions and evaluations are the main source of information in the architecture. The Object module deals with organs and tools modelling. Here, The Behaviour sub- module manages the mathematical, physical and artificial intelligence processing for the virtual objects. Also, the status and representation of an object are the responsibility of the Geometry sub- module. An object can be represented for collision detection, for visualisation or for transmission. The Communication module, which is the distributed platform where the application runs, uses a Messenger object that receives or places the transmission representation of an object. $b$. Knowledge of the current assistance process and its improvement opportunities, through the following steps of the process: Mapping of the of conflict areas, the displaced populations and land mines areas seeded in Colombian territory, Localization of victim populations in each department, Access to the Colombian Telemedicine Center facility, an architectural milieu with clinical applications, distance learning and humanitarian assistance approach. c. Knowledge of the need of health care for the displaced population and landmines victims. Health care needs detection, Levels I and II from health care resources utilization, Identification of specialized medical resource needs in each area, Interaction of local health care team with virtual health care team from the Colombian Telemedicine Center and from the Center of International Rehabilitation, partner in the support of land mines victims, Displaced populations and Land mines victims Problem solving approach, Follow-up, Health care education. d. Further applications of Epidemiological georeferentiation, application of a Geographical Information System (GIS). For supporting the Homeland and regional security, we in conjunction with the leaders of the Latin American Telemedicine teams, present the future operative role of Telemedicine in humanitarian assistance. This is closely related with the international humanitarian rights matters and health care. This operative is proposed from the forecasting of the technological infrastructures and national plans on Telemedicine in the region. In order to reach our goal, we work according to the current governmental telecommunications and health policies to build a specific role of Telemedicine and related initiatives in the homeland security landscape. We see this as an element that can contribute in the development of citizen democracy in Latin American countries.

Results: a. Described initial results of a generic, extensible and modular software architecture and tele-services. b. Telematics attention model in each area: a. Support for Conflict areas; b. displaced population: c. Landmine victims support; d. distance learning support. c. Latin American research 
centres and teams network connected for the development of tele- education and distance learning with a virtual environment focus. Set of web based simulators in each clinic field; planning instrument; web expert decision support. d. The role of telemedicine in national and regional security issues. Discussion Latin American social, political and technological situation establishes several challenges; the Colombian Telemedicine Centre proposes a social perspective for telemedicine in the region from advances and application in the field. Our proposal considers the Internet platform as a feasible, economic and reliable environment where clinic and decision support applications must run. This can help health specialists to provide quality health care. The environment proposed by software architecture and other tele services permit the application of clinical techniques and medical services such as inter consultation, second opinions, medical information and imaging exchange, video-conferencing. Moreover, In the case of more developed cases it also permits the use of artificial intelligence and virtual reality where we count with prototypes, which have been presented in ATA 2001, MMVR 2002, ATA 2002, ICT 2002 y CARS 2003.

[66]

Diagnostic agreement and usability issues for a web-based proficiency testing system in cervical screening

Federico Viel ${ }^{\mathrm{a}}$, Vincenzo Della ${ }^{\mathrm{a}}$, Francesca Demichelis ${ }^{\mathrm{b}}$, Jonni Santi ${ }^{\mathrm{b}}$, Paolo Dalla Palma ${ }^{\mathrm{b}}$ and Carlo Alberto Beltrami ${ }^{\mathrm{a}}$

${ }^{\mathrm{a}}$ University of Udine, Italy

${ }^{a}$ City Hospital of Trento, Italy

Introduction: Quality control is crucial in cervical screening programmes. This can bring the form of integrated continuing education and proficiency testing initiatives for the personnel involved (cytotechnicians, biologists and pathologists). In 1996, the European Federation of Cytology Societies has established a Committee For Quality Assurance, Training And Education (QUATE), which developed a method for proficiency testing, according to which a subject should answer a written test, screen 10 full PAP smears in two hours and diagnose on 20 fixed microscope fields. As the glass slides should be the same for all the subjects, the test is usually carried out during congresses, by using 20 microscopes each of which hosting a specific glass slide; the subjects pass from one microscope to the other, in sequence. Their organisation details make it difficult to carry out many tests. A solution is given by digital slides (1), i.e., digital copies of glass slides. They can be copied and effectively distributed, differently from what happens with glass slides, which are fragile and, in case of cytology, definitely unique. This may allow for a more effective implementation of quality control programmes, either in cytology as well as in histology (2). Among the disadvantages, digital slides are really large, being composed by thousands of images. The present paper describes a preliminary trial on the use of digital slides for proficiency testing.

Methods: 6 PAP smear glass slides and 6 thin layer slides have been digitally acquired at the institute of Pathology, Udine; 4 per type were chosen for the trial, while the others were used for training. PAP smears have an area of $21 \times 45 \mathrm{~mm}$; thin layer samples are spread on a circle of $19 \mathrm{~mm}$ diameter. Due to the different physical sizes of the samples, the average number of images per digital slide varied between 6000 and 30000. For simplicity, the cases were then burnt on a DVD, together with a specific web-based viewer. The viewer was also able to send data about interface usage to a server on the Web (3). The viewer can be used with a recent Web browser, as it is written in HTML and Javascript. Six cytotechnicians of the City Hospital of Trento have been trained in advance to use the digital slide 
viewer, by means of a lecture illustrating the features of the software and of the trial scope, and by giving access to the training cases for two weeks. During the trial, the computers on the internal network of the Institute of Pathology of Trento have been used, and their features (processor, memory, monitor size) were registered. cases were available on a local server. The diagnoses given by the cytotechnicians have been gathered using the QUATE forms. At the end, each subject has been interviewed about the system features. The diagnoses were compared with those given on glass slides at the Institute of Pathology of Udine. The time needed for diagnosis was evaluated too.

Results: Diagnostic agreement was obtained in all but one case, for which a minor discordance has been found. The time needed to complete a diagnosis on a digital slide is much higher than on glass slide (20 minutes for thin layer slides, about 28 for PAP smears, versus 5-10 for a glass slide). This is partially due to the subjects habits, much more acquainted with microscopes than computers, partially on the network performance, and partially on the interface features (which will be modified after the experiment).

[67]

\section{Speech Acts, Documents, and Medical Phenomena: An Investigation in the Ontology of Organi- zations}

Lowell Vizenor $^{\mathrm{a}}$ and Barry Smith ${ }^{\mathrm{b}}$

${ }^{a}$ Institute for Formal Ontology and Medical Information Science, University of Leipzig, Germany

${ }^{\mathrm{b}}$ Department of Philosophy, University at Buffalo, NY, USA

Introduction: There are many efforts underway to develop efficient ways of sharing information across healthcare systems and organizations. One spearheading effort is Health Level 7 (HL7), whose stated mission is: "To provide standards for the exchange, management and integration of data that support clinical patient care and the management, delivery and evaluation of healthcare services" [1]. Stefanelli [2] has argued that since HCOs differ with respect to their structure, personnel, resources, etc., it is difficult to share guidelines across systems and organizations. Hence, he argues, guidelines must be combined with models of resources and processes of patient care that are based on a sound ontology of organizations. The present paper outlines the basis of such an organizational ontology, starting out from the ideas in speech act theory central to the HL7 Reference Information Model (RIM). In what follows we apply principles from the philosophical ontology underlying speech act theory to the HL7 RIM, and draw conclusions for an ontology designed to support efficient communication of medical information within and between healthcare organizations.

The Ontology of the HL7 RIM: The HL7 RIM [3] is a system for the standardized representation of clinical data based on an identification of the life cycle of events that messages or groups of related messages within and between healthcare organizations will carry.

Speech acts play a central role in the RIM, since its designers maintain that health care organizations are primarily constituted by "intentional actions, performed and recorded by responsible actors." An Act-Instance is "a record of something that is being done, has been done, can be done, or is intended to be done." No direct reference in the RIM is made to natural events such as a patient's heartbeat. The reason for this is that every Act-Instance must be an act that can be attributed to someone. Thus the natural event of a patient's heartbeat may be recorded as observed, but there is no record of the event itself, independent of the observation of, say, a physician. The relevant record-observation Acts are assigned a mood Code that distinguishes them from records of intended or ordered services. The latter 
will standardly progress from defined, through planned and ordered to executed, these successive stages being represented as the mood of the Act.

Following [4] the designers of RIM have emphasized the importance of understanding the medical record not as a collection of facts, but as "a faithful record of what clinicians have heard, seen, thought, and done." Acts as statements or speech acts are the only representation of real world facts or processes in the HL7 RIM. The truth about the real world is then constructed through a combination (and arbitration) of such attributed statements only, and there is no class in the RIM whose objects represent "objective states of affairs" or "real processes" independent of attributed statements. As such, there is no distinction between an activity and its documentation. Every Act includes both to varying degrees.

We agree that a healthcare organization can be viewed at one level as a complex of interrelated speech acts through which the actions of organizational agents are coordinated. However, we maintain that an adequate ontology of healthcare organizations should correspond to reality itself in a manner that maximizes descriptive adequacy within the constraints of formal rigor and computational rigor.

As a first step towards such an ontology we draw on the work of the two major founders of speech act theory, namely Austin [5] and Searle [6], to set forth the division between those provinces of the reality of healthcare organizations which are, and those which are not a part of physical reality but which exist because and to the extent that there are documents that record their existence. Within the context of a healthcare organization, there are documents that record, for example, the existence of an insurance claim, a request for a medical test, an obligation to perform a surgical procedure and so on. The associated claims, requests, and obligations then coincide with no part of physical reality but they serve to hold the organization together as a social object. Abstract entities such as these are brought into existence by the appropriate corresponding speech acts. They are truly such that, as the HL7 RIM might put it, 'there is no distinction between the entity and its documen tion'. For other sorts of entities, however, this is not the case.

Some social entities such as doctors and clinical wards coincide with physical objects or events and provide the scaffolding which supports those abstract entities that bind together an organization - entities which are not real, but which are yet tied to contexts of human behavior [7,8].

Attempts to develop standards and guidelines for interoperability between healthcare information services will be aided by an ontology of organizations that is maximally representative of organizational reality. The neglect of objective states of affairs and real processes by HL7 RIM - and more generally the neglect of the context within which messages are conveyed - places obstacles in the way of an adequate ontology of healthcare organizations of the sort which, as [2,9] show, is needed for effective knowledge management. We will show how an ontology can be developed along these lines which can provide an account of social organizations in terms of which efficiency of communication and knowledge management can be enhanced.

\section{References}

[1] http://www.hl7.org.

[2] M. Stefanelli, The socio-organizational age of artificial intelligence in medicine, Artificial Intelligence in Medicine 41 (2002), 36-43.

[3] Version: V 01-22 (2/22/2003) http://www.hl7.org.

[4] A.L. Rector, W.A. Nolan and S. Kay, Foundations for an electronic medical record, Methods Inf Med 30 (1991), 179-186.

[5] J.L. Austin, How to do Things with Words, Oxford: Oxford University Press, 1962.

[6] J.R. Searle, Speech Acts. An Essay in the Philosophy of Language, Cambridge: Cambridge University Press, 1969.

[7] B. Smith, John Searle: From Speech Acts to Social Reality, in: John Searle, B. Smith, ed., Cambridge: Cambridge University Press, 2003, pp. 1-33. 
[8] B. Smith and J.R. Searle, The Construction of Social Reality: An Exchange, American Journal Economics Sociology 62(2) (2003), 285-309, repr. in: Searle on the Institutions of Social Reality, L. Moss and D. Koepsell, eds, Oxford: Blackwell, 2003.

[9] A. Kumar, B. Smith, M. Stefanelli, S. Quaglini and M. Piazza, Implementing Clinical Guidelines in an Organizational Setup. IFOMIS Technical Report http://ontology.buffalo.edu/medo/Functions-Workflow.pdf.

[68]

\section{Swiss-Prot: Medical information on the web - how a protein knowledgebase can serve the medical community}

\section{Yum Lina Yip}

Swiss Institute of Bioinformatics, Geneva, Switzerland

The World Wide Web has made access to information straightforward and easy. Presently, a variety of databases and tools for medical information retrieval are available on the Web. The Swiss-Prot protein knowledgebase (http://www.expasy.org/sprot/) is a curated protein sequence database that connects amino acid sequences with current knowledge in the Life Sciences. It provides a high level of annotations, standardized nomenclature and direct links to other bio-molecular databases. Although Swiss-Prot is not a medical or disease-centred database, it stores a wealth of information of interest to the medical community. This medical annotation effort is embedded within the framework of the Human Proteomics Initiative (HPI), a major project that aims to annotate all known human protein sequences and their mammalian orthologs. Specifically, Swiss-Prot provides description of diseases and/or polymorphisms associated with protein alterations. Medical keywords are constantly being created to allow the users to retrieve information on proteins that are implicated in diseases. Information on proteins used as therapeutic drugs, as well as cross-references to the Online Mendelian Inheritance in Man database (OMIM), Ensembl, PubMed and other human gene databases (GeneCards, GeneLynx, Genew) and/or specialised mutation databases, are also provided. Currently (Release 41.23 of 9-Sept-2003), Swiss-Prot contains a total number of 9'690 annotated human protein sequences. Among these entries, 2'071 provide data on genetic diseases and/or polymorphisms. A total of 16' 716 variants have been entered, most of which are associated with disease state (up-to-date statistics are available at $\mathrm{http} / / / \mathrm{www}$.expasy.org/sprot/hpi/hpi_stat.html). As single amino acid polymorphism (SAP) is the type of mutation most frequently related to human diseases, new Swiss-Prot variant web pages are created to facilitate the users to obtain the most relevant information about each human SAP recorded in the database. The variant web pages further provide additional structural information on the variants. In particular, wherever possible, SAPs are mapped onto protein 3D-structure via a stringent homology modelling pipeline. The 3D homology models are automatically built and updated with each weekly Swiss-Prot release. It is anticipated that the structural analysis of protein variation will strongly complement the existent sequence information, and thus aid in the interpretation of SAP and disease relationship. The Swiss-Prot variant pages are accessible from the NiceProt view of a Swiss-Prot entry on the ExPASy server (http://www.expasy.org/), via a hyperlink created for the stable and unique identifier FTId of each human SAP. The integration of sequence data, genetic information, physiological, biochemical and structural data is essential for the understanding of biological processes underlying disease state and health. The Swiss-Prot protein knowledgebase provides the medical community with such integrated information. 
[69]

Integrating internet based technologies for international medical collaboration and clinical trials

Kenneth Paul Youngstein

WorldHealthCom, Zuerich, Switzerland

Abstract Clinical trials are more numerous, larger, longer, more complex, more international, more rigorous, more regulated, more outsourced, more expensive, and consequently more difficult to manage. Attempts to use internet based applications to facilitate clinical trials have focused largely on data collection, submission and analysis. Today, however, the majority of clinical trials are still paper based. Clinical trials require more than managing data; they require the management of people, processes and knowledge. This presentation examines the requirements for internet based applications for managing international clinical trials, including: understanding the clinical environment, understanding the cultural differences of the countries involved in the trial, organizational issues, recruitment, training, collaboration, document development, document management, monitoring performance, and communication over the course of the trial. It stresses the importance of integrating applications into one easily accessible, open and unified platform. 